\title{
Les productions lithiques de l’Archipel indonésien
}

\author{
Hubert Forestier ${ }^{1}$, Michel Grenet ${ }^{2}$, Antony Borel ${ }^{3}$, Vincenzo Celiberti ${ }^{4}$
}

1. Muséum national d’Histoire naturelle, UMR 7194 CNRS-MNHN-UPVD, Institut de Paléontologie Humaine, 1 rue René-Panhard, 75013 Paris, France. Email: hubforestier@gmail.com

2. UMR 5608 Traces, maison de la recherche, Université de Toulouse II - le Mirail, 5 allée Antonio Machado, FR31508 Toulouse, France. Email: archelmi@gmail.com

3. Muséum national d’Histoire naturelle, UMR 7194 CNRS-MNHN-UPVD, Musée de l’Homme, 17 place du Trocadéro, 75116 Paris, France. Email: borel@mnhn.fr

4. Université de Perpignan Via Domitia, UMR 7194 CNRS-MNHN-UPVD, Centre Européen de Recherches

Préhistoriques de Tautavel, Avenue Léon-Jean Grégory, 66720 Tautavel, France.

Email: vincenzo.celiberti@cerptautavel.com

\section{Résumé :}

Proposer une synthèse sur la préhistoire d'un archipel et de ses assemblages lithiques n'est pas chose facile, d'une part à cause de l'immensité de l'espace concerné mesurant 2 millions de $\mathrm{km}^{2}$, d'autre part du fait que la notion de Paléolithique y est difficilement applicable et notamment celles de «Paléolithique supérieur » ou d’Epipaléolithique-Mésolithique établies en Eurasie occidentale. L'Indonésie et ses myriades d'îles et îlots (environ 18000) s'inscrivent dans un rectangle $5000 \mathrm{~km}$ sur $2000 \mathrm{~km}$ de part et d'autre de l'équateur ce qui en fait le plus grand archipel du monde. Cette aire géographique immense s'étirant sur un espace maritime d'environ 6 millions de $\mathrm{km}^{2}$, ne nous permet pas aujourd'hui de traiter exhaustivement l'ensemble des groupes industriels, des faciès ou des cultures préhistoriques, c'est pour cela que nous aborderons les principaux. L'Indonésie occupe une place privilégiée pour l'histoire des hommes fossiles qui la rend incontournable dans les connaissances de la variabilité des comportements des hominidés en contexte intertropical. Cette contribution a pour objectif de dresser un bilan critique et objectif des différentes méthodes de taille rencontrées depuis 1 million d'années sur les principales îles à partir d'une sélection de sites dont la stratigraphie est bien établie et bien datée.

Rares sont les technocomplexes bien définis avant l'Holocène, période où l'insularité de cette aire géographique s'est fixée avec la remontée marine marquant progressivement le début de l'histoire des archipels insulindiens. Parmi ceux-ci nous citerons le « Toalien » à Sulawesi (faciès à pointes), le "Sampungien " (faciès à pointes) et les industries sur éclats de Song Keplek ou "Keplekien » (débitage orthogonal) dans l'Est de l'île de Java. Hormis ces trois traditions techniques qui restent individualisables sur un plan typo-technologique, il n'y a pas dans l'ensemble du matériel rencontré, de faciès différentiables associés à une dénomination d'outils spécifiques. En règle générale, les industries lithiques du Pléistocène supérieur et de l’Holocène indonésien répondent à une production basique d'éclats et d'outils sur éclats avec de multiples variantes régionales. Le mode de débitage est très largement à la pierre dure, non Levallois, rarement Discoïde, non lamino-lamellaire tel qu'on peut le rencontrer en Europe de l'Ouest ou au Proche et Moyen-Orient. Contrairement à l'Asie du Sud-Est continentale où il est encore plus difficile de discerner un Paléolithique ancien, moyen et récent du fait de la continuité d'industries toutes réalisées sur galet (Hoabinhien et autres), l'Indonésie qui devient

Published by the School of History, Classics and Archaeology, University of Edinburgh ISSN: 2055-0472. URL: http://journals.ed.ac.uk/lithicstudies/

This work is licensed under a Creative Commons Attribution 2.5 UK: Scotland License. 
insulaire à la marge du Pléistocène et de l'Holocène propose une hétérogénéité des assemblages lithiques sans précédent. C’est-à-dire une diversité dans les modalités de production lithique selon différentes chaînes opératoires de façonnage (galet, pointe de type de Sampung, biface...) ou de débitage (discoïde, orthogonal et laminaire).

Le but de cet article est donc de présenter de façon synthétique les principaux ensembles lithiques de l'Archipel indonésien à partir d'une sélection d'îles sur lesquelles des assemblages lithiques ont pu être correctement documentés. Nous nous intéresserons ainsi aux îles de Sumatra, de Java, de Kalimantan (Bornéo), de Sulawesi, et à quelques autres plus orientales qui, comme Timor et Flores, ont, ces dernières années, livré des découvertes de premier plan en paléoanthropologie et en préhistoire. Par commodité, nous avons été obligés de procéder à des coupures régionales et chronologiques (Pléistocène ancien-moyen et Pléistocène supérieur final-Holocène ancien) qui permettent d'exposer au mieux cette synthèse sur des assemblages lithiques qui n'ont pas tous fait l'objet d'études technologiques approfondies au sens où nous pouvons l'entendre en Europe avec l'utilisation du concept de chaîne opératoire.

Mots-clés : Indonésie; Asie du Sud-Est insulaire; Discoïde; débitage orthogonal; débitage laminaire; pointe bifaciale

\section{Introduction à la mosaïque des industries}

L’Indonésie est une région insulaire privilégiée d'Asie du Sud-Est pour comprendre la dispersion, l'isolat, l'extinction et les comportements techniques des premiers homininés. La découverte d'homininés très anciens hors d'Europe dès la fin du XIX ${ }^{\text {ème }}$ siècle a favorisé la recherche préhistorique en Asie et notamment en Indonésie où E. Dubois en 1891 a mis au jour les restes du premier Homo erectus connu (« Pithécanthrope ») sur les rives de la rivière Solo à Java Central. Avant de connaître des enjeux propres à la science préhistorique et aux outillages de pierre taillée, l’Indonésie resta longtemps un des pôles fossilifères les plus convoités et compte jusqu'à aujourd'hui pas moins de quatre espèces humaines: Homo erectus, Homo sapiens, Homo soloensis et Homo floresiensis (Zeitoun et al. 2010).

L'Indonésie et ses myriades d'îles et îlots (environ 14000) s'inscrivent dans un rectangle de $5000 \mathrm{~km}$ sur $2000 \mathrm{~km}$ de part et d'autre de l'équateur entre $5^{\circ} 00 \mathrm{~N}$ et $120^{\circ} 00 \mathrm{E}$ ce qui en fait le plus grand archipel du monde (Figure 1). Cette aire géographique immense s’étirant sur un espace maritime d'environ 6 millions de $\mathrm{km}^{2}$, ne nous permet pas aujourd'hui de traiter exhaustivement l'ensemble des groupes industriels, des faciès ou des cultures préhistoriques, c'est pour cela que nous aborderons les principaux.

Rares sont les technocomplexes bien définis avant l'Holocène, période où l'insularité de cette aire géographique s'est fixée avec la remontée marine marquant progressivement le début de l'histoire des archipels insulindiens. Parmi ceux-ci nous citerons le «Toalien » à Sulawesi (faciès à pointes), le "Sampungien » (faciès à pointes) et les industries sur éclats de Song Keplek (débitage orthogonal) dans l'Est de l'île de Java. Hormis ces trois traditions techniques qui restent individualisables sur un plan typo-technologique, il n’y a pas dans l'ensemble du matériel rencontré, de faciès différentiables associés à une dénomination d’outils spécifiques. En règle générale, les industries lithiques du Pléistocène supérieur et de l'Holocène indonésien répondent à une production basique d'éclats et d'outils sur éclats avec de multiples variantes régionales. Le mode de débitage est très largement à la pierre dure, non Levallois, rarement Discoïde, non lamino-lamellaire tel qu'on peut le rencontrer en Europe de l'Ouest ou au Proche et Moyen-Orient.

Conformément à la devise du pays « Bhinneka tunggal ikat » (l’unité dans la diversité), les industries préhistoriques indonésiennes s'enracinent dans un fonds tourné majoritairement vers une production d'éclats très épais, malgré quelques exceptions (Toalien, Sampungien) venant rompre une certaine somnolence de formes de supports-outils (Forestier et al. 2010). Il 
en découle une difficulté à décerner des variantes sur un plan synchronique comme diachronique. Ce problème de classification, d'individualisation et de détermination chronoculturelle est souvent évoqué et récurrent dans les études lithiques en Asie du Sud-Est renvoyant à un dialogue entre le déterminisme géographique et les choix culturels : "In Europe as well as in the Americas, typology of at least some key forms was and still is the basis of relative chronology. (...) Is the typological approach not appropriate to the special characteristics and circumstances of lithic industries in Southeast Asia? Why do we know of only a few formal «Asian » types and how did the availability and acquisition of raw material influence lithic technology? Are morphological features of lithics artefacts significant enough at all to establish an acceptable chronology system of lithic periods? How can morphological features of lithic artefacts be linked to geostratigraphy in Southeast Asia? Can non-stratified surface finds contribute to Palaeolithic and Neolithic chronologies in that region? And if we dismiss, the typological approach, what are the alternatives? " (Haidle \& Pawlik 2009: 2). Une des solutions pour sortir de cette ornière épistémologique est selon nous l'approche technologique par le diagnostic systématique des méthodes et des techniques de taille doublée si possible, d'une approche morpho-fonctionnelle des supports-outils (Borel 2012; Borel et al. 2013).

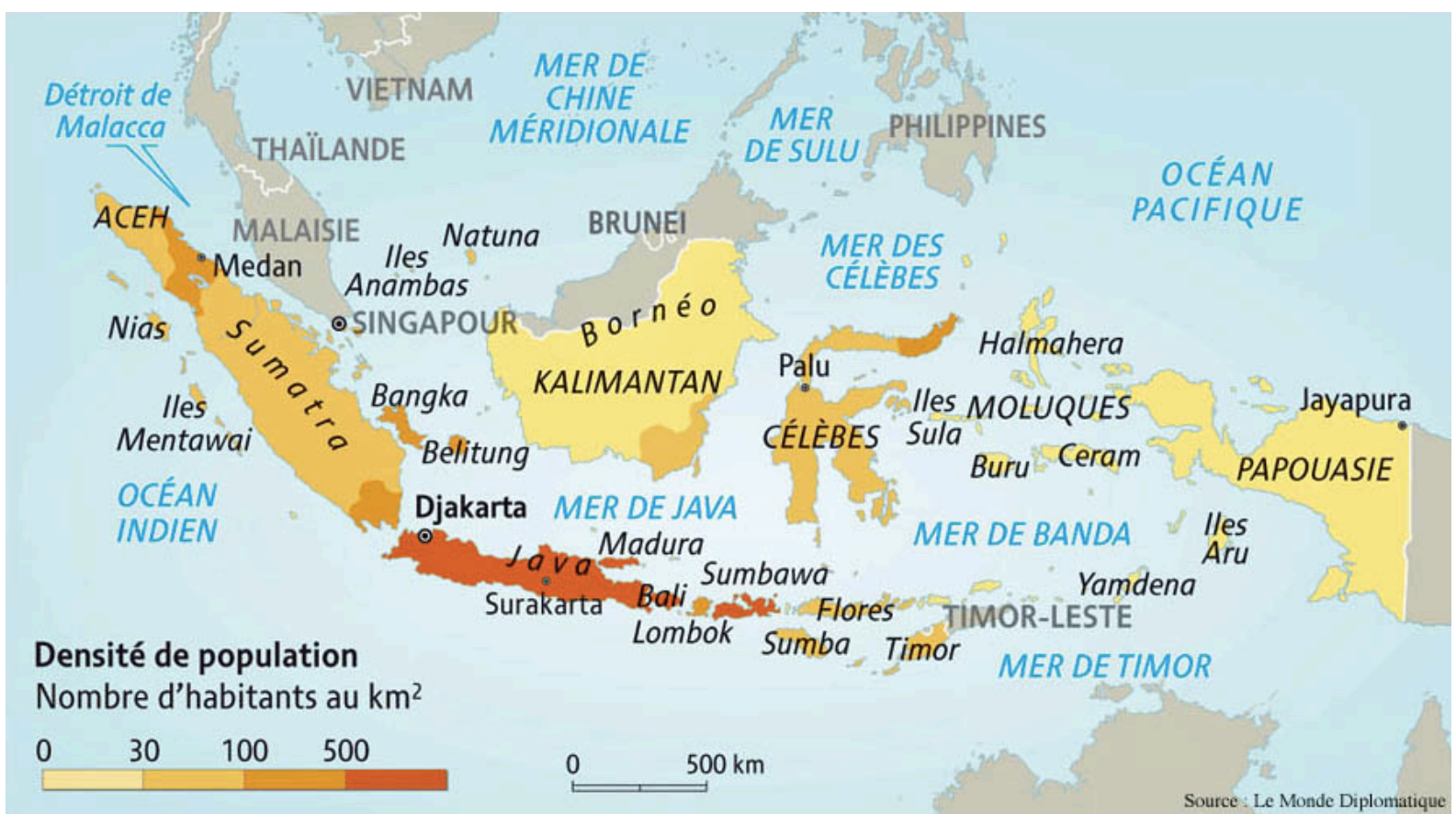

Figure 1. Situation géographique de l’Indonésie (adapté d’après Kristianasen 2010).

Figure 1. Geographical location of Indonesia (adapted after Kristianasen 2010).

Le but de cet article est donc de présenter de façon synthétique les principaux ensembles lithiques de l'Archipel indonésien à partir d'une sélection d'îles sur lesquelles des assemblages lithiques ont pu être correctement documentés contextuellement, taphonomiquement, techniquement et chronologiquement. Nous nous intéresserons ainsi aux îles de Sumatra, de Java, de Kalimantan (Bornéo), de Sulawesi, et à quelques autres plus orientales qui, comme Timor et Flores, ont, ces dernières années, livré des découvertes de premier plan. Par commodité, nous avons été obligés de procéder à des coupures régionales et chronologiques (Pléistocène ancien-moyen et Pléistocène supérieur final-Holocène ancien) qui permettent d'exposer au mieux cette synthèse sur des assemblages lithiques qui n'ont pas tous fait l'objet d'études technologiques approfondies au sens où nous pouvons l'entendre en Europe avec l’utilisation du concept de chaîne opératoire. 
D’un point de vue chronologique, la majorité des assemblages lithiques que nous mentionnerons dans ce texte remonte au Pléistocène supérieur final et au début de l'Holocène mais nous indiquerons quelques éléments propres à la culture matérielle des premiers peuplements qui sont beaucoup plus anciens (culture matérielle des Homo erectus).

Les premiers assemblages cités correspondent au «Paléolithique supérieur et au Mésolithique ». Si cette attribution peut trouver un écho chronologique, elle n’a aucune valeur chrono-typologique ou technologique rappelant, bien souvent davantage des types d'outils paléolithiques d'aspect très « moustéroïde ».

A l'instar de l'Asie du Sud-Est continentale (voir article Forestier et al. 2017, ce numéro), le modèle européen tourné vers un allégement généralisée des supports d'outils, des changements de méthodes et de techniques de taille bien marqués suivant le fil du temps et une idée graduelle du progrès technique, est loin d'être applicable pour l'Indonésie. Comme nous allons le voir, la particularité et l'originalité de la préhistoire indonésienne reposent d'une part sur la difficulté de distinguer un Paléolithique inférieur, moyen et supérieur et d'autre part, sur la question de l'homogénéité et de l'hétérogénéité des assemblages à comparer.

Compte tenu du fait que la très grande majorité des analyses des séries lithiques menées en Indonésie n’a pas été guidée par le concept de chaîne opératoire, il nous a paru plus prudent de présenter la variabilité du matériel connu et accessible au cas par cas, et d'en tirer une expertise qualitative portant sur les méthodes et techniques de taille.

\section{Paléogéographie de l’Indonésie quaternaire : de péninsules en archipels.}

La géographie de l'Indonésie résulte d'une paléogéographie complexe, influencée par l'eustatisme et le volcanisme d'une zone à forte activité tectonique et sismique. Les variations du niveau marin durant le Quaternaire ont permis l'accès intermittent aux sous-continents Sunda et Sahul, ce qui en a conditionné et favorisé le peuplement (Figure 2). Cinquante mètres de baisse du niveau marin suffisent pour que les îles de Sumatra, Java et Kalimantan forment une entité terrestre (le Sunda) rattachée à l'Asie péninsulaire (Van den Bergh et al. 1996). Cette baisse de $50 \mathrm{~m}$ a été effective durant presque la moitié des derniers 250000 ans BP (Voris 2000). Pendant le dernier maximum glaciaire (LGM) et jusqu'à environ 11000 ans BP, des populations d'hommes modernes ont pu se déplacer à pied sec entre l'Asie du SudEst continentale et l'Indonésie à l'époque où la mer de Java était une plaine.

Durant la transition Pléistocène-Holocène vers 11000 BP et le début de la remontée de eaux, la surface émergée a diminué progressivement d'un tiers (Voris 2000). Sumatra est devenue réellement une île isolée de la péninsule malaise et de Kalimantan aux alentours de

6000 ans BP (Sathiamurthy \& Voris 2006; Hanebuth et al. 2011). Le milieu de l'Holocène marque pour ainsi dire l'aspect de l'Archipel indonésien actuel.

À toutes les époques, l'histoire des hommes et de leurs techniques est influencée par des événements paléo-climatiques et paléogéographiques majeurs impliquant l'apparition et la disparation de niches et de barrières écologiques. Des phénomènes internes de radiation, d'isolat, d'endémisme ou encore, par exemple, de résilience vont autant intervenir sur le vivant que sur la technique ou les modes de vie de groupes insulaires. Cela implique un fort déterminisme sur les ressources donc sur les modes de subsistance et les comportements des groupes qui se sont succédés depuis le peuplement initial de l'Archipel par Homo erectus il y a un peu plus de 1 million d'années si l'on s'accorde sur la datation obtenue par trace de fission de $\leq 1,4$ million d'années du crane de Modjokertol découvert sans matériel lithique associé (Morwood et al. 2003; O’Connell \& De Silva 2013).

Concernant l'arrivée ancienne des premiers Homo sapiens en Indonésie, elle reste encore incertaine et hypothétique (Polanski et al. 2016) même si certaines études suggèrent une 
première arrivée probable vers 80000-100000 BP (Storm et al. 2005). Pour l'heure, les fossiles attestent une présence seulement à partir d'environ 37000-28000 ans BP (cf. la récente datation de l’homme de Wadjak par U-series; Storm et al. 2013).

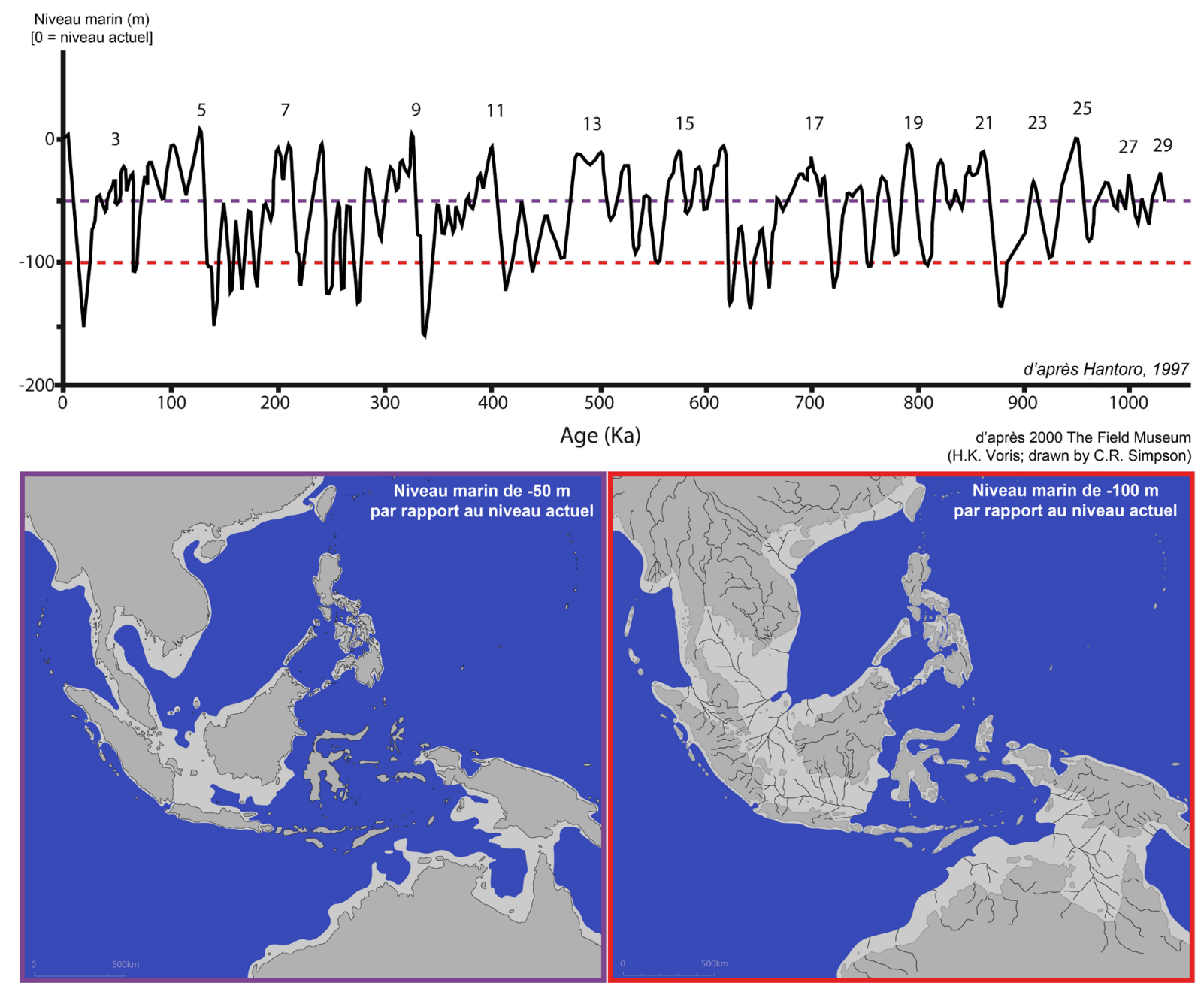

Figure 2. Courbe de variation du niveau marin au cours du dernier million d'années et cartographie des terres émergées lors de la régression marine de - $50 \mathrm{~m}$ (à gauche) et de - $100 \mathrm{~m}$ (à droite). (d'après Voris 2000).

Figure 2. Sea level variation during the last 1 million years and map of the emerged land during sea level drop of $50 \mathrm{~m}$ (on the left) and of $100 \mathrm{~m}$ (on the right). (after Voris 2000).

Les conditions les plus favorables pour que des hommes modernes atteignent l'Australie correspondent à la phase où le sous-continent Sunda était relié à l'Asie du Sud-Est continentale c'est-à-dire à une baisse importante du niveau marin telle que celle enregistrée entre 70000 et 60000 BP d'après les isotopes de l'oxygène de la péninsule Huon en Papouasie Nouvelle-Guinée mais les preuves d'activités humaines ne sont attestées qu'à partir de 49000 ans BP (Summerhayes et al. 2010).

Cet événement corrobore les dates obtenues pour le peuplement initial de l'Australie daté directement sur les sites soit autour de 60000 ans BP (Bowler et al. 2003). Cette datation a été obtenue par TL à Malakunanja II (Roberts et al. 1990; Clarkson et al. 2015) et Nauwalabila I (Roberts et al. 1994). Cette limite chronologique du peuplement de l'Australie et le franchissement du Sahul restent très éloignés de la date de 120000 BP annoncée pour le site de Jinmium (Fullagar et al. 1996). Cette datation a d'ailleurs été ensuite abaissée à 22000 BP par Roberts et al. (1998). 
Plusieurs sites chinois datés entre 150000 et 80000 ans sont marqués par la présence de Homo sapiens comme à Jinniushan (Braüer \& Mbua 1992), Chaoxian (Xu et al. 1984) et Maba (Zhou et al. 1982; Wu 1983). La mandibule de Zhirendong (Liu et al. 2010) ainsi que les restes de Liujiang à Tongtianyan (Woo 1959) sont considérés comme les plus anciens hommes modernes (Shen et al. 2002) avec des dates respectivement de 100000 ans (Liu et al. 2010) et 153000 ans (Shen \& Michel 2007). Aux Philippines, à Calao Cave l'homme moderne serait présent dès 67000 ans BP ce qui en fait à l'heure actuelle le fossile le plus ancien d'Asie du Sud-est insulaire (Mijares et al. 2010).

\section{Les productions très anciennes en Indonésie centrale il y a environ 1 million d'années}

Le Pléistocène ancien est une période où les outils découverts sont très importants car ils sont rares et sont concomitants de la présence des Homo erectus en Indonésie il y a au moins 1 million d'années (Widianto et al. 2001; Stone 2006; Van Heteren \& de Vos 2012; Sémah et al. 2014b). Toutefois, pour cette période très reculée les sites bien datés et bien stratifiés sont peu nombreux comme par exemple celui de Ngebung 2 fouillé par la Mission «Quaternaire et Préhistoire en Indonésie » (Sémah 2001; Sémah et al. 2002). Si l'on ne s’autorise pas encore à parler stricto sensu de site acheuléen pour cette période comme on pourrait le faire plus facilement en Europe de l'Ouest, au Proche-Orient voire même en Asie du Sud (Dennell 2009), toute une gamme d'outils très divers sur quartz ou roche métamorphique fait état d'une industrie archaïque et originale à la fois (Soejono 1979).

A Ngebung 2, bola, hachereau, large éclat (LCT = Large Cutting Tool) et chopper ont été enregistrés sur un sol dit de la formation de Kabuh daté de 800000 ans BP par les méthodes ESR, U-Th et Ar/Ar (Sémah 2001; Brasseur et al. 2011). Cette industrie est à ce jour la plus ancienne découverte en Indonésie en contexte archéo-stratigraphique clair et bien daté avec celle mise au jour à Flores (Mata Menge). Elle est associée à une faune ancienne dans laquelle on retrouve, entre autre, un Axis lydekkeri ngebungensis (Cervidae) et un Stegodon (Sémah et al. 1992; Moigne et al. 2004).

D’autres sites ont livré des pièces anciennes parfois spectaculaires comme des bifaces et des hachereaux, mais pour beaucoup d'entre eux, il s'agit de sites de plein air, mal ou pas datés en contexte pédo-stratigraphique peu fiable. On les trouve le long des berges de rivières, sur des terrasses alluviales ou dans le lit de cours d'eau en situation de mélange alluvionnaire (Figure 3). Ces derniers sont parfois devenus des sites éponymes de renom comme le «Padjitanien » découvert dans la rivière Baksoko non loin de la ville de Pacitan à Java Est (Bordes 1968; Bartstra 1976; 1982). Des industries à biface ont aussi été identifiées dans la rivière Ogan à Sumatra Sud (Forestier 2007a) (Figures 4 et 5) ou dans la rivière Wallanea (Cabenge) au Sud-Ouest de Sulawesi qui a été récemment sondée et datée entre 200000 et 100000 ans (Keates \& Bartstra 2001; Keates 2004; Van den Bergh et al. 2016a).

Grand nombre de pionniers de la préhistoire paléolithique en Indonésie comme G. H. R. Koenigswald, H. R. Van Heekeren, R. P. Soejono, G. J. Bartstra se sont penchés sur la question des outils de Homo erectus et sur l'existence ou non d'un faciès Acheuléen sur la base de la présence-absence de bifaces et de hachereaux. Un exercice qui a été appliqué sur des pièces identiques à celles découvertes sur les îles de Sumatra (Forestier 2007a), de l'Est de Java (Sémah et al. 2014b) et de Sulawesi (Keates \& Bartstra 2001; Simanjuntak et al. 2010). Or, si ce matériel apparaît sur Java vers 800000 ans avec d'évidentes normes acheuléennes, il n'en demeure pas moins singulièrement « indonésien » (ou asiatique) avec des morphologies assez archaïques par rapport aux pièces africaines ou indiennes qui déploient des formes variées et évolutives sur le temps long (Simanjuntak \& Forestier 2008; 2009; Brumm \& Moore 2012). 


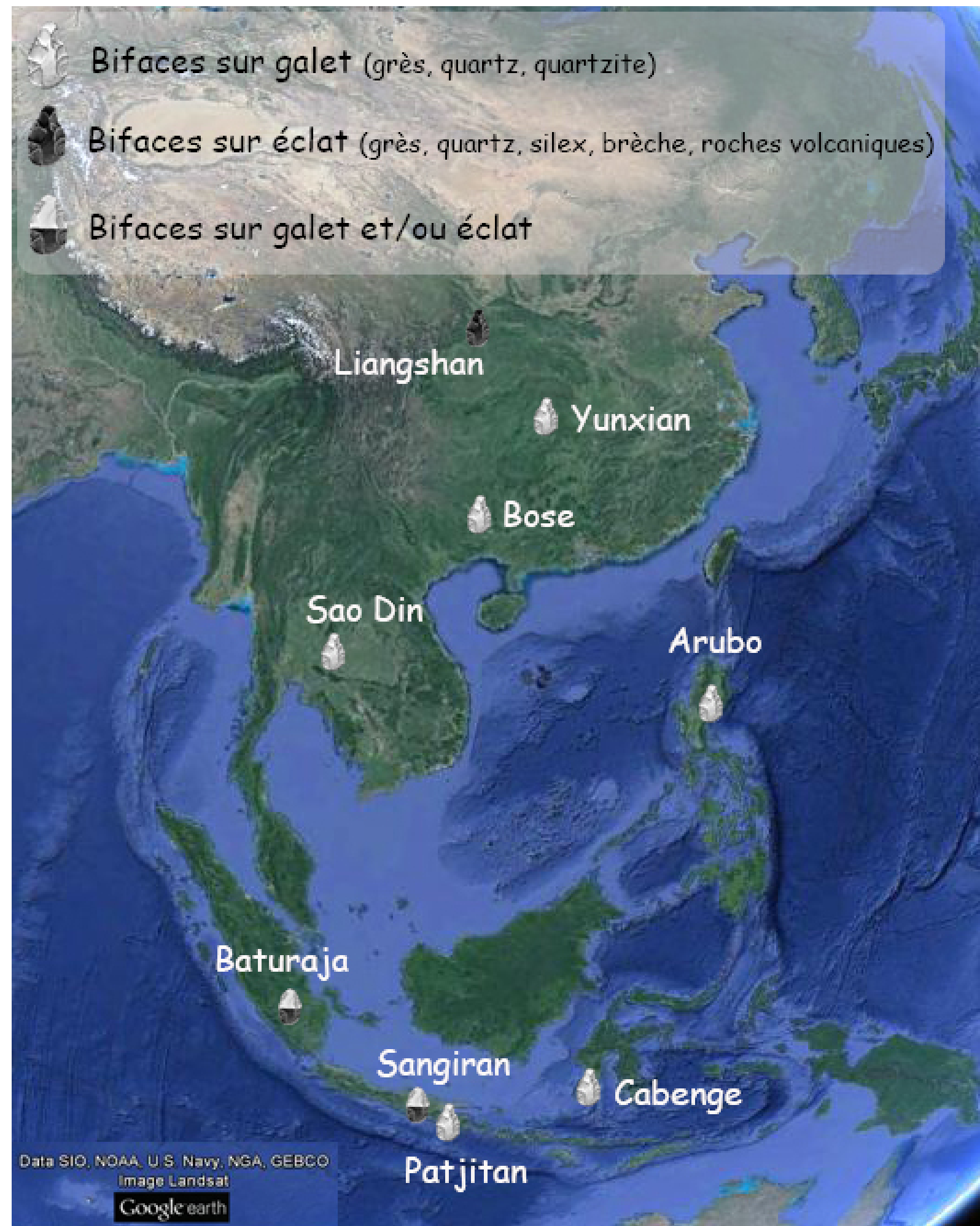

Figure 3. Répartition géographique des bifaces en Asie du Sud-Est et de l'Est. (Forestier) (Image: Data SIO, NOAA, U.S. Navy, NGA, GEBCO, Image Landsat, Goofle earth).

Figure 3. Geographical distribution of hand-axes in Southeast Asia and East Asia. (Forestier) (Image: Data SIO, NOAA, U.S. Navy, NGA, GEBCO, Image Landsat, Goofle earth). 

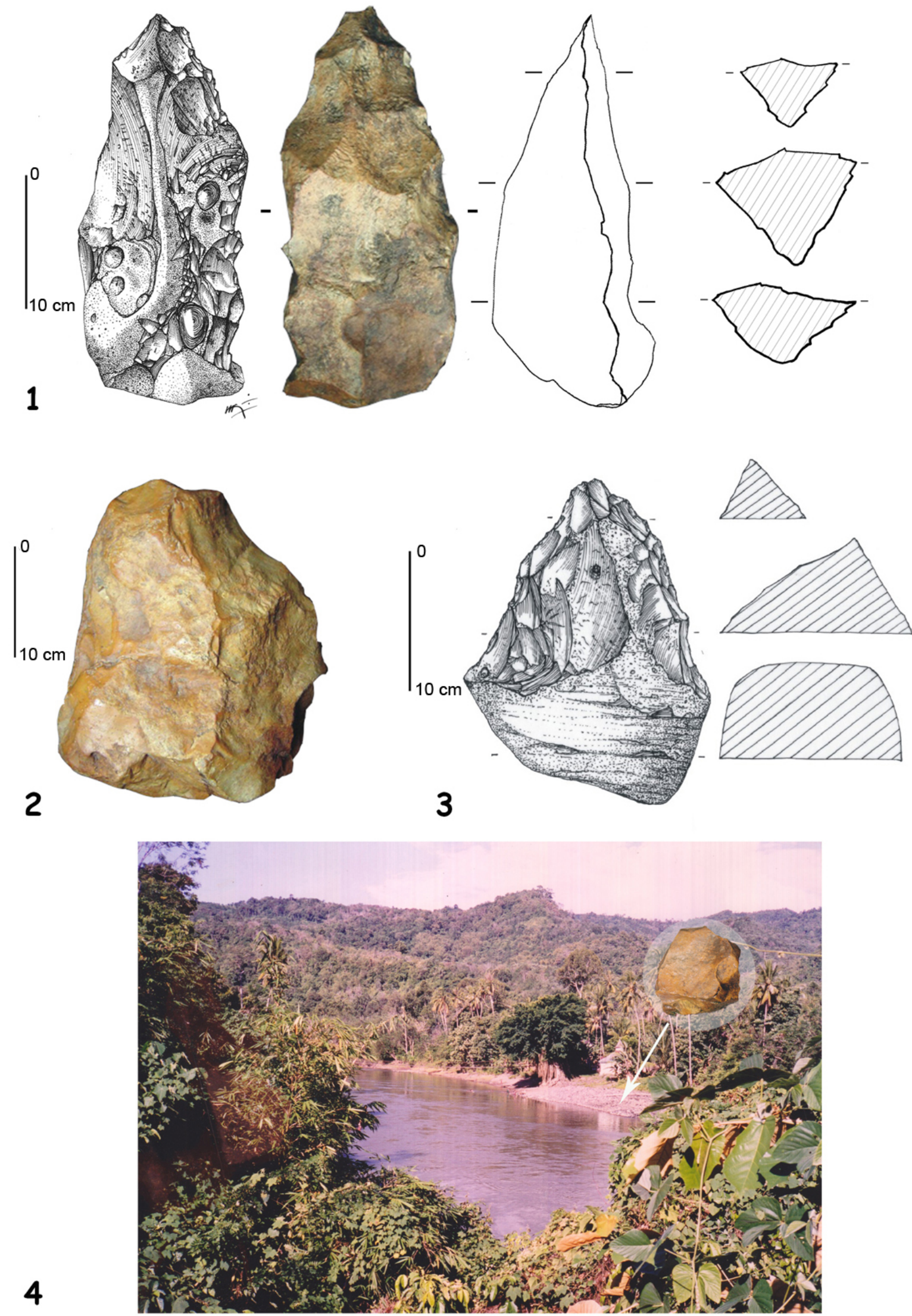

Figure 4. Biface de la rivière Ogan, Sumatra Sud. 1, 2 : biface ; 3 : chopper convergent (pointu) ; 4 : vue de la rivière Ogan et bloc de chert. (Forestier).

Figure 4. Hand-axe of Ogan River, South Sumatra. 1, 2: hand-axe ; 3: pointed chopper ; 4: view of the Ogan river and chert raw material block. (Forestier). 

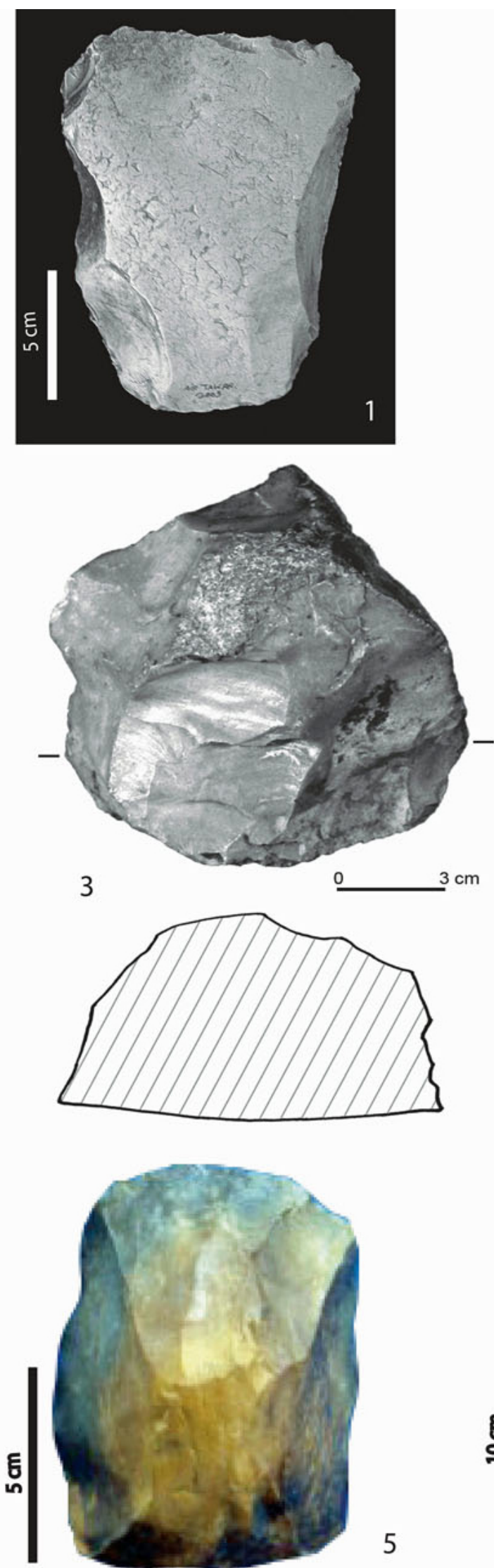

5
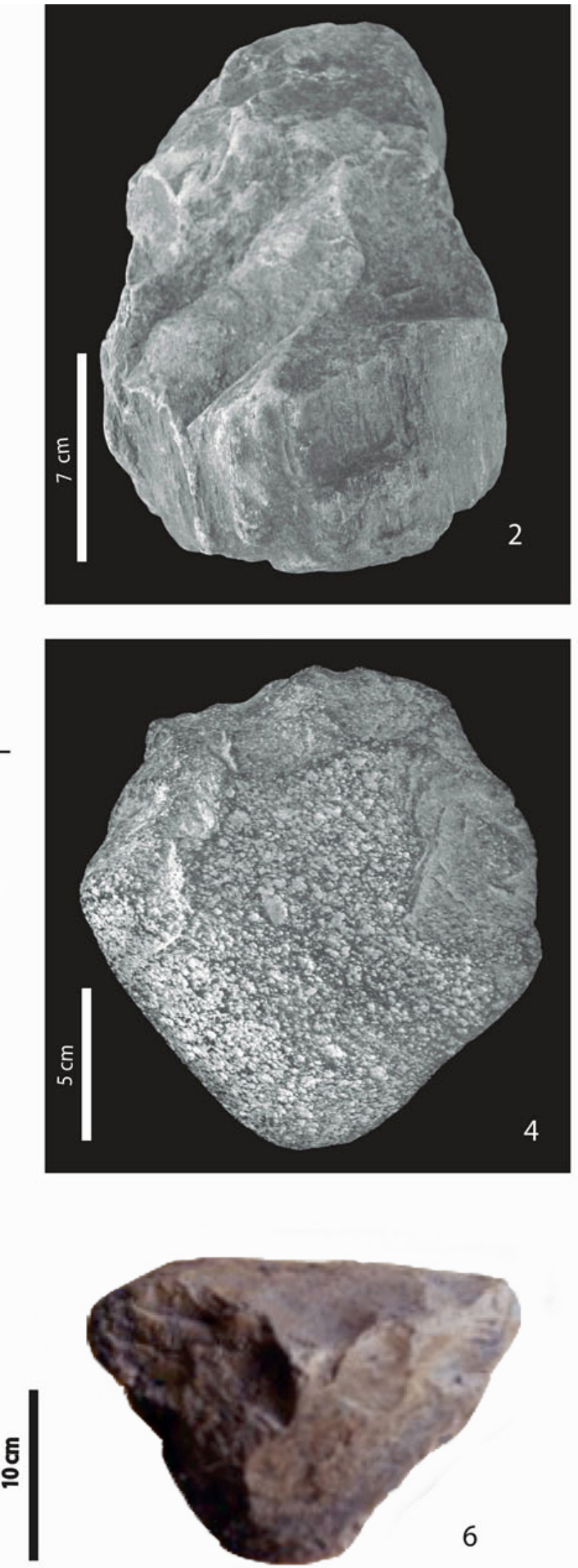

6

Figure 5. Outils paléolithiques de la rivière Ogan, Sumatra Sud. 1 : hachereau ; 2 : pic triédrique (bois silicifié) ; 3, 5 : rabot à front épais ; 4 : chopper ; 6 : nucléus. (Forestier).

Figure 5. Palaeolithic tools of Ogan River, South Sumatra. 1: cleaver ; 2: triedrical pick (silicified wood) ; 3, 5: rabot with thick front edge ; 4: chopper; 6: core. (Forestier). 
Ce décalage de près de 1 million d'années ne signifie en rien qu'il y a eu une vague migratoire de l'Afrique vers l'Indonésie mais pose la question de la phylogénie de ces pièces asiatiques. Quelque soit la route prise par les hominines (e.g., Reyes-Centeno 2016) soit par le Nord via la Chine et l'Asie du Sud-Est péninsulaire ou bien, par le Sud via l'Inde-Penjab, le Bengladesh, la côte birmane en direction de l'île de Sumatra puis celle de Java, on constate que des mouvements de populations sont possibles dans divers sens sans exclure la possibilité de réinventions locales.

Deux raisons président à ces observations. La première d'ordre techno-logique est avancée par l'Indonésie où tous les stades évolutifs des bifaces n’ont, à ce jour, pas été retrouvés. Bifaces et hachereaux apparaissent vers 800000 ans et restent figés à un niveau technique sur plusieurs millénaires (mode clos, hypertélique) comme une réponse adéquate et définitive au milieu dans lequel l'objet entame un processus d'adaptation. Cette stabilité morpho-fonctionnelle pourrait être vue dans ce cas, comme une preuve d'adaptation réussie au milieu.

La seconde interroge la non-synchronie de ce matériel intra-inter-îles comme par exemple la variation des types d'outils rencontrés d'une île à l'autre ; ou lors des phases de régressions marines, la différence qui peut exister entre les sites de l'extrémité d'un super continent exondé (cul-de-sac javanais - bordure orientale du Sunda) et ceux des îles isolées de la Wallacea (Sathiamunthy \& Voris 2006; Sémah et al. 2014a).

Au Pléistocène ancien sur Java se retrouvent associés hachereaux, éclats et bolas (Sémah et al. 1992; Simanjuntak et al. 2010; Sémah et al. 2014b ) ; alors qu’à Sumatra des bifaces très massifs façonnés sur bloc côtoient des chopper, des LCT et des hachereaux sur éclats (Forestier et al. 2005a). Quant à l'île de Sulawesi elle se distingue des deux précédentes avec des bifaces façonnés sur de gros galets ovoïdes et des choppers-chopping-tools (Keates \& Bartstra 2001).

À ces deux raisons s'ajoutent des nuances propres aux diversités techniques, morphologiques de chaque région mais aussi aux matières premières rencontrées et aux objectifs des tailleurs. Autant de facteurs paléogéographiques qui expliqueraient des évolutions, de l'isolation traduisant un phénomène de redécouverte ou de convergence, comme le faisait déjà remarquer à son époque F. Bordes à propos des assemblages à biface de Java : "Un cas intéressant est celui du Padjitanien, industrie comportant des bifaces typiques mais isolée en cul-de-sac à Java, sans lien actuellement connus avec la zone à biface la plus proche, l'Inde » (1968: 136).

L'idée bifaciale se présente comme un possible parmi d'autres : l'option technique d'un lieu, d'un territoire, d'une île en accord avec un environnement et les besoins qu'il suscite. Elle inclut dans sa conception une richesse d'expression de forme, de volume, d'utilisation préférentielle des bords (tranchants), qui permet à chaque artisan de s'exprimer de façon différente ouvrant ainsi à une variabilité dans une recherche du convergent, de l'apical et du pointu.

Cependant, sur un plan diachronique, cette idée technique n’est pas toujours attachée aux périodes très anciennes de l'archipel, sans vraiment de limite de temps et d'espace puisqu'on la perd grosso modo aux confins du Pléistocène moyen pour la retrouver ensuite au Pléistocène supérieur final. C’est le cas des bifaces retrouvés au Nord de Kalimantan dans le territoire du Sabbah (Malaisie) dans le site de plein air Tingkayu daté entre 28000 et 18000 ans BP (Bellwood 1988; 2007; Saidin \& Jeffrey 2014). Mais également dans d'autres îles dans d'autres archipels tels que les sites de plein air philippins comme Cagayan Valley et Arubo sur l'île de Luzon (Pawlik 2001; 2004; Pawlik \& Ronquillo 2003; Dizon \& Pawlik 2010).

Hormis quelques pièces exceptionnelles de très grande dimension $(\geq 20 \mathrm{~cm})$ comme celles de Sumatra Sud réalisées à partir de méga-blocs de silex (chert) qui témoignent d'un 
façonnage parfois bien maîtrisé, les bifaces d’Asie du Sud-est sont généralement peu soignés, de modeste facture comme le montre la Figure 4. Ils se trouvent associés à des nucléus prismatiques massifs de plusieurs dizaines de kilos, de larges éclats, des choppers, des hachereaux et autres rabots à front épais (Figure 5).

De manière générale et d'après les pièces que nous avons pu observer, les bifaces d'Asie du Sud-Est sont partiellement façonnés, corticaux, très épais, peu standardisés et très souvent difficilement classables sur un plan morpho-typologique. L'arête du tranchant de ces pièces épaisses est souvent sinueuse et marquée par des segments non aménagés laissés corticaux à nombreuses cassures traduisant des accidents de taille périphériques.

Dans ces régions tropicales, le façonnage partiel à la percussion directe à la pierre dure apparaît comme une des caractéristiques technologiques. La morphologie du support d’origine (grand éclat, bloc ou galet) y est peu modifiée au cours de la taille. Cela indique une chaine opératoire de façonnage assez courte, sans étape de préforme ou d'ébauchage, réalisée par percussion directe (rentrante) avec seulement trois à quatre générations d'enlèvements. Les éclats de façonnage ont les caractéristiques suivantes : épais, plus large que long, talon non préparé souvent cortical ou semi-cortical, profil très arqué, face supérieure corticale avec des enlèvements antérieurs préférentiellement unidirectionnels.

Dans ce cas, l'absence d'étape d'ébauchage raccourcit la chaîne opératoire et empêche la structuration stricto sensu de deux surfaces répondant d'un plan d'équilibre bifacial qui est que très rarement atteint. D'ailleurs, les sections des bifaces sont très rarement biconvexes, biplan convexes comme c'est le cas pour les productions bifaciales plus classiques où la taille est mieux maîtrisée, davantage hiérarchisée selon des règles techniques de changement entre ébauchage et finition (changement de technique de percussion possible : le passage percuteur dur au percuteur dur-tendre ou hard-soft hammer). Les courtes séquences de façonnage à enlèvements peu envahissants expliquent les larges plages corticales résiduelles très présentes en partie mésiale et proximale mais également, les sections qui sont généralement triangulaires ou trapézoïdales. Les bifaces sud-est asiatiques peuvent passer pour n'être pas très orthodoxes si on les compare aux pièces européennes, africaines ou indiennes car ils sont peu «élaborés » ou "élémentaires » avec un faible investissement technique (absence de retouche totale envahissante et de plan d'équilibre bifacial et bilatéral).

Façonnés souvent sur des matières premières non siliceuses et sur des galets de forme oblongue, les bifaces du Sud-Est asiatique n’ont pas nécessité le même investissement technique qu'en Eurasie et en Afrique (le même «soin à la taille »), mais respectent néanmoins trois critères techniques qui en font des bifaces : l'allongement, l'extension du tranchant et la symétrie même si elle est imparfaite selon les canons européens.

\section{Plus à l'Est vers les petites îles de la Sonde : d'autres traces très anciennes d'activités}

\subsection{Les industries lithiques du centre de Flores (bassin de So'a, Mata Menge)}

C’est en 1957 que Th. Verhoeven découvre sur l'île de Flores, dans la région de Ola Bula (bassin de So'a, bassin versant de la rivière Ae Sissa, Centre Flores) des os fossiles attribuables à une espèce fossile de proboscidien estimée au Pléistocène. Comparable à celui mis au jour à Java, ce Stegodon a été dénommé Stegodon trigonocephalus (Hooijer 1957; Verhoeven 1958) puis Stegodon trigonocephalus florensis (Hooijer 1964; 1972) et finalement requalifié de Stegodon florensis par Van den Bergh en 1999. Qualifiées au départ de «blades and flakes» (Verhoeven 1968), ces industries lithiques avaient été systématiquement collectées et associées aux vestiges fauniques en surface comme en fouilles (Hartono 1961; Maringer \& Verhoeven 1970c; Van Heteren \& De Vos 2012). Dès 1965, toujours dans la zone de Mengeruda, à Mata Menge puis à Lembahmenge, des fouilles ont permis de récolter une série d'artefacts en grande majorité en andésite dans un niveau fossilifère à Stegodon 
datés par tektite de 500000 à 830000 ans (Maringer \& Verhoeven 1970a; 1970b; 1970c). Les artefacts collectés ont été dits similaires à ceux découverts à Sangiran sur l'île de Java par von Koenigswald et Ghosh (Von Koenigswald \& Ghosh 1973) et confortent l'hypothèse de l'existence d'un homme fossile (Homo erectus) dès le Pléistocène moyen sur l'île de Flores (Maringer \& Verhoeven 1970c; 1975).

D'un point de vue typologique les industries de Mengeruda et d'Ola Bula ont été qualifiées d'industries à éclats sans biface (Van Heteren \& De Vos 2012). D’un point de vue technologique, la description effectuée par Maringer \& Verhoeven est déjà suffisamment explicite et précise pour déduire au moins des modalités de débitage de type orthogonal (ou SSDA - Système par Surface de Débitage Alternée) c'est-à-dire reposant sur une organisation à plans de frappe multiples orthogonaux inscrits dans un système de surfaces de débitage alternées entretenu par un "algorithme dit de base " (Forestier 1993). L'utilisation d'une modalité discoïde du débitage telle que définie par Boëda (1993) est plus rare alors que l'emploi d'une méthode Levallois est absent.

Depuis ces travaux pionniers, d'autres collectes d'artefacts ont eu lieu in situ à Mata Menge ainsi que des prélèvements pour datation (Sondaar et al. 1994; Morwood et al. 1997; 1999; Van den Bergh 1997; Van den Bergh et al. 1996; O’Sullivan et al. 2001). Les dates obtenues par la méthode des traces de fission de l'Uranium donnent une estimation optimale de l’âge des dépôts fossiles à Mata Menge entre 880000 et 800000 ans (Morwood et al. 1998; O'Sullivan et al. 2001; Brumm et al. 2010; Van den Bergh et al. 2014).

L'analyse techno-typologique proposée ces dernières années (Brumm et al. 2010) sur le niveau principal à fossiles et artefacts de Mata Menge (Unité B) comporte assez peu de pièces ( $n=556)$ et confirme les deux méthodes de taille énoncées plus haut. Le débitage est un débitage d'éclats réalisé à partir de blocs arrondis en roche volcanique ou de galets. Les nucléus sont majoritairement de direction centripète discoïde ( «radial » cores, 9\%) et dans une moindre proportion à plan de frappe multiples (multiplatform cores, 2,2\%) (Brumm et al. 2010: 456 et 459) (Figure 6). Certains d'entre eux totalement épuisés ont pu être repris en outils ou core tools (Moore \& Brumm 2007).

Ainsi, plusieurs modalités de débitage ont été mises en œuvre à Mata Menge avec une préférence pour les débitages d'orientation centripète proches d'un schéma discoïde associés à d'autres moins contrôlés, davantage orientés vers un débitage orthogonal (multiplatform cores).

La récente découverte dans le Bassin de So’a dans le centre de l'île de Florès de restes attribuée à Homo floresiensis (morceau de mandibule ; Van den Bergh et al. 2016b) pourrait indiquer qu'il s'agit bien de l'auteur de cette industrie dès 800000-700000 ans BP (Brumm et al. 2006). Les datations utilisées sont des datations Argon et par traces de fission pour le niveau cendreux d'origine volcanique contextualisant les fossiles retrouvés et des datations Useries et ESR sur les dents fossilisées.

\subsection{Artefacts et petits hommes à Liang Bua}

La découverte de Homo floresiensis à Liang Bua (Brown et al. 2004; Morwood et al. 2004) offre la possibilité d'étudier les productions lithiques de cette espèce humaine endémique de Flores, considérée comme une descendance évolutive naine des Homo erectus régionaux (Lyras et al. 2009; Zeitoun et al. 2016). Les artefacts associés à Homo floresiensis (Figure 7) et à une faune à Stegodon florensis (secteurs IV et VII) montrent un débitage d'éclats simples, d'aspect centripète ce qui donne des supports hétérogènes parfois allongés d'aspect laminaire (Morwood et al. 2005). 

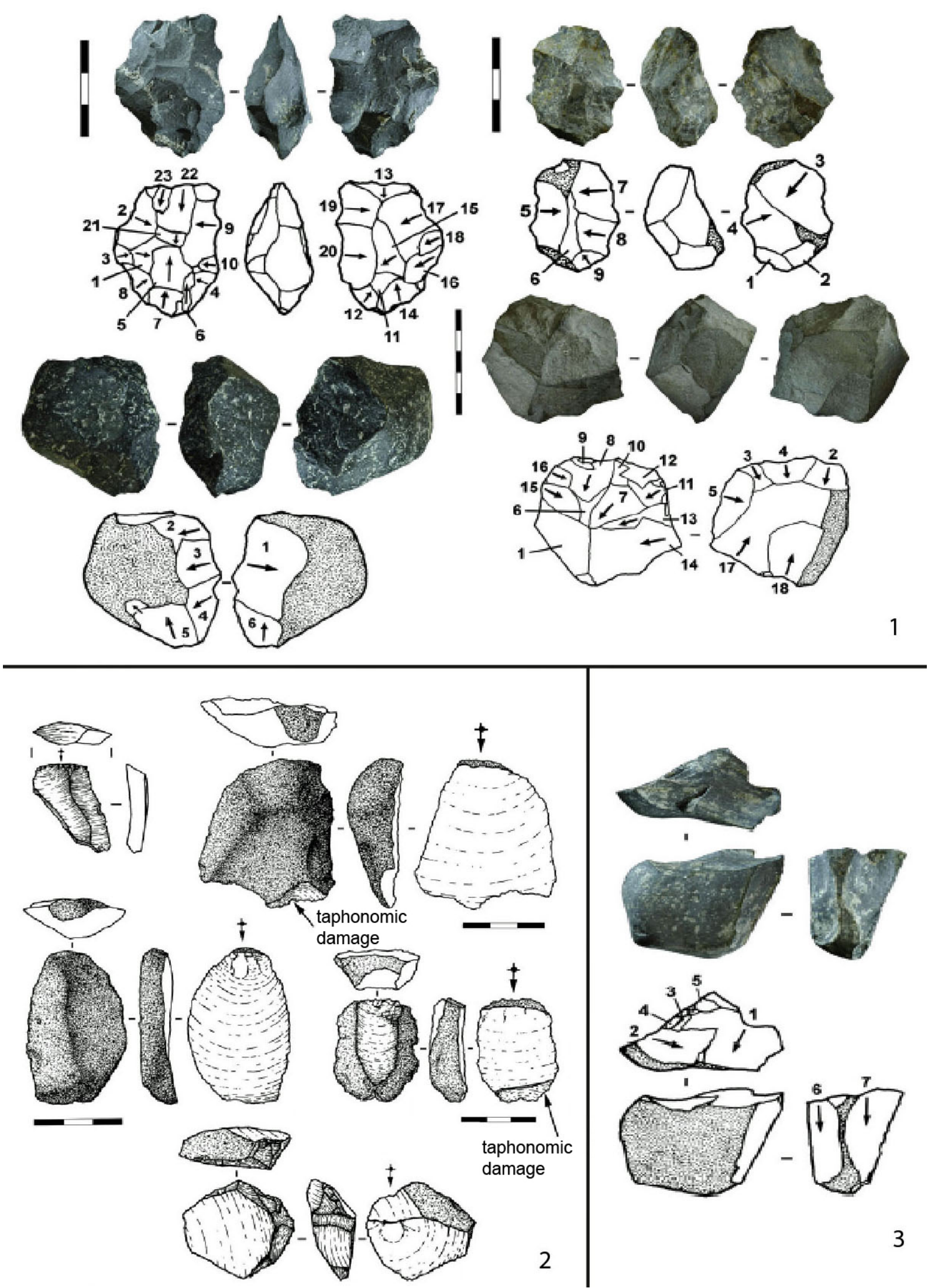

Figure 6. Industrie lithique de Mata Menge, Bassin de So’a, Florès. 1: nucléus discoïdes; 2: éclats; 3: nucléus orthogonal. (d'après Brumm et al. 2010).

Figure 6. Lithic industry of Mata Menge, So’a Basin, Flores. 1: discoidal cores; 2: flakes ; 3: orthogonal core. (after Brumm et al. 2010). 

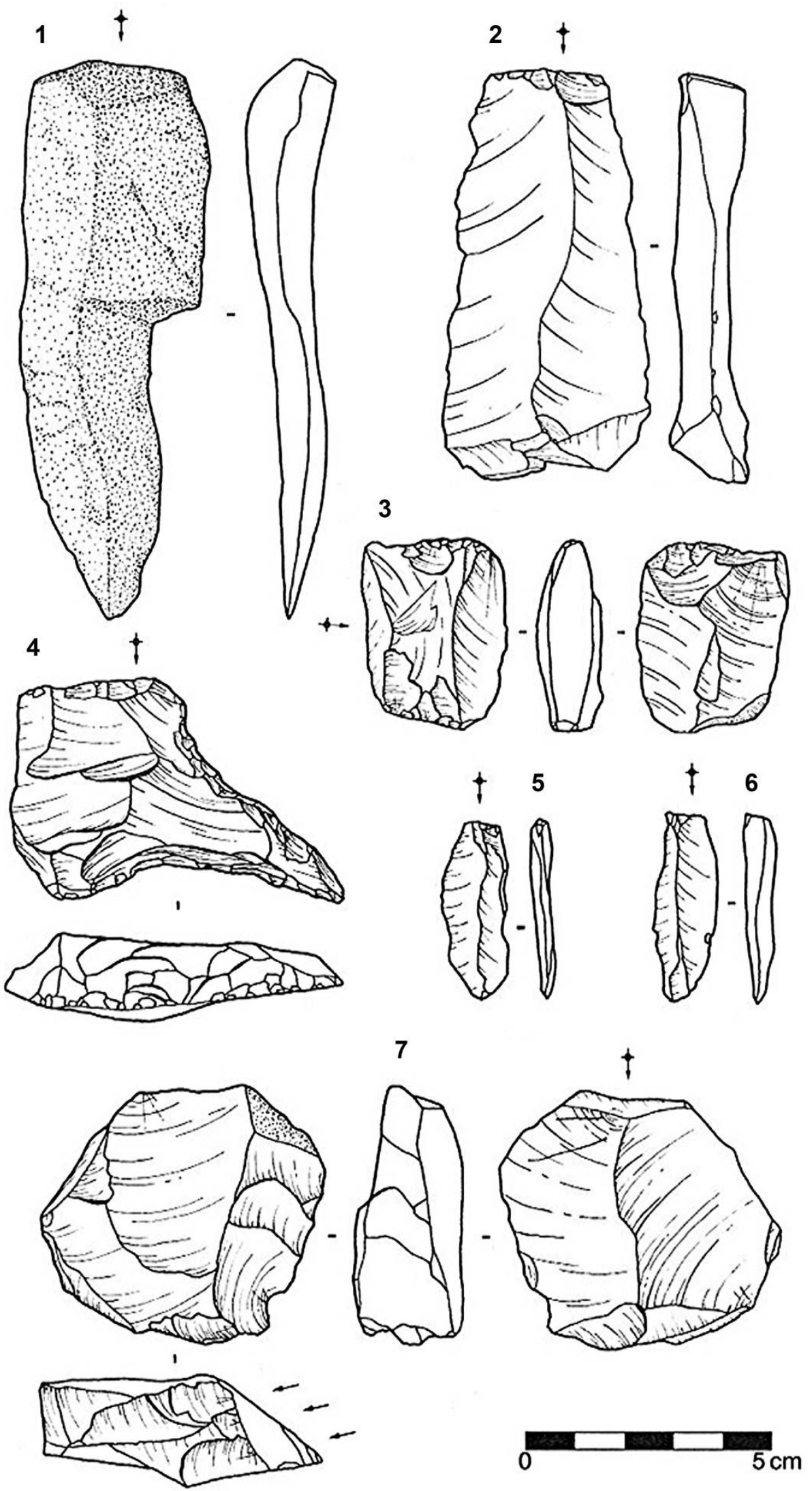

Figure 7. Restes lithiques de Homo floresiensis Liang Bua (secteur VII), Florès. 1, 2 et 5, 6: lame épaisse; 3: nucléus bipolaire; 4: bec perçoir; 7: nucléus pour la production de lamelles (d'après Morwood et al. 2004: 1991). Figure 7. Lithic remains of Homo floresiensis, Liang Bua (sector VII), Flores. 1, 2 and 5, 6: macroblade; 3: bipolar core; 4: perforator; 7: burin core for producing microblades (after Morwood et al. 2004: 1991). 
La première série de datations faites pour ces niveaux archéo-fossilifères proposait une fourchette chronologique entre 38000 ans et 18000 ans BP (Morwood et al. 2005), ce qui relançait le débat sur la possible coexistence de plusieurs humanités sur une petite aire géographique avec les Hommes dits « de la Solo », Java Central (Sambungmachan, Ngawi et Ngandong) datés entre environ 30000 et 80000 ans (Swisher et al. 1996; Yokoyama et al. 2008) et l'arrivée des Homo sapiens estimée dans la région entre 55000 et 35000 ans BP (Barker et al. 2001; Bowler et al. 2003; Turney et al. 2001; Roberts et al. 1990; O’Connell \& Allen 2004).

Une récente révision chronologique et stratigraphique de l'ensemble de ces niveaux replacerait l'Homme de Flores et ses industries entre 100000 et 60000 ans avec une dissociation entre les restes humains et un autre assemblage lithique désormais daté de 190000 BP (Sutikna et al. 2016).

Le matériel répertorié dans l'unité 4a (74000-61000 ans BP) et l'unité 9, atteste d'un événement technologique particulier avec des produits «laminaires ». Ces derniers relèvent d'un détachement «burinant » à partir d'un bord d'éclat-nucléus (flake blank cores d'après Moore et al. 2009: 506) dans le cadre d'une phase initiale de mise en forme dans la chaîne opératoire. Si certaines de ces pièces sont en effet allongées ou dites «laminaires » et apparaissent significativement dans l'assemblage, la grande majorité du matériel est représentée par des éclats. Nous ne nous trouvons pas dans le cas d'une chaîne opératoire laminaire stricto sensu.

\section{Les productions lithiques de l'Homme moderne en Indonésie}

\subsection{Les industries lithiques de Java}

Excepté les industries sur petits éclats en obsidienne d’époque prénéolithique (Holocène) découvertes dans les nombreux sites du bassin de Bandung comme la grotte de Pawon comprise entre 9500 et 5500 ans BP (Chia et al. 2008; Spriggs et al. 2011), les plus nombreuses, les plus originales et les plus étudiées sont pour l'essentiel les industries de la partie Est de Java. Notamment celles mises au jour dans les Montagnes du Sud (Gunung Sewu en Javanais ou mille collines) où pointent le plus grand nombre de sites en grotte et abri (Simanjuntak 2002; 2006; Forestier 2007b) (Figure 8).

\subsubsection{Le Sampungien : faciès à pointes de Java Est}

Découvert et inventé suite à de nombreux travaux en grotte dans les années 1930 par Van Heekeren (Van Heekeren 1972), le Sampungien se présente comme un faciès à pointes bifaciales à base concave et convexe mis en évidence dans la grotte de Gua Lawa près de Sampung dans la région de Ponorogo non loin du volcan Lawu (Figure 8). Le Sampungien fait partie de ces faciès flous devenus des fossiles directeurs à cause de leur ressemblance avec des types d'outils européens (Allchin 1966; Hooijer 1969; Van Heekeren 1972). Découvert dans un contexte stratigraphique ambigu et toujours mal daté, les pointes de Sampung (Figure 9) ont été classées comme un faciès " mésolithique indonésien » à la transition avec le Néolithique contemporain de l'Holocène ancien-moyen. S’il est encore difficile de positionner avec précision ce faciès en tant que jalon chrono-culturel, il a longtemps été chronologiquement assimilé sans démonstration à un autre faciès à pointe, le Toalien (voir infra, île de Sulawesi) (Allen 1991).

Les pointes de Sampung ont été vraisemblablement façonnées à la percussion à la pierre tendre à partir d'éclats allongés ou de plaquettes de chert local d'assez bonne qualité (rijang en javanais) et se trouvent associées à un outillage osseux très diversifié (spatule, perçoir, pointe, etc.) (Van Heekeren 1972; Forestier 2007b). Même si l'on ne connaît pas encore de 
site possédant une archéo-séquence fiable avec ce type de pièces, leur aire de répartition semble circonscrite à la partie Est de Java. Ces pointes ne trouvent d'équivalent que plus au Sud dans la région de Punung où elles ont été découvertes en surface sur des terrasses. Même si elles sont également triangulaires, bifaciales à base concave, les pointes dites de Punung sont globalement de plus grande dimension, plus épaisses et plus soignées.

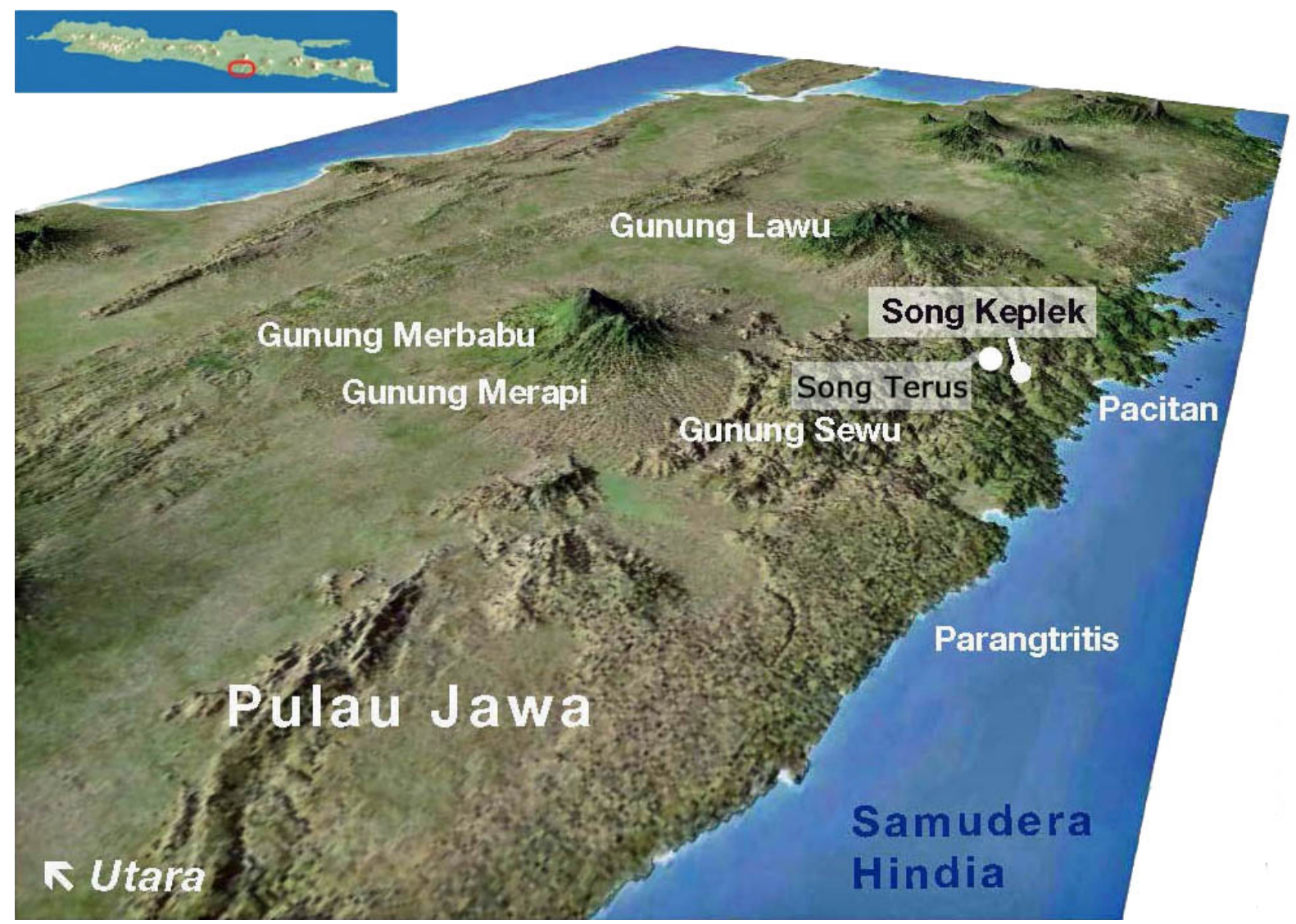

Figure 8. Vue de la partie Est de Java, Indonésie avec la localisation des deux sites de référence Song Keplek et Song Terus. (d'après Forestier 2007b; DAO: Enrique).

Figure 8. View of the East part of Java Indonesia with the location of Song Keplek and Song Terus. (after Forestier 2007b ; CAD: Enrique).

La datation et le contexte archéologique de ces pointes bifaciales (Sampung et Punung) restent pour le moment une inconnue dans la chronologie préhistorique indonésienne, tout comme les autres artefacts qui leur ont été associés (petit outillage, microlithe géométrique, outils en os, etc.).

\subsubsection{Le faciès de Keplek : faciès à outils sur éclats de Java Est}

Le site en grotte de Song Keplek a permis à la fin des années 1990 de mettre en évidence un faciès sur éclats et outils sur éclats associés à une industrie osseuse riche en spatules et en pointes (Figure 10) correspondant à la transition Pléistocène-Holocène. Song Keplek est une grotte nichée dans les montagnes du Sud de Java (Figure 8) qui a la particularité d'avoir livré un atelier de taille avec des milliers de pièces de silex dans la séquence datée entre 8000 et 5000 ans BP, au sein d'une colonne stratigraphique de 6 mètres s'échelonnant de 24420 \pm 1000 ans BP à $1940 \pm 120$ ans BP (Simanjuntak 2002; Forestier 2007b).

La méthode de taille mise en œuvre à Song Keplek (un débitage orthogonal) se retrouve dans un grand nombre de sites javanais de la même période mais aussi dans l'ensemble de l'archipel (Forestier 1999; 2000). 

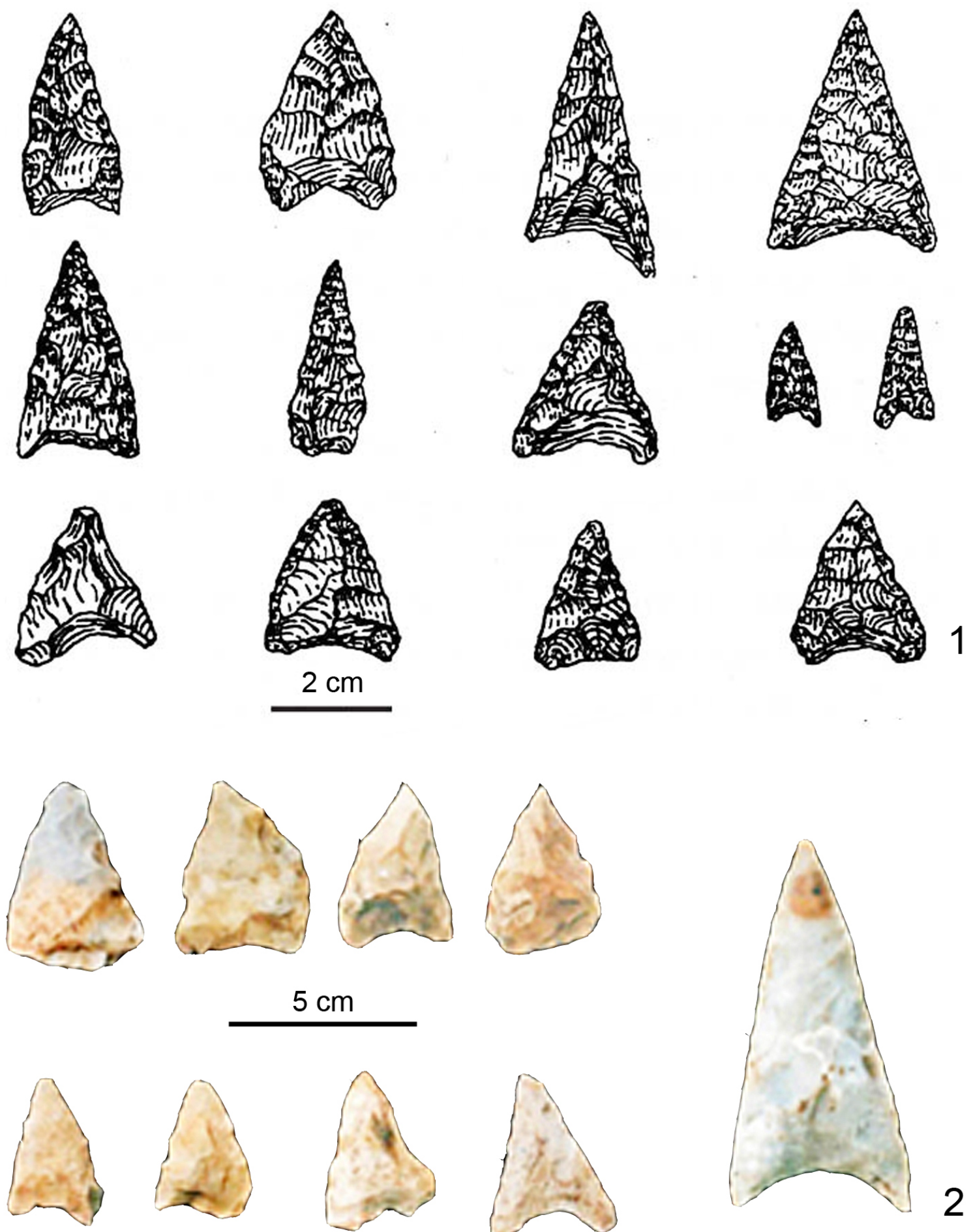

$5 \mathrm{~cm}$
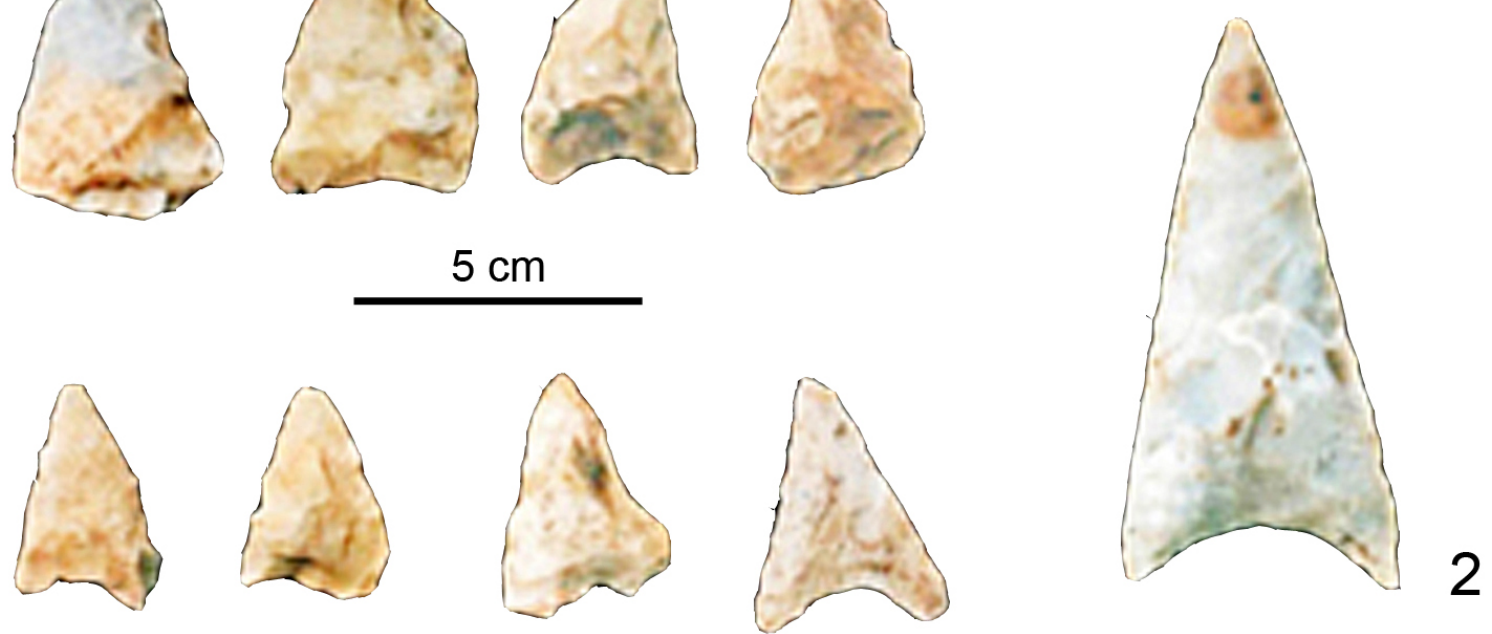

Figure 9. Pointes bifaciales à base concave de l’Est de Java: 1: Gua Lawa, Sampung (d'après Allchin 1966); 2: Punung, Gunung Sewu, (Forestier).

Figure 9. Bifacial arrowheads with concave base from East Java : 1: Gua Lawa, Sampung (after Allchin 1966); 2: Punung, Gunung Sewu, (Forestier). 

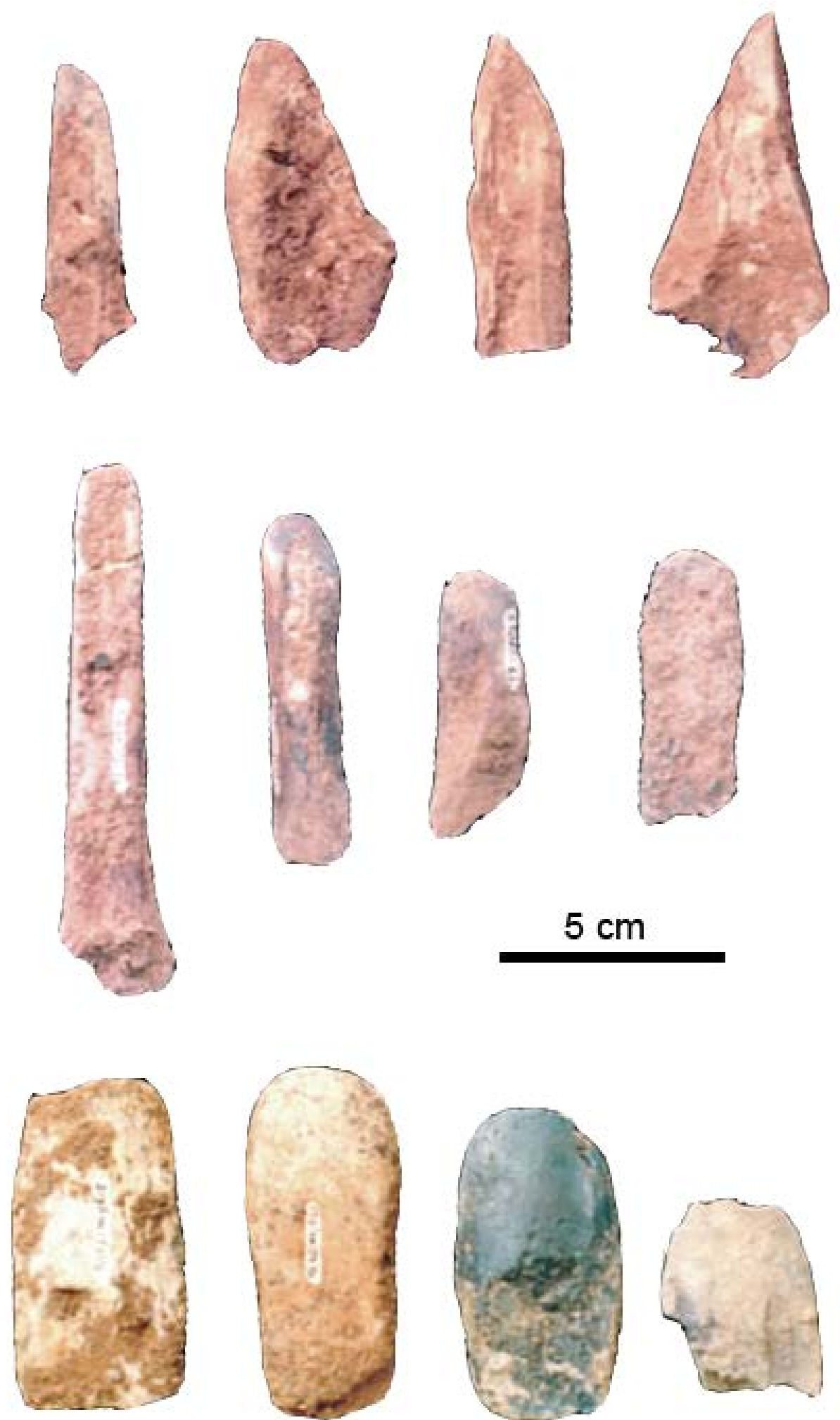

Figure 10. Industrie osseuse de Song Keplek, Java Est. (d’après Forestier 2007b). Figure 10. Bone tools of Song Keplek, East Java. (after Forestier 2007b).

Le schéma opératoire de Song Keplek est dit "élémentaire » car non Levallois et non laminaire même si les supports tendent vers l'allongement. Il s'identifie au travers d'un débitage préférentiellement d'orientation orthogonale de type SSDA tel que défini par Forestier (1993; 2000; 2007b). La répétition de ce procédé (ou algorithme) vise, dans ce cas précis, à produire des supports généralement quadrangulaires fortement corticaux $(>70 \%)$ 
dont la longueur varie en fonction de la morphologie du bloc de départ et de la récurrence de l'algorithme (Figure 11).
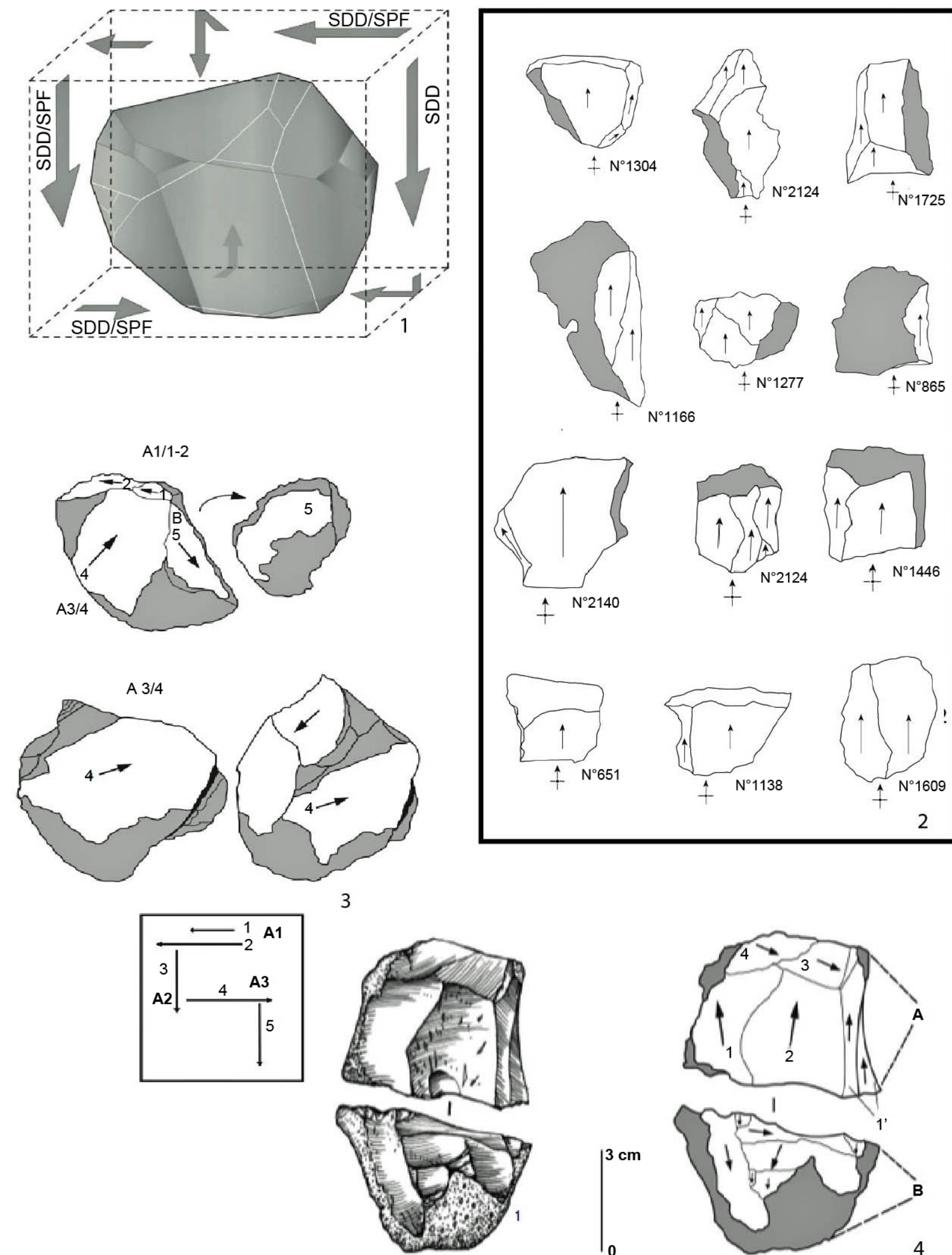

Figure 11. Principe d'application du débitage orthogonal (1), nucléus remonté (2), éclats (3) et nucléus (4), Song Keplek, Java Est. (d’après Forestier 2007b)

Figure 11. Orthogonal debitage (1), refitting core (2) flakes (3) and core (4), Song Keplek, East Java. (after Forestier 2007b). 
Lorsque les supports sont allongés et corticaux, les outils confectionnés relèvent d'une typologie très « moustéroïde " avec des couteaux à dos naturel, des racloirs, des grattoirs, des becs, des perçoirs, des coches, des limaces, et des denticulés (Figure 12).
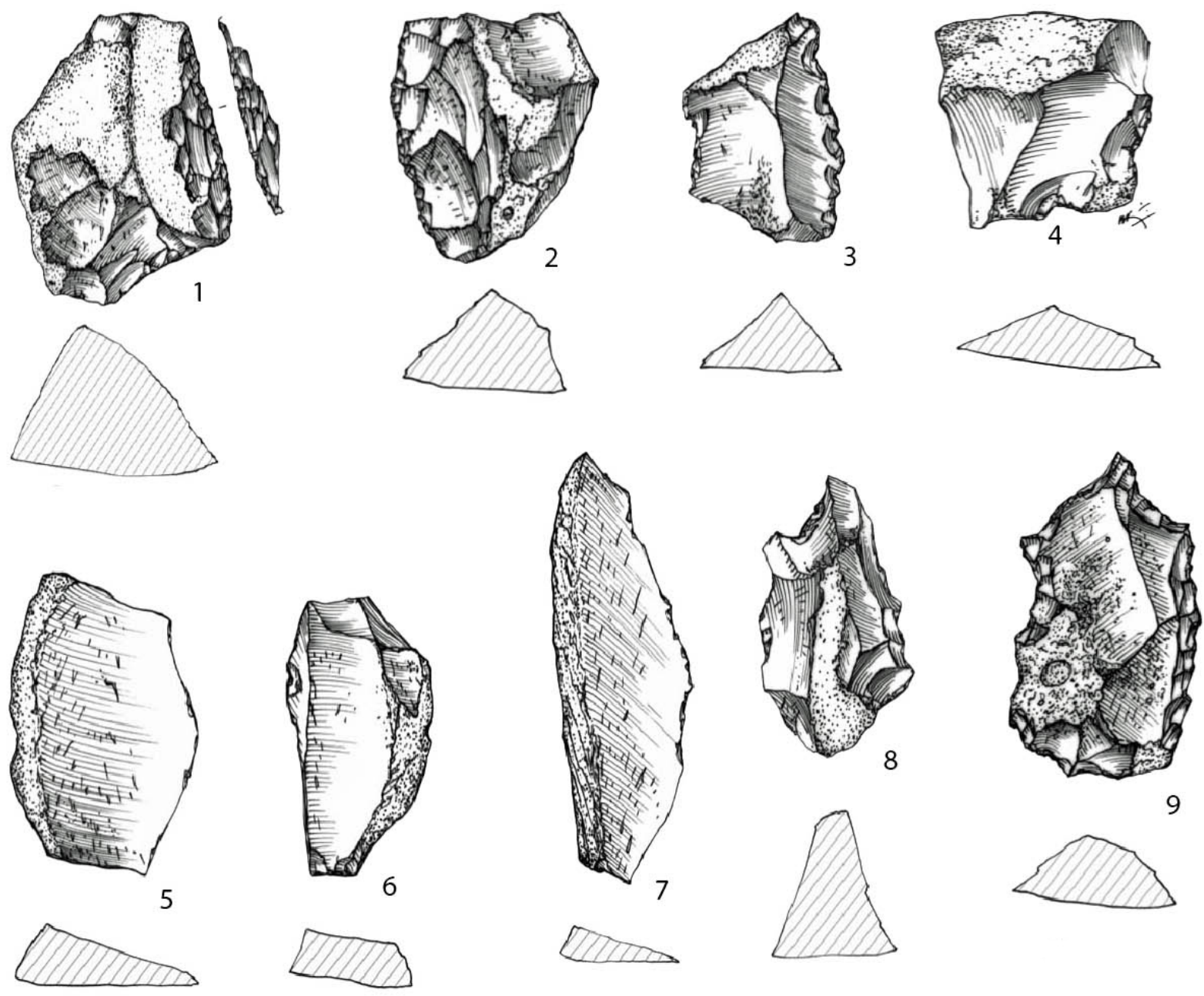

9

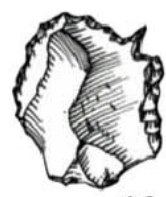

10
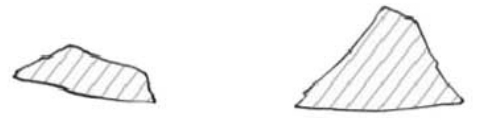

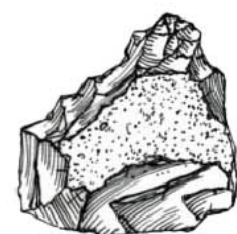

12

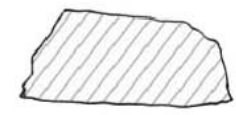

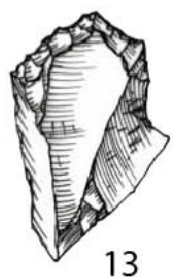

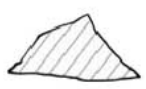

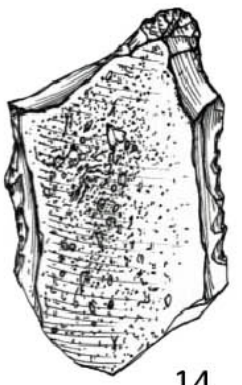

14

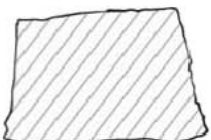

Figure 12. Outils sur éclats de Song Keplek, Java Est. 1, 2: racloir; 3: denticulé; 4: coche; 5, 6, 7: couteau à dos naturel; 8: limace; 9: bec (composite racloir latéral); 10, 11: perçoir; 12, 13, 14: grattoir. (d'après Forestier 2007b).

Figure 12. Flake tools of Song Keplek, East Java. 1, 2: scraper; 3: denticulated; 4: notch; 5, 6, 7: natural back knive; 8: limace; 9, 11: drill (perforator); 12, 13, 14: end-scraper. (after Forestier 2007b).

Cette méthode de taille ou SSDA (Forestier 1993) a la particularité de ne pas faire intervenir de phase d'initialisation (mise en forme de nucléus) ce qui explique d'une part la 
récurrence des types d'éclats et d'autre part, la forte variabilité des formes de nucléus à tous les stades d'exploitation jusqu'au nucléus épuisé ou multiplatform cores: une logique organisatrice du débitage répondant à une invariabilité (éclats) sous transformation (nucléus) (Figure 13).

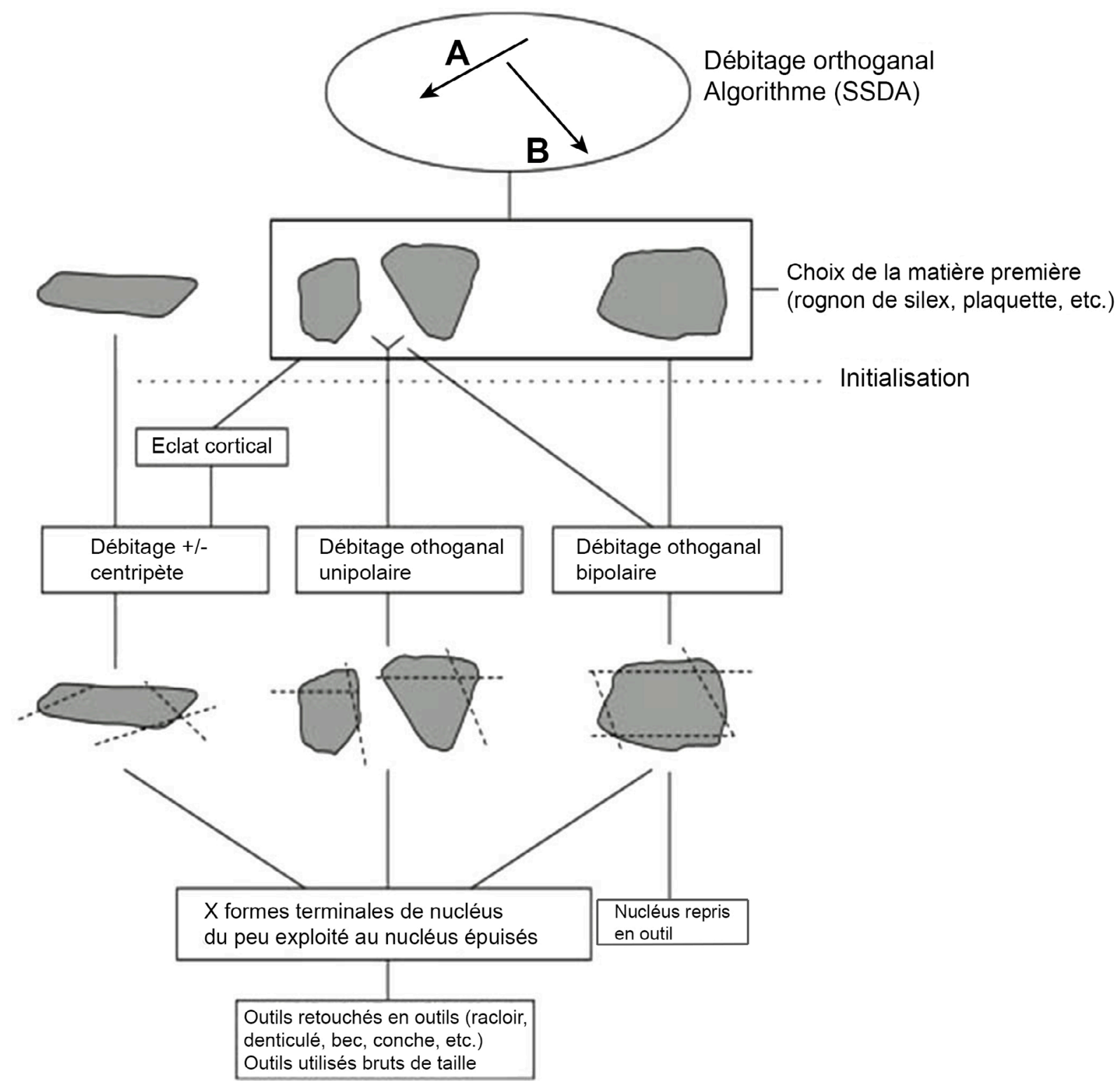

Figure 13. Schéma global du débitage orthogonal de Song Keplek, Java Est. (d’après Forestier 2007b).

Figure 13. Global sketch of the orthogonal debitage sequence of Song Keplek, East Java. (after Forestier 2007b).

Ce type de débitage souvent qualifié d' «orthogonal » produit plusieurs types d'éclats caractéristiques (Figure 11) selon la technique de la percussion directe à la pierre dure : éclat d'entame, éclat plus ou moins corticaux à négatifs d'enlèvements parallèles unipolaires et orthogonaux, éclats à dos corticaux, éclats allongés corticaux ( \pm opportunistes).

L'application directe de l'algorithme s'impose comme une hiérarchisation autorégulée dès le détachement des premiers enlèvements jusqu'à l'arrêt du débitage. L'orthogonalité recherchée se surimpose à la forme du bloc de départ selon Surface de Plan de Frappe (SPF) versus Surface de Débitage (SDD), tout en lui conférant le statut de nucléus. L'algorithme, dans ce cas, devient à la fois structure, structuré et structurant. Autrement dit, une surface de débitage (épisode-négatifs d'enlèvements) devient à son tour, si l'angle est maintenu $\left(\leq 90^{\circ}\right)$, 
une possible surface de plan de frappe pour une nouvelle série d'éclats ( 1 à 3 en moyenne) et ainsi de suite jusqu'à exhaustion du nucléus. Ce mode de débitage d'éclats est très certainement la méthode de taille la plus répandue dans l'archipel pour produire des éclats (courts et allongés) et ce, quelle que soit la période du Paléolithique.

Un autre site important des Montagnes du Sud de Java situé non loin du précédent (Figure 8) est le site Song Terus qui présente une archéo-séquence exceptionnelle de près de 15 mètres de puissance devenue une référence pour les enregistrements archéologiques de longue durée en contexte karstique en Asie du Sud-Est. On y retrouve une distribution d'industries lithiques s'échelonnant du Pléistocène moyen dès $341000 \pm 51$ ans BP jusqu'au milieu de l'Holocène il y a 5500 ans BP (Sémah et al. 2004a; 2004b; Hameau et al. 2007). Aucun niveau néolithique n’y est présent. Trois niveaux archéologiques y ont été définis. On y retrouve, à quelques variantes près, la même méthode de taille orthogonale et des multiplatform cores qu'à Song Keplek.

Ces trois niveaux sont, du plus récent au plus ancien :

- La couche holocène dite de Keplek comprise entre 10000 et 5000 ans BP qui présente une industrie sur éclats similaire à celle rencontrée à Song Keplek. Ce niveau supérieur est nommé « couche Keplek » par Forestier (2007b) et Borel (2012).

- La couche intermédiaire dite de Tabuhan comprise entre 80000 et 30000 ans BP dans laquelle aucun outil en pierre caractéristique sur un plan typologique n’a été trouvé (Communication personnelle avec F. Sémah en 2017).

- la couche ancienne de Terus, datée du Pléistocène moyen final au Pléistocène supérieur final, montre une production d'éclats et d'outils sur éclats relevant d'un mode de débitage orthogonal. La lecture des négatifs d'enlèvement majoritairement unidirectionnels et orthogonaux sur la face supérieure le confirme (Tiauzon 2011) tout en affichant une grande homogénéité métrique et des nucléus à morphologie et plan de frappe multiples. L'exploitation continue d'un chert récupéré dans l'environnement proche de la grotte et l'uniformité apparente du mode de débitage sans variation ni rupture nette, donnent un panorama technologique stable sans vraiment de changement radical de méthode de taille entre $341000 \pm 51$ ans BP jusqu'au niveau supérieur daté bio-chronologiquement par la faune de Punung du MIS5.

D'un point de vue diachronique, sur plus de 300000 ans d'enregistrement archéologique, nous n'observons pas de changement radical dans les méthodes et les techniques de taille. Un même mode de débitage orthogonal (SSDA) prédomine toute le long de l'archéo-séquence (i.e. sur plusieurs mètres et sur plusieurs dizaines de milliers d'années). En revanche, les données environnementales indiquent des changements (Sémah et al. 2002; Sémah et al. 2004a; 2004b) et les données archéozoologiques montrent des stratégies différentielles de subsistance au moins durant le Pléistocène supérieur jusqu'au début de l'Holocène (Ingicco 2012; Amano et al. 2016).

Hormis peut-être la dimension des supports-outils, le seul changement significatif majeur connu pour le matériel archéologique est l'apport d'outils en os dans le niveau Holocène (niveau Keplek) apportant ainsi une variabilité dans la composition de l'outillage (Setiagama 2006). Si l'étude des modes de production lithique ne permet pas de faire de différence notable entre les trois jalons culturels, d'autres approches comme le degré d'investissement par la retouche des bords ou la morphométrie géométrique-fonctionnelle permettront, peutêtre, de mettre en évidence des caractères discrets et discriminants entre tel ou tel type de support-outil le long de la séquence (étude en cours par A. Tiauzon dans le cadre d'une thèse de doctorat). 


\subsection{Les industries lithiques de Sumatra : suite et fin du peuplement hoabinhien}

L'île de Sumatra $\left(450000 \mathrm{~km}^{2}\right)$ s'étire sur près de $1500 \mathrm{~km}$ de part et d'autre de l'équateur et s'impose définitivement à l'Holocène lors de la remontée marine comme la porte d'entrée de l'Archipel. Largement moins prospectée et fouillée pour des raisons d'impénétrabilité environnementales et d'inhospitalité notoire, elle a néanmoins attiré l'attention de chercheurs comme Eugène Dubois dès son arrivée en Indonésie à la fin du XIX $^{\text {ème }}$ siècle (karst de Payakumbuh, Sumatra Ouest, région de Padang). Par la suite d'autres chercheurs ont mis en évidence le potentiel de l'île avec notamment la découverte de sites de plein air hoabinhiens et des assemblages d'outils sur éclats en obsidienne (Figure 14) (Brandt 1976; Glover 1979a; Soejono 1984a; McKinnon 1990; Simanjuntak 1995; Forestier 2007a).

Deux types de chaînes opératoires sont présents à Sumatra dans des sites, principalement en grottes et abris, dont les occupations remontent à la fin du Pléistocène supérieur ou au début de l'Holocène : une chaîne de débitage d'éclats sur du silex ou de l'obsidienne et une autre, de façonnage de galets qui se rapporte à du Hoabinhien.

Tout d'abord une chaîne opératoire de débitage qui a été utilisée fréquemment sur de l'obsidienne un peu partout sur l'île comme dans la grotte de Tianko Panjang, Province de Jambi (Figure 15) dont le niveau archéologique avait été daté d'environ 10000 ans BP (Bellwood 1997; Bronson \& Asmar 1975; Bronson \& Wisseman 1974). La méthode de taille est assez simple et relève d'un débitage orthogonal sur des petits blocs qui, après exploitation, aboutissent en général à des nucléus assez informes voire polyédriques de petite dimension.

La production de supports est marquée par de nombreux éclats d'entame et autre éclats semi-corticaux souvent plus larges que longs, retouchés en racloir, coche et denticulé. Il n'existe pas de façonnage stricto sensu de pointe dans cette matière première ni dans un silex local. On retrouve ce même débitage dans de nombreux autres sites d'époque Holocène en grotte dans le massif karstique de Baturaja (Padang Bindu), plus au Sud, qui renferment des niveaux d'occupation remontant au pré-Néolithique (3000-4500 ans BP) et au Néolithique (2000 ans BP) comme celle de Pondok Silabe1 (SLB1, Figure 16) (Simanjuntak \& Forestier 2004). Le débitage est le plus souvent orthogonal (nucléus prismatique et polyédrique) et l'approvisionnement en matières premières (silex ou obsidienne) provient de sources locales.

L'autre chaîne opératoire rencontrée à Sumatra est de nature hoabinhienne, c'est à dire réalisée à partir d'une chaîne opératoire de façonnage unifacial sur galets plats oblongs de section plano-convexe. Le Hoabinhien de Sumatra est connu pour être côtier. Il se différencie du continent en associant au façonnage de galets (unifaces) une production d'éclats tirés d'un débitage orthogonal parfois même discoïde.

Cette diversité des modes opératoires commencerait, semble-t-il, dans le Sud de la Thaïlande, non loin de la frontière avec la Malaisie (isthme de Kra), avec l'apparition d'outils nouveaux sur éclats épais comme les limaces découvertes en abondance à Moh Khiew (Auetrakulvit et al. 2012). On retrouve ces industries sur galets préférentiellement dans le Nord-Est de Sumatra sous la forme d'amas coquilliers remontant au début de l'Holocène (Glover 1979a; McKinnon 1990) et plus rarement en grotte comme à Tögi Ndrawa sur l'île de Nias dont la séquence couvre la totalité de l'Holocène (i.e. de 9000 ans BP à 900 ans BP) (Forestier et al. 2005b; Forestier 2007a) (Figure 17).

La grotte de Gua Pandan, dans le massif de Padang Bindu à Sumatra Sud, dont la couche archéologique est datée entre 9000 et 6500 ans BP, présente des outils unifaciaux lourds façonnés sur galets de calcaire, des limaces et un micro-outillage sur éclat en chert et en jaspe rouge (Forestier et al. 2006) (Figures 18 et 19). 


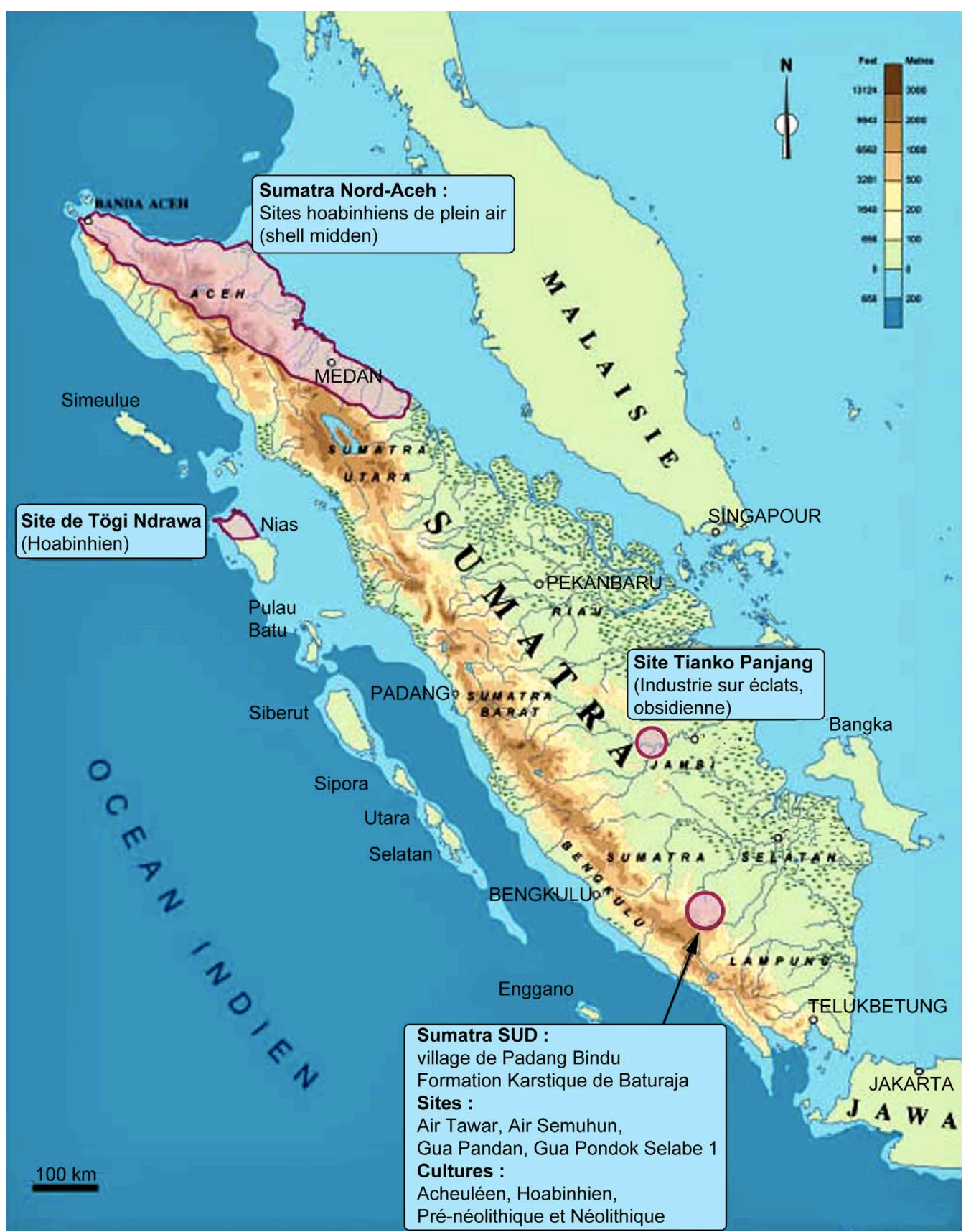

Figure 14. Carte de l'île de Sumatra et des principaux sites préhistoriques (Forestier 2007a)(DAO : Laurence Billault).

Figure 14. Sumatra island map and the major prehistoric sites. (Forestier 2007a)(CAD: Laurence Billault).
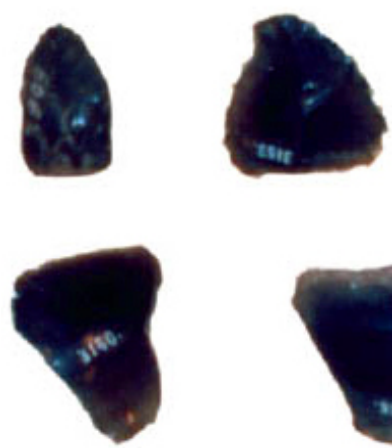
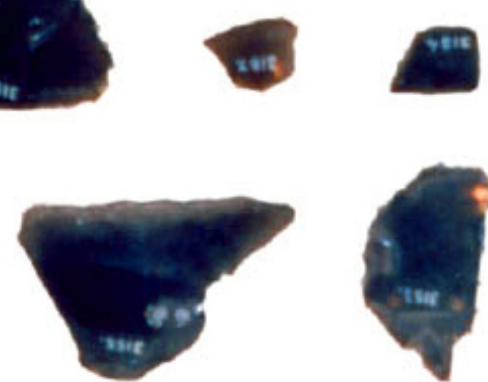
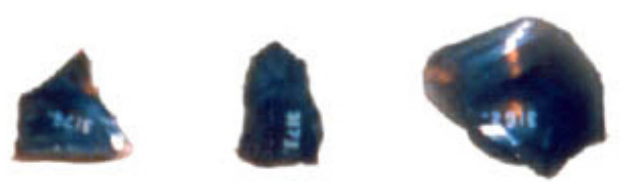

\section{$1 \mathrm{~cm}$}
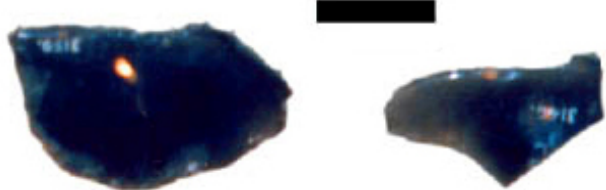

Figure 15. Eclats sur obsidienne (1) et silex (3), grotte de Tianko Panjang (2), Sumatra. (Forestier). Figure 15. Obsidian (1) and chert flake industry (3), Tianko Panjang cave (2), Sumatra. (Forestier). 

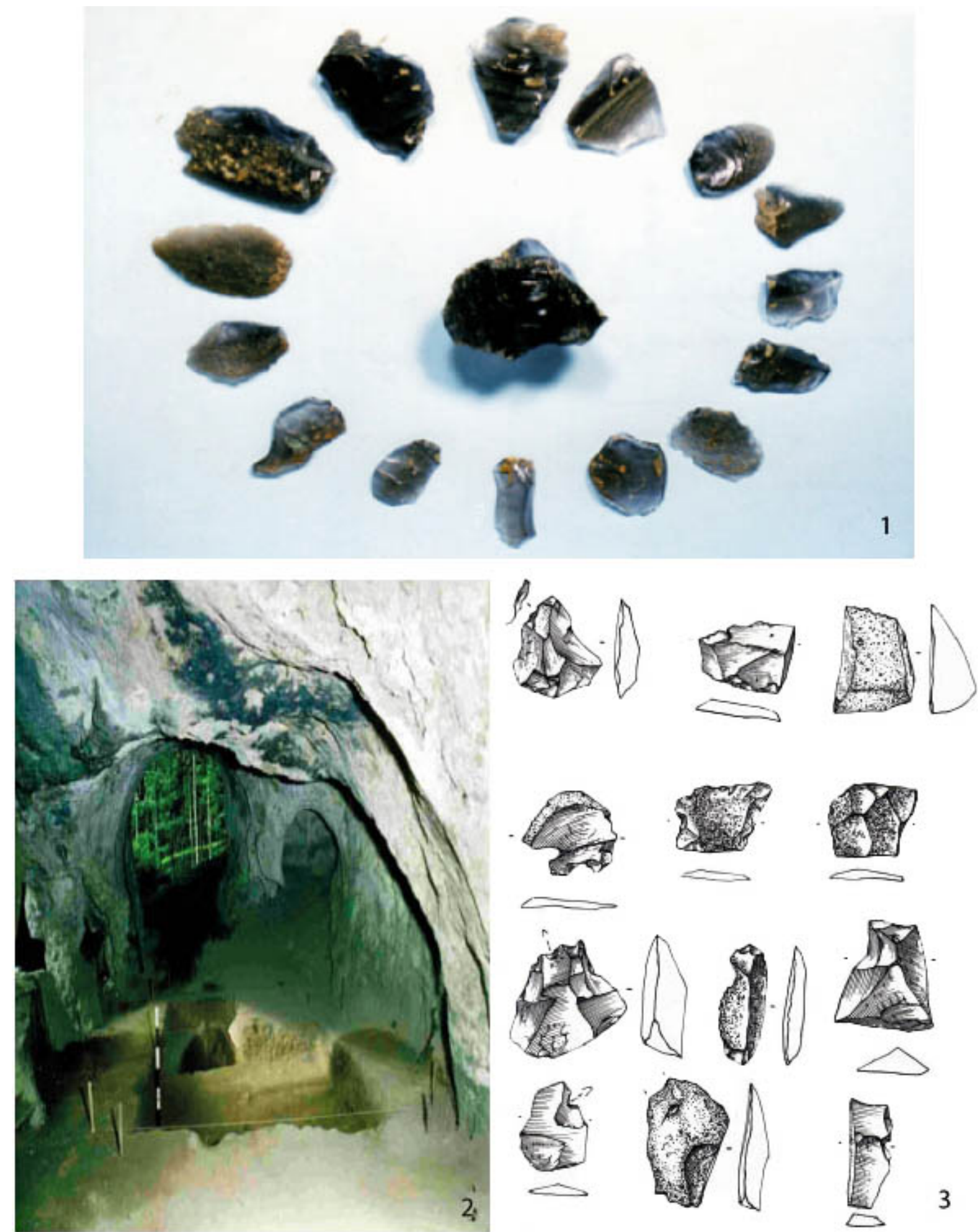

Figure 16. Eclats de silex et d’obsidienne du niveau prénéolithique, SLB1, Sumatra Sud. (Forestier).

Figure 16. Chert and obsidian flakes from preneolithic level, SLB1, South Sumatra. (Forestier). 

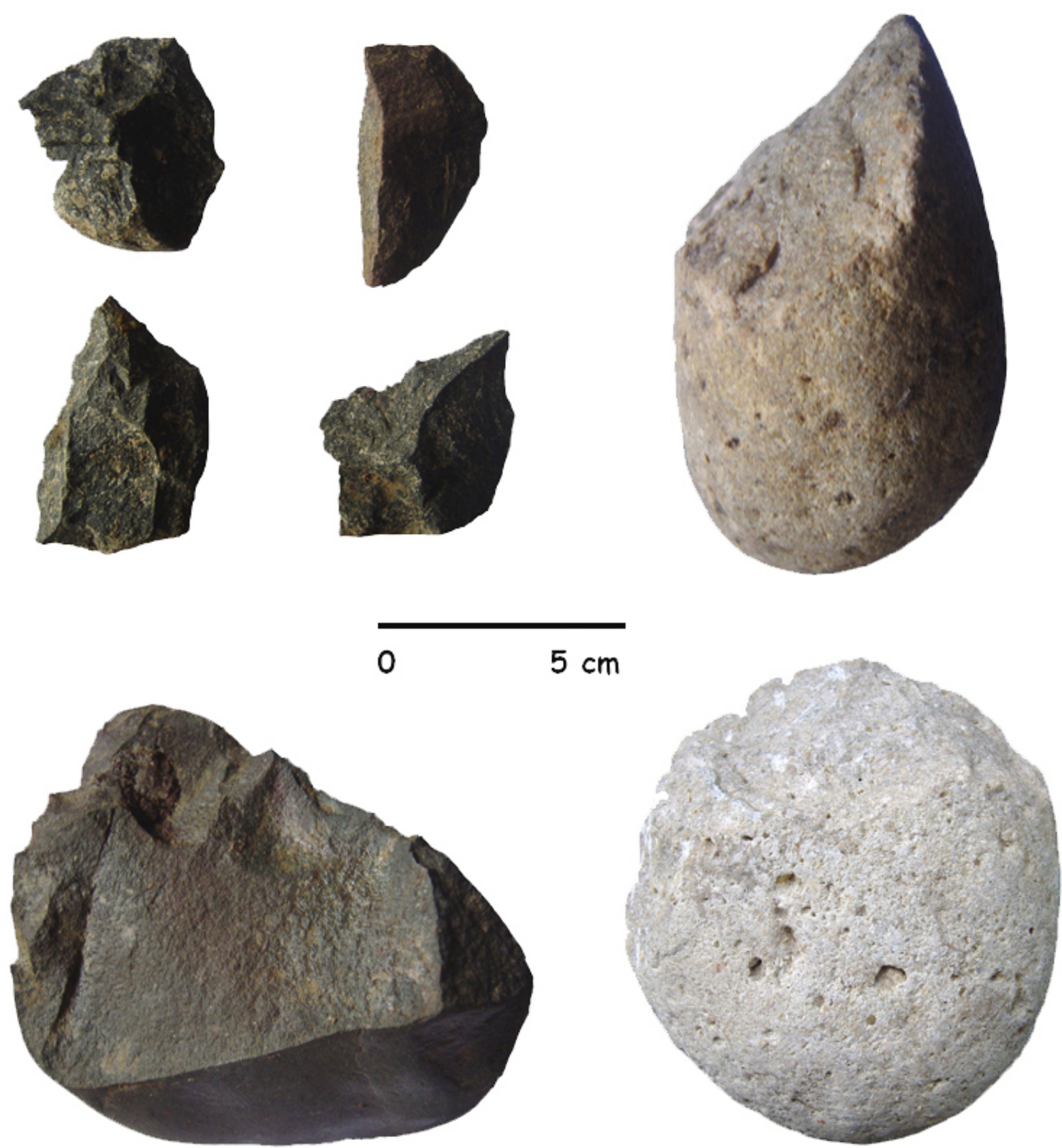

Figure 17. Assemblage hoabinhien du site de Tögi Ndrawa, île de Nias, Sumatra Nord. (Forestier) Figure 17. Hoabinhian assemblage of Tögi Ndrawa site, Nias Island, North Sumatra. (Forestier)

Il reste encore beaucoup à découvrir de cette île qui affiche une préhistoire complexe et une variété d'industries à la croisée d'influences continentales (Hoabinhien) et insulaires (éclats, pointes ?). Un grand nombre d'assemblages lithiques est mixte (débitage et façonnage de galets), marqué par des choix nouveaux de matières premières (obsidienne, silex, jaspe, etc.) mais aussi par l'apparition de nouvelles formes d'outils sur éclat comme par exemple les limaces. Si la grande île de Sumatra peut être perçue dans sa partie Nord comme affichant une relative homogénéité industrielle héritée des influences hoabinhiennes (Malaisie, Thaïlande, Cambodge) avec notamment une tradition de façonnage de galets; elle se place, d'une certaine façon, déjà comme un creuset annonçant l'hétérogénéité caractéristique de Java et des autres îles du plus grand archipel du monde (Simanjuntak 1995; Forestier et al. 2010). 

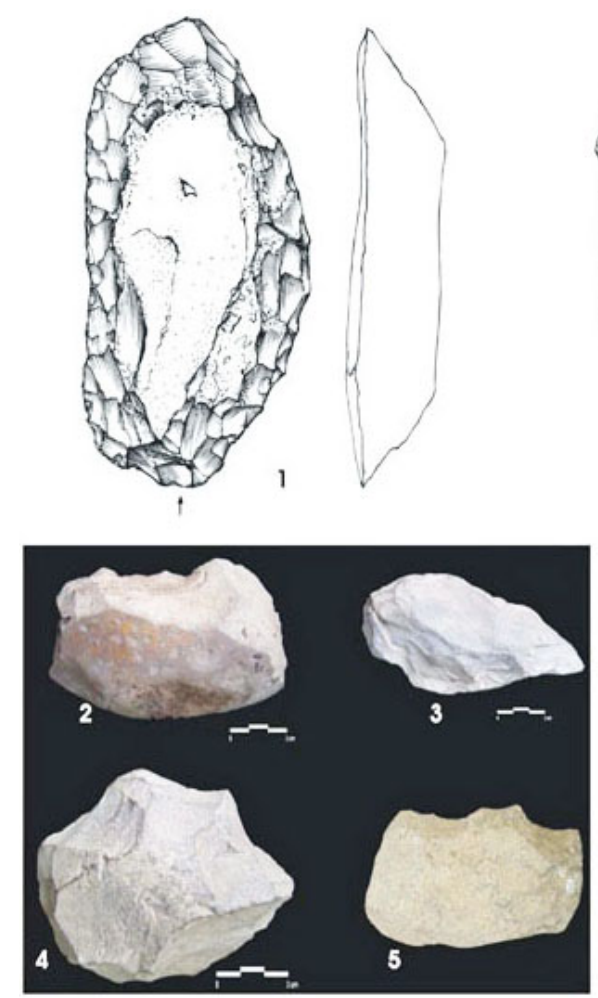

Figure 18. Outils du niveau hoabinhien de la grotte de Gua Pandan, Sumatra Sud. 1 à 5 et 11: macro-outils; 1 , 11: uniface; 3: limace; 6: pointe pseudo-Levallois retouchée; 7 à 10: outils sur éclats. (Forestier).

Figure 18. Tools of the Hoabinhian level of Gua Pandan cave, South Sumatra. 1 to 5 and 11: macro tools; 1, 11: uniface; 3: limace; 6: retouched pseudo-Levallois point; 7 to 10: flake tools. (Forestier)
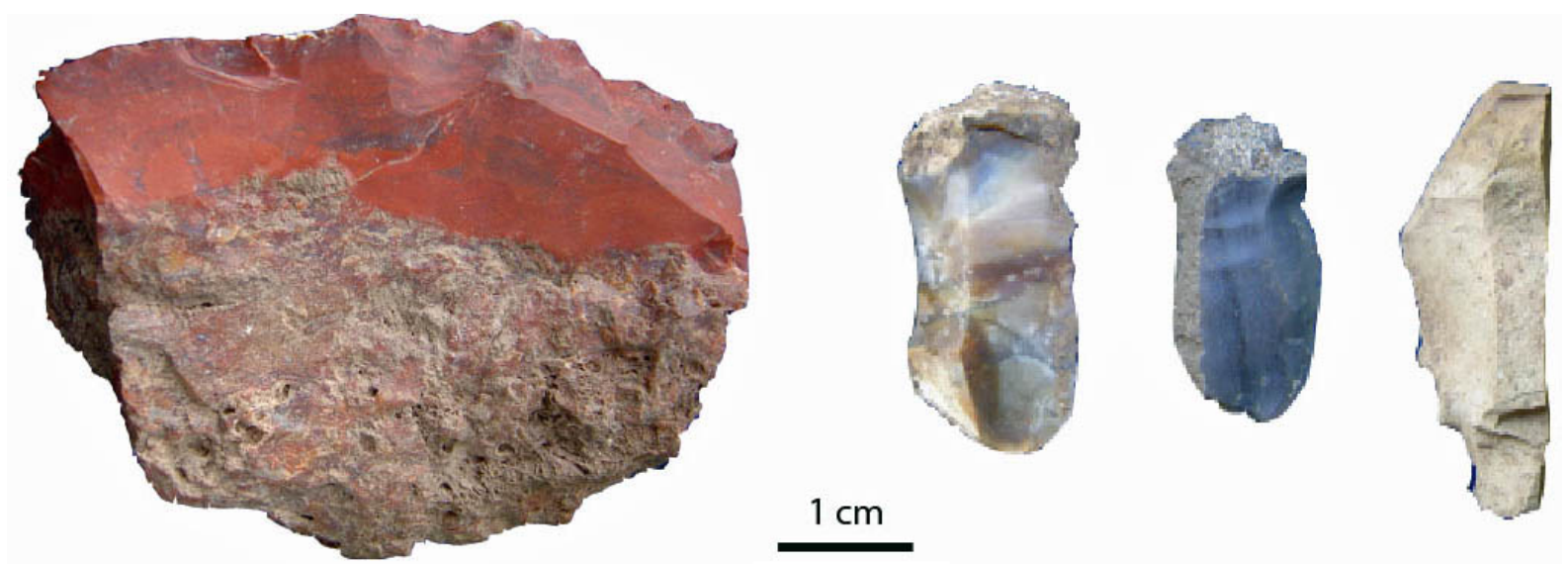

Figure 19. Denticulé sur éclat (jaspe rouge) et trois éclats laminaires en silex, Gua Pandan, Sumatra Sud. (Forestier).

Figure 19. Denticulated on flake (red jasper) and three laminar flakes on chert, Gua Pandan, South Sumatra. (Forestier).

\subsection{Les industries lithiques de Bornéo : connues et inconnues}

\subsubsection{Sarawak et Sabah}

Si l'on se réfère aux connaissances relatives aux systèmes de production lithique d'Asie du Sud-Est, l'île de Bornéo $\left(730000 \mathrm{~km}^{2}\right)$ apparaît comme une sorte de Terra incognita à l'exception de quelques sites dans sa partie malaise (Sarawak, Sabah) et d'autres dans la partie indonésienne (Kalimantan Ouest, Est et Sud). Compte tenu de l'immensité et de la difficulté d’y travailler, la préhistoire de Bornéo n’en est qu’à son commencement. 
La partie malaise a livré plusieurs sites de référence comme Gua Sireh, Lubang Angin (Datan 1993; Datan \& Bellwood 1991), et les fameuses grottes de Niah à Sarawak (Harrisson 1957; 1959; Barker 2013), les sites des massifs de Madai et Baturong et de Bukit Tengkorak à Sabah (Bellwood 1984; Bellwood \& Koon 1989) (Figure 20).

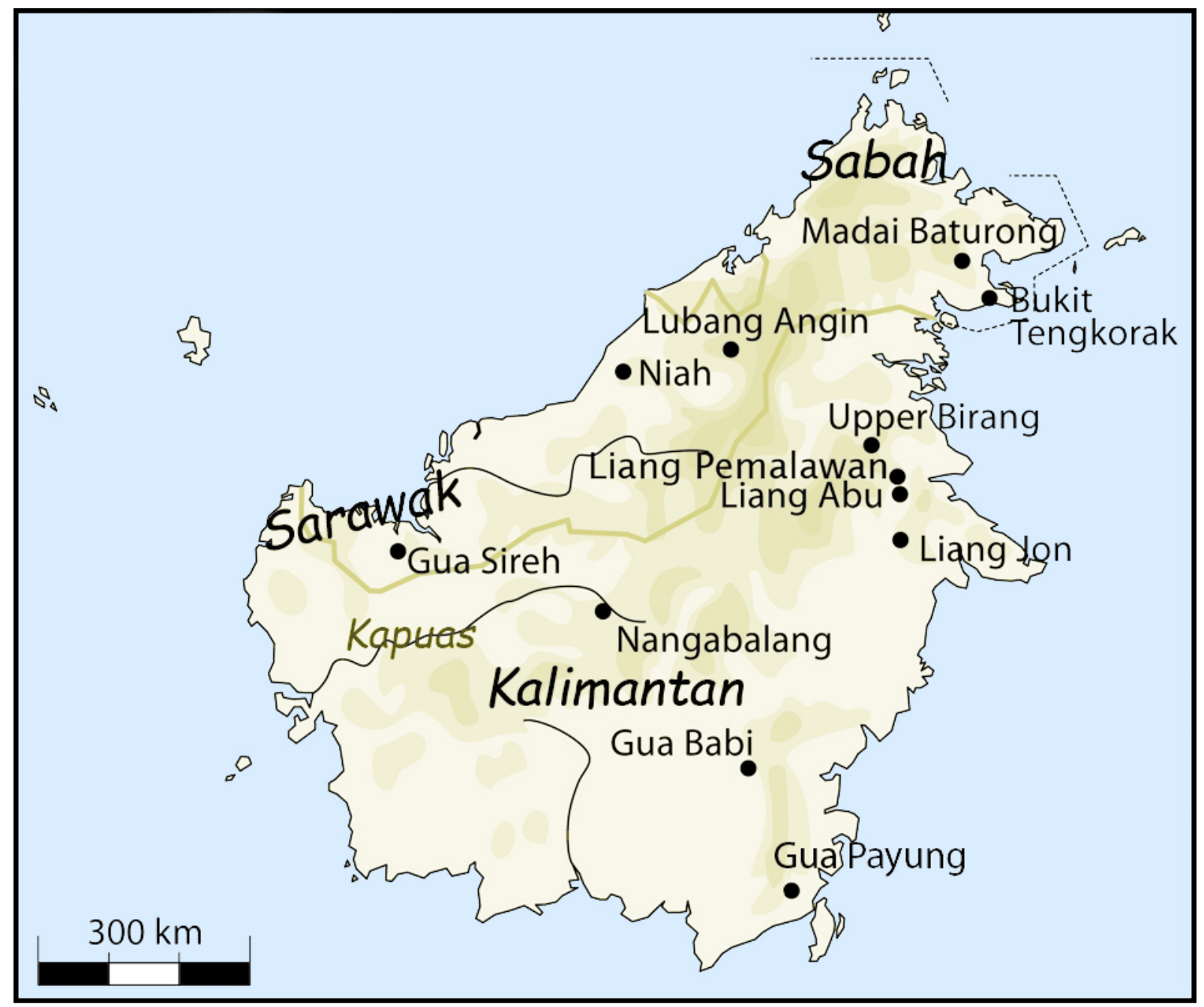

Figure 20. Carte des principaux sites de Bornéo. (d'après Grenet et al. 2016).

Figure 20. Map of the major prehistoric sites of Borneo. (after Grenet et al. 2016).

Paradoxalement l'aire indonésienne (Kalimantan) qui représente la plus grande partie de Bornéo reste peu exploitée archéologiquement si ce n’est par quelques sites ayant livré des stratigraphies détaillées avec des datations fiables. Concernant les sites de la partie malaise, il en existe encore moins qui peuvent servir de référence pour discuter des systèmes de production lithique pléistocènes holocènes.

Les principaux sites de Kalimantan sont localisés (Figure 20): (1) sur le cours supérieur de la rivière Kapuas (Kalimantan Ouest), comme Nanga Balang (Soejono 1991); (2) Gua Babi (Widianto 1997) and Gua Payung (Fajari \& Kusmartono 2013) dans le massif de Meratus (Kalimantan Sud); (3) les sites de Kimanis, Liang Gobel and Lubang Payau dans la partie amont de la rivière Birang (Arifin \& Delanghe 2004), dans le district de Berau (Kalimantan Est); et (4) les grottes of the Keboboh (Jatmiko et al. 2004), Gua Tengkorak (Chazine 2005), Gua Tebok, Gua Batu Adji, Gua Lungun (Gunadi 2006), Liang Jon (Chazine 2005; Chazine \& Ferrié 2008; Grenet et al. 2016) Liang Abu and Liang Pemalawan (Ricaut et al. 2011; 2012; 2013; Plutniak et al. 2014; Grenet et al. 2016). 
La grande grotte de Niah (West Mouth) présente une séquence néolithique très riche en pièces polies et matériel complet de broyage (4000-2000 ans BP) précédée de niveaux du Pléistocène final et de l'Holocène ancien (11500-8000 ans BP) où l'on observe des chaînes opératoires de production d'éclats qui relèvent d'un débitage de type orthogonal (basic core and flake reduction sequence d'après Majid 1982; Barker et al. 2011).

Le site côtier du lac Tingkayu dans le massif des Madai-Baturong à Sabah, au Nord de l'île, a livré dans un contexte de paléo-lac un assemblage original de pièces bifaciales en chert daté entre 28000 et 16000 ans BP. Il s’agit de pointes bifaciales lancéolées (lanceolate knives, small bifaces) probablement accompagnées de préformes qualifiées de large bifaces (Figure 21) (Bellwood 1983; 1984; 1988; Saidin \& Jeffrey 2014). Cette chaîne opératoire de façonnage et la qualité de la matière première indiquent des comportements et des savoir-faire nouveaux pour cette période du Paléolithique final en Indonésie et en Asie du Sud-Est dans sa globalité. Ces pièces font figure d'exception technique et d'un savoir-faire spontané dans cette zone géographique excentrée de l'archipel.

Toujours dans le massif des Baturong, l'abri de Hagop Bilo daté d'entre 18000 et 12000 ans BP (Bellwood 2007) a livré une série d'éclats en chert, allongés, plutôt épais et pouvant être appointés. Le site présente aussi une industrie sur galets, des éclats, des nucléus à plan de frappe unique ou multiple (débitage orthogonal ?) et des types de racloirs épais (Bellwood 2007: 180).

Le site de Bukit Tengkorak daté d'entre 5500 et 2000 ans BP est un site côtier localisé à proximité de Semporna (Sabah) sur un petit promontoire face à la mer des Célèbes. Des séries laminaires y ont été identifiées sur des matériaux particuliers (Figure 22) : l'agate (provenance locale) et l'obsidienne (provenance exogène lointaine). Les nucléus de petite taille sont décrits à juste titre comme prismatiques laminaires, i.e. "small prismatic or wedge-shaped blade cores » (Bellwood \& Koon 1989: 618) et les produits sont souvent comparés à ceux découverts dans des sites du Nord-Est de l’Indonésie (Sulawesi, Toalien).

Un cas similaire d'utilisation de matière première exogène est connu pour la même tranche chronologique dans une aire de transition géographique déjà presque océanienne, l'archipel des îles Talaud situé au Nord de la Wallacea entre les mers des Célèbes et des Philippines.

On note des éclats de petite dimension et de très petits éléments laminaires en obsidienne mesurant moins de $20 \mathrm{~mm}$ de longueur (Bellwood 1976). Cette obsidienne proviendrait d'une source connue de Mélanésie septentrionale, la Talasea située à près $2000 \mathrm{~km}$ à l'Est sur l'île de Nouvelle-Bretagne dans l'archipel de Bismarck (Papouasie-Nouvelle-Guinée).

Ce débitage lamino-lamellaire reste une autre exception typo-technologique et pourrait être rattaché d'après Bellwood \& Koon (1989: 619) à des répertoires technologiques néolithiques sud-chinois ou philippins. Ce débitage de lamelles n'entre pas dans le cadre technologique d'une production laminaire au sens strict du terme puisqu'il n'y a pas de mise en forme classique des nucléus (crêtes, carènes, convexités). Il semblerait que l'exploitation soit dirigée par les opportunités morphologiques et les caractéristiques physiques particulières des blocs et éclats d'agate et d'obsidienne (nucléus sur tranche d'éclat ou arête de bloc).

On peut trouver des similitudes morpho-métriques entre les produits laminaires de Bukit Tengkorak avec ceux trouvés dans d'autres sites compris entre 5500 et 2000 ans BP du NordEst de l'Indonésie, des Philippines comme à Duyong Cave sur l'île de Palawan (Fox 1970) ou à Cebu (Tenazas 1985) et sur la petite île de Buad au large de la côte ouest de Samar (Cherry 1978; Bellwood \& Koon 1989). Un autre exemple de ce type de production laminaire provient de l'archipel des îles Talaud dans l'abri Leang Tuwo Mane'e sur l'île de Karakellang (Bellwood 1976; 1985; 2007) et à Leang Sarru (Tanudirjo 2005). 

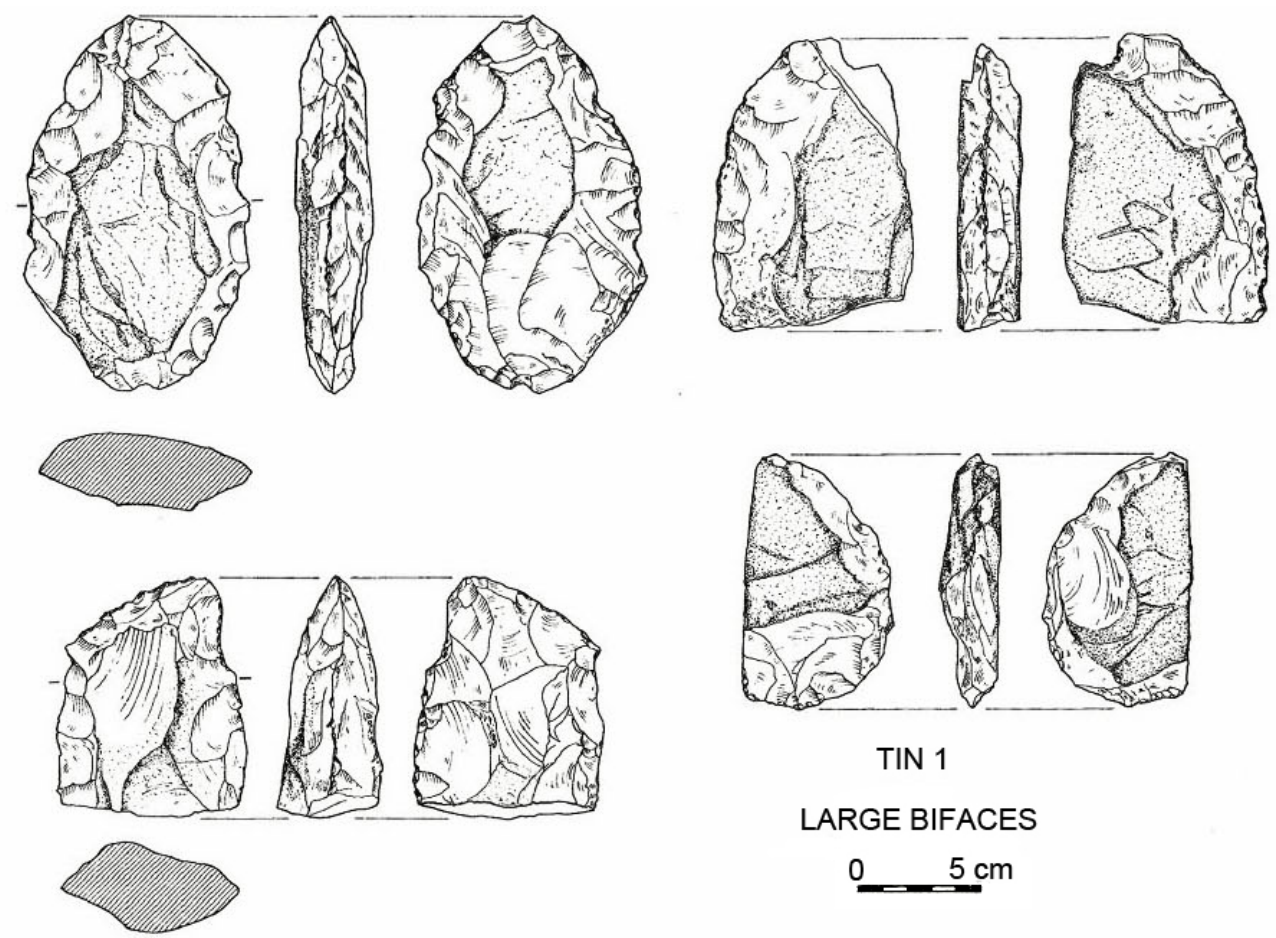

TIN 1

LARGE BIFACES

$0 \quad 5 \mathrm{~cm}$

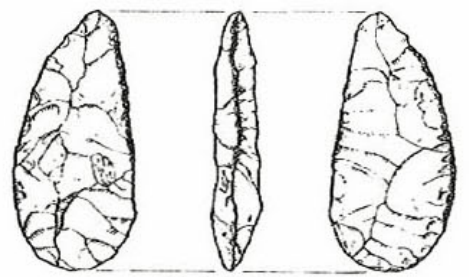
LANCEOLATE
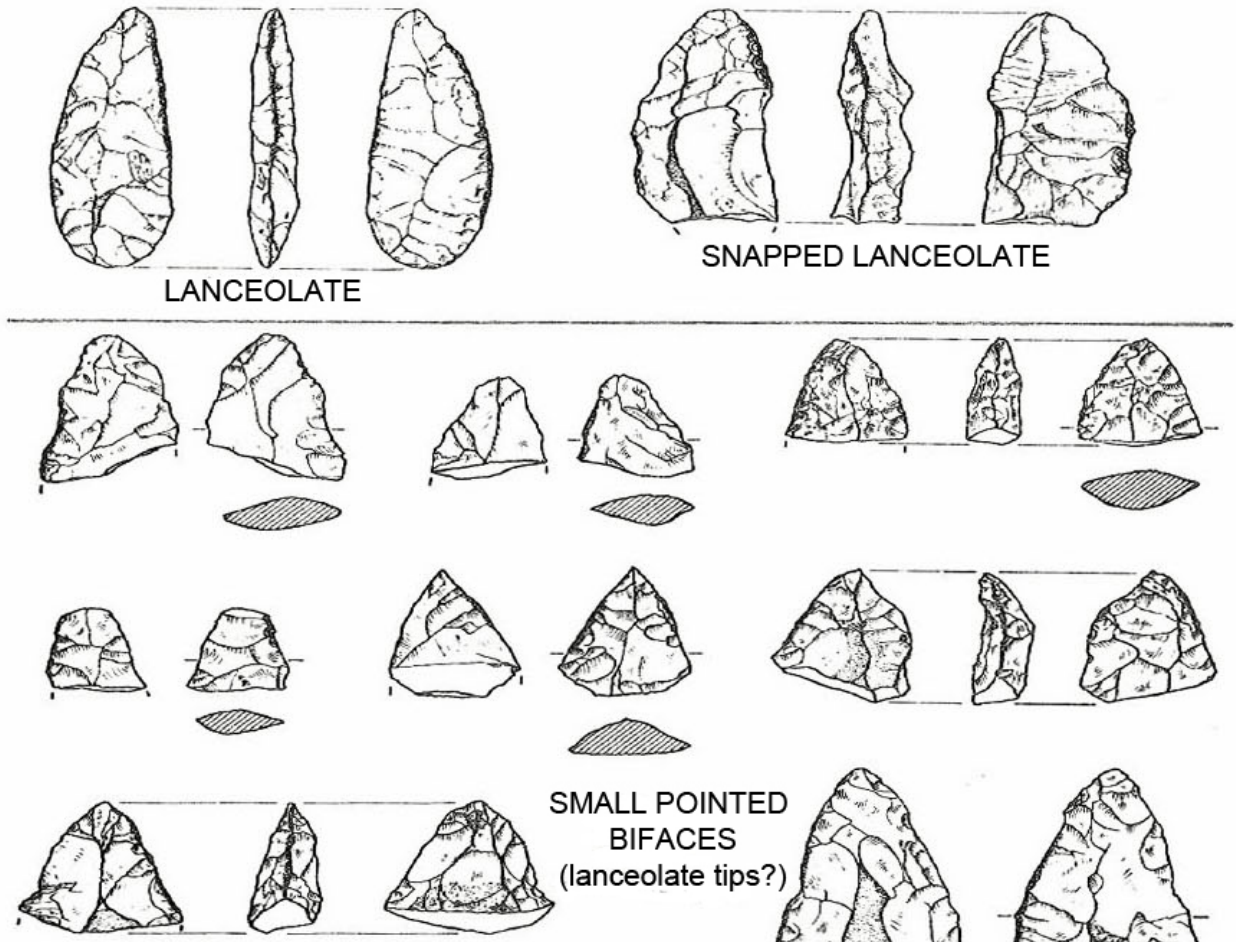

SMALL POINTED BIFACES
(lanceolate tips?)

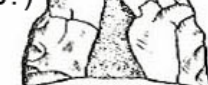

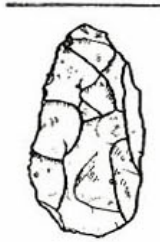

TIN 3
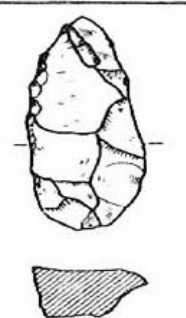
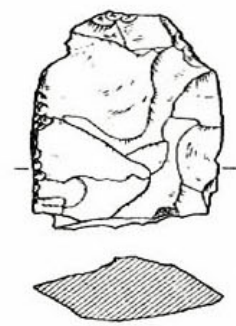

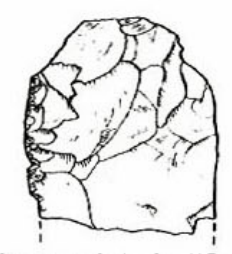

Lanceolate butt?

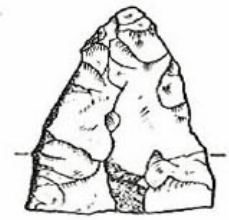

Figure 21. Pièces bifaciales de Tingkayu, Madai-Baturong à Sabah. (d'après Bellwood 1983: 42, 76). Figure 21. Bifacial pieces of Tingkayu, Madai-Baturong à Sabah. (after Bellwood 1983: 42, 76). 

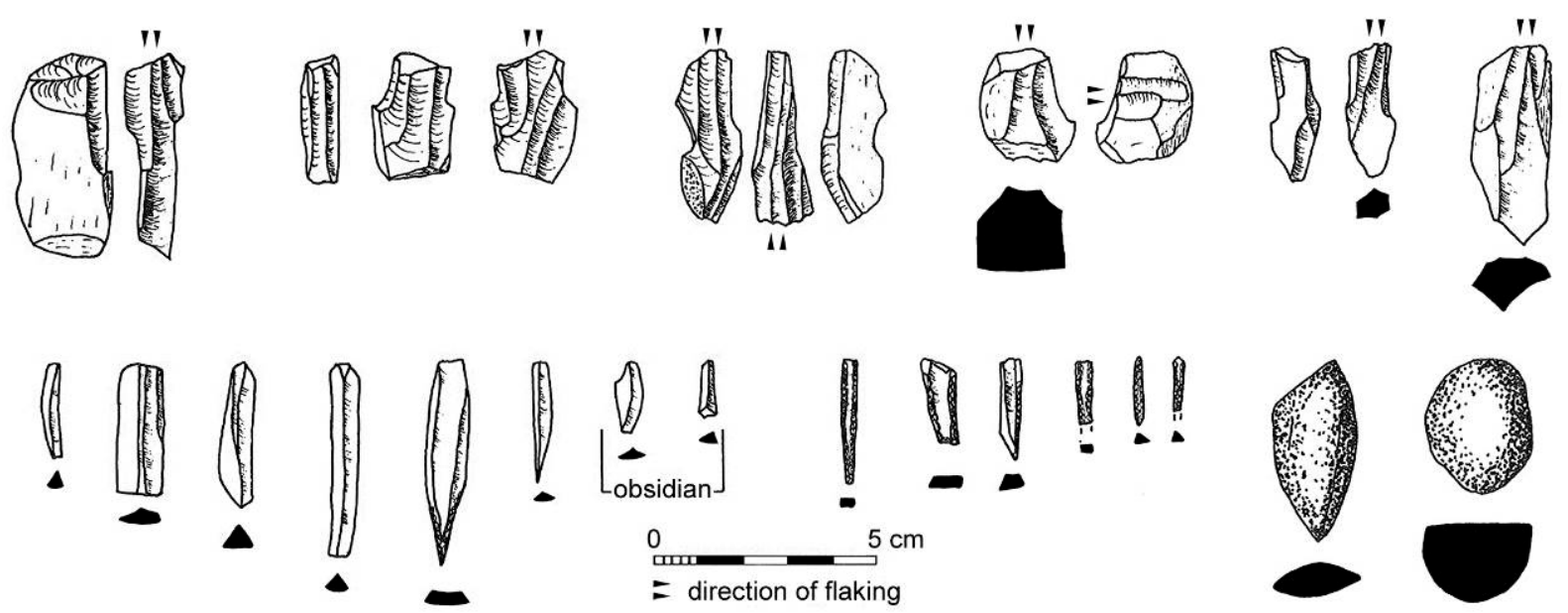

Figure 22. Assemblage lithique de Bukit Tengkorak (Sabah, Bornéo). En haut nucléus à lamelles en agate, en bas, lamelles d'agate et d'obsidienne, micro perçoirs. (d'après Bellwood 1989: 145).

Figure 22. Lithic assemblage of Bukit Tengkorak (Sabah, Bornéo). Top: bladelet core in agate ; bottom: agate and obsidian bladelet, micro-drill. (after Bellwood 1989: 145).

\subsubsection{Kalimantan}

Les données concernant les industries lithiques et les méthodes de taille y sont peu nombreuses. Seules quelques études de sites ont donné des informations permettant de mieux cerner les modalités de productions lithiques à la transition Pléistocène final Holocène (Arifin 2017). Nous retiendrons surtout les sites (Figure 23) de la haute Birang, du Karst de Mangkalihat et, dans une moindre mesure, ceux des monts Meratus, Kalimantan sud (Gua Babi, Gua Payung), de la rivière Kapuas, Kalimantan ouest (Nanga Balang) pour lesquels nous disposons seulement de données partielles ou insuffisantes.

Nanga Balang sur la haute Kapuas a été attribué à une occupation néolithique $(\approx 3000$ 2500 BP) avec des herminettes quadrangulaires, des percuteurs, des enclumes et des meules associés à des vestiges céramiques (Soejono 1991: 8; Arifin 2006). Dans le massif des Meratus, le site de Gua Payung montre un débitage d'éclats (Fajari et Kusmartono 2014).

La grotte de Gua Babi (Figure 23) a été découverte avec d'autres sites dans les années 1990 avec une industrie lithique en chert local, avec quelques artefacts en calcaire, calcédoine et obsidienne, qui est taillée selon un débitage orthogonal (Widianto et al. 1997; Arifin 2006).

Les sites du cours supérieur de la Birang comme les abris de Kimanis, Liang Gobel et la grotte de Lubang Payau fouillés par K. Arifin (Arifin 2004; 2006) montrent de nombreuses similarités techno-typologiques, même si Kimanis présente la séquence d'occupation la plus longue et la plus fournie en matériel. La chronologie des niveaux précéramiques holocènes couvre une période qui s'étend de 11270 à 8840 ans BP. Sur l'ensemble de la séquence, l'industrie lithique est composée d'éclats bruts (plus ou moins corticaux) obtenus par un débitage orthogonal expédient (Figure 24). Il n’y a aucun changement apparent dans ces modalités en dépit de l'arrivée de la céramique, tout comme pour les sites étudiés du karst de Mangkalihat (Grenet et al. 2016), les niveaux supérieurs à céramique (Néolithique) étant datés de 4650 à 1270 ans BP. 

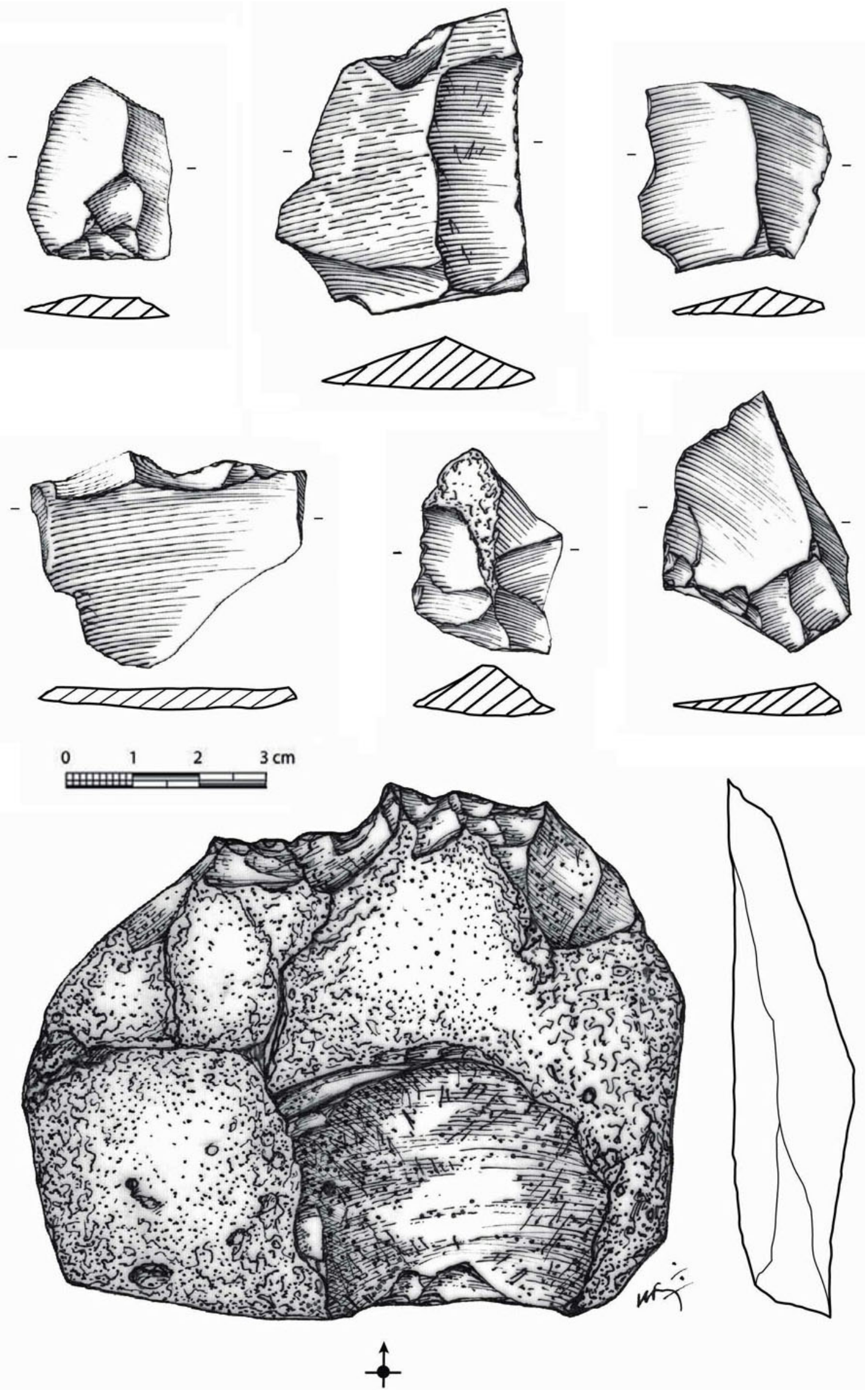

Figure 23. Matériel lithique de Gua Babi, Kalimantan. (Forestier).

Figure 23. Lithic industry of Gua Babi, Kalimantan. (Forestier). 

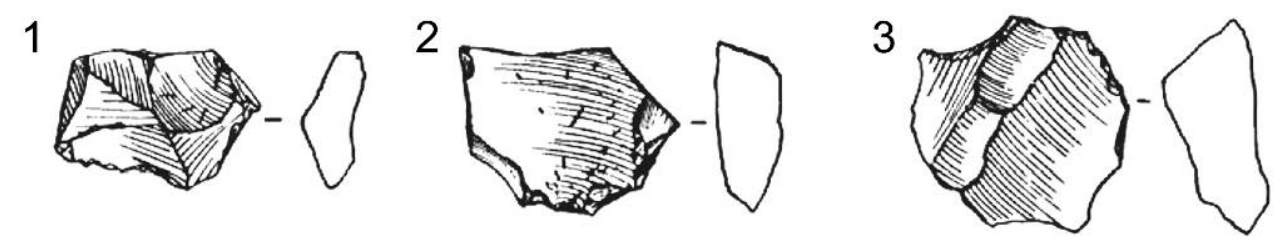

$2 \mathrm{~cm}$
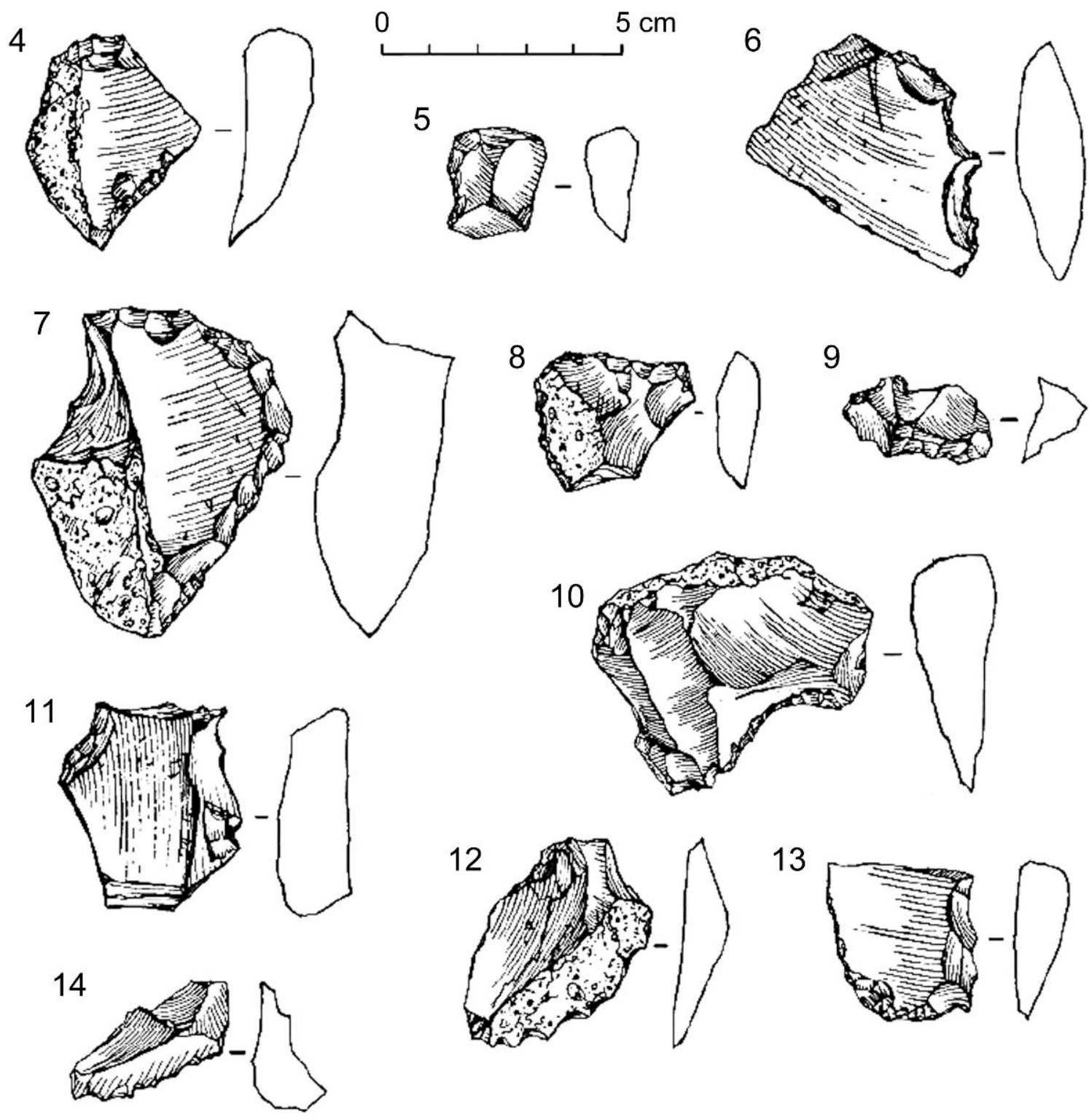

13

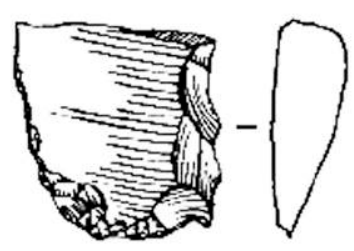

15

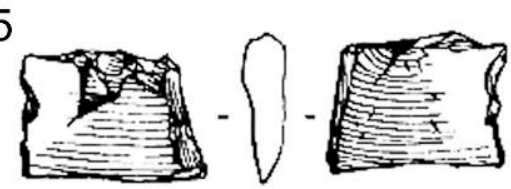

16

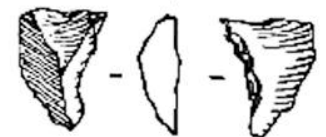

17

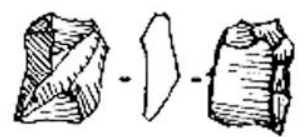

Figure 24. Matériel lithique du site de Kimanis, Kalimantan Est. Test Pit-KMS/TP: 1: éclat brut utilisé; 2: éclat retouché; 3: nucléus-outil. Couche 4 -KMS/C4: 4, 5: éclats utilisés; 6, 7: éclats retouchés; 8 to 17: éclats utilisés et retouchés (d'après Arifin 2004, dessin de H. Forestier).

Figure 24. Lithic material of Kimanis site, East Kalimantan . Test Pit-KMS/TP: 1: used flake; 2: retouched flake; 3: core-tool. Layer 4 -KMS/C4: 4, 5: used flakes ; 6, 7: retouched flakes; 8 to 17: utilized and retouched flakes (after Arifin 2004, drawing by H. Forestier). 
Malgré les très nombreux sites répertoriés dans la région karstique de Mangkalihat (Figure 25), seuls trois ont fait l'objet d'études des vestiges lithiques : Liang Jon (Chazine 2005; Chazine \& Ferrié 2008; Grenet et al. 2016), Liang Abu (Ricaut et al. 2011; Grenet et al. 2016) et Liang Pemalawan (Grenet et al. 2016). Liang Jon est dans la vallée de la rivière Jelai (bassin versant des rivières Marang et Bengalon) en piémont ouest du massif des monts Marang, Liang Abu et Liang Pemalawan, plus au nord, dans la vallée de la Lesan (bassin versant de la Kelai).

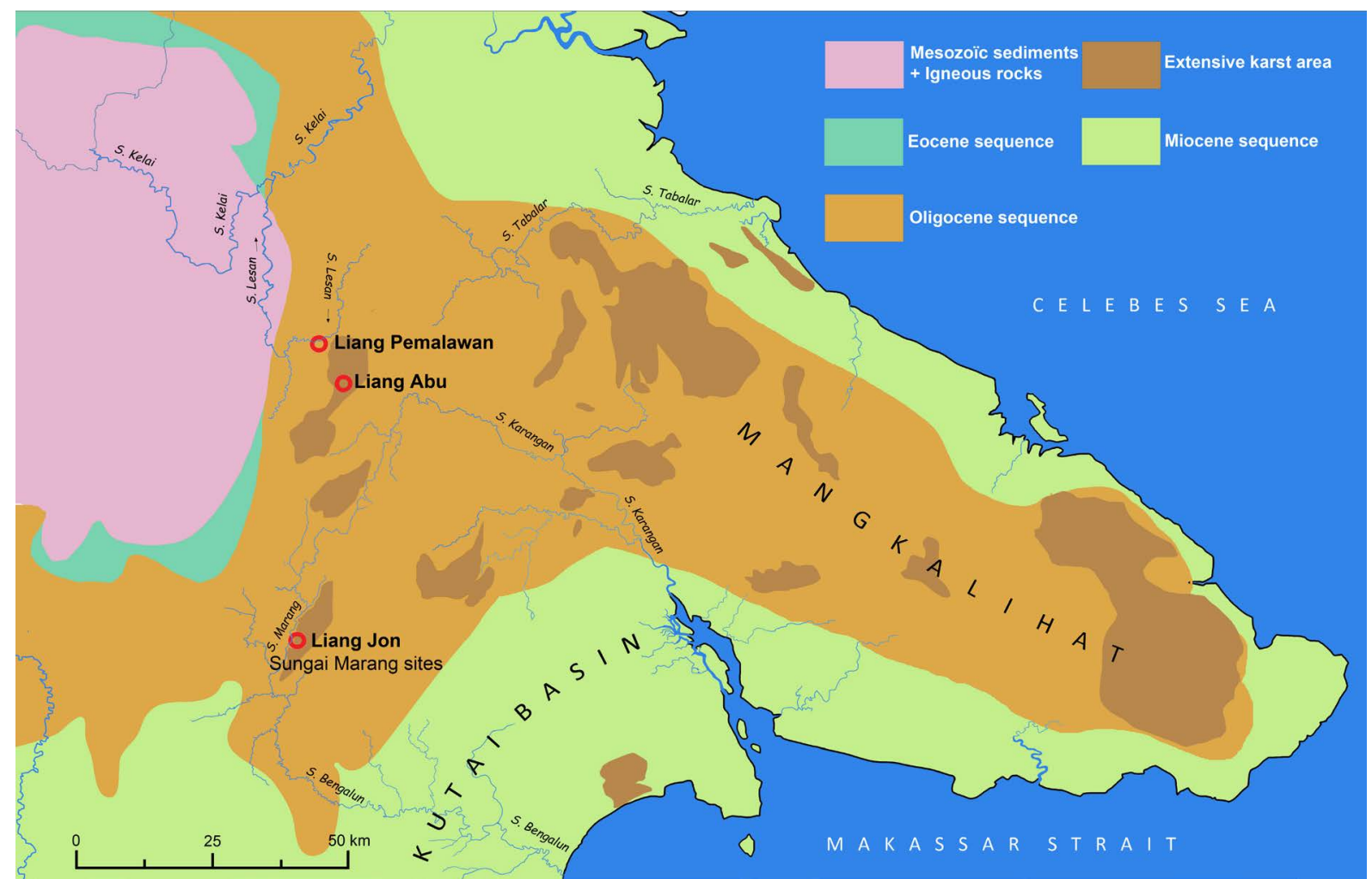

Figure 25. Carte des sites de la région karstique de Mangkalihat, Kalimantan (d’après Grenet et al. 2016). Figure 25. Map of the sites of Mangkalihat karstic area, Kalimantan (after Grenet et al. 2016).

Les assemblages lithiques des sites de la région de Mangkalihat sont très similaires sur un plan morphologique comme technologique. On retrouve le débitage expédient de type orthogonal quel que soit la nature, la qualité et la forme des matières premières utilisées même parfois dans des niveaux récents avec de la céramique comme à Kimanis (Arifin 2004; 2006; Grenet et al. 2016).

La production est orientée vers des éclats de petite taille utilisés bruts ou très peu retouchés. Ce mode opératoire assez simple reste invariant sur l'ensemble des séquences stratigraphiques à Liang Pemalawan (Figure 26) comme à Liang Abu (19761-1672 ans BP) (Figure 27) ou à Liang Jon (10260-995 ans BP, Gay 2010) (Figure 28). Seule la dimension moyenne des artefacts semble évoluer en diminuant du bas en haut de la séquence à Liang Abu et à Liang Jon tout comme à Gua Babi (Widianto 1997; Arifin 2006). 


\section{LIANG PEMALAWAN - US 1}
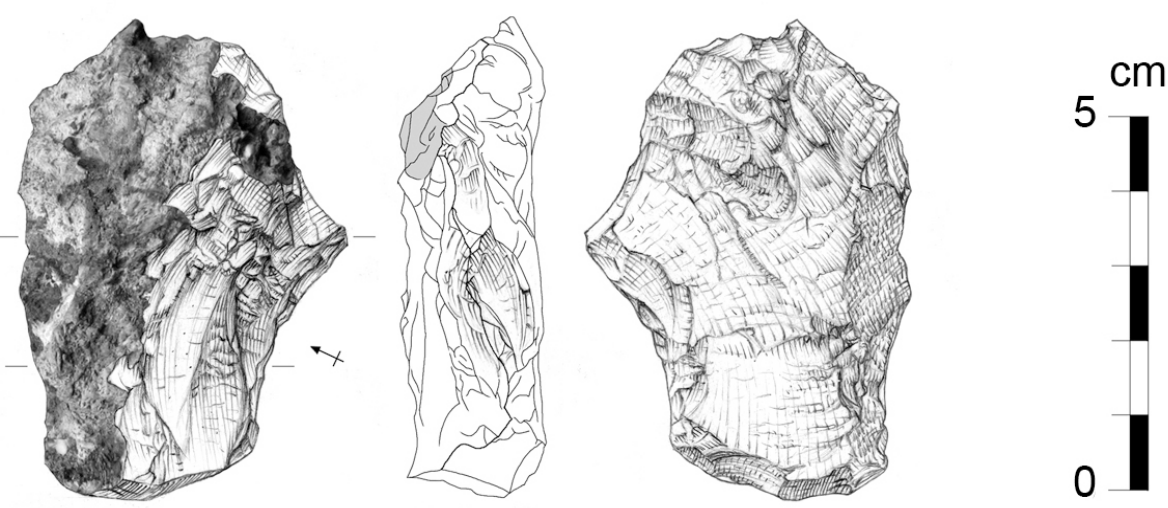

$\mathrm{cm}$

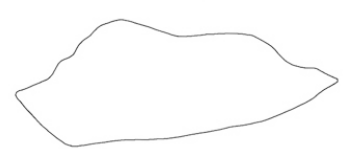

1
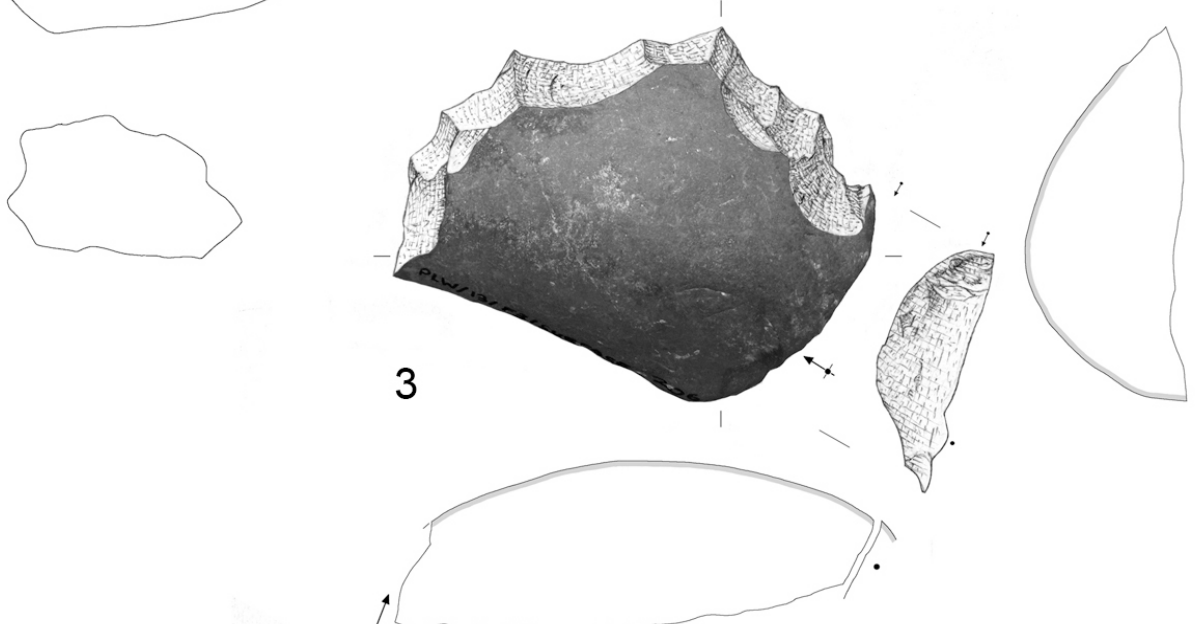

2
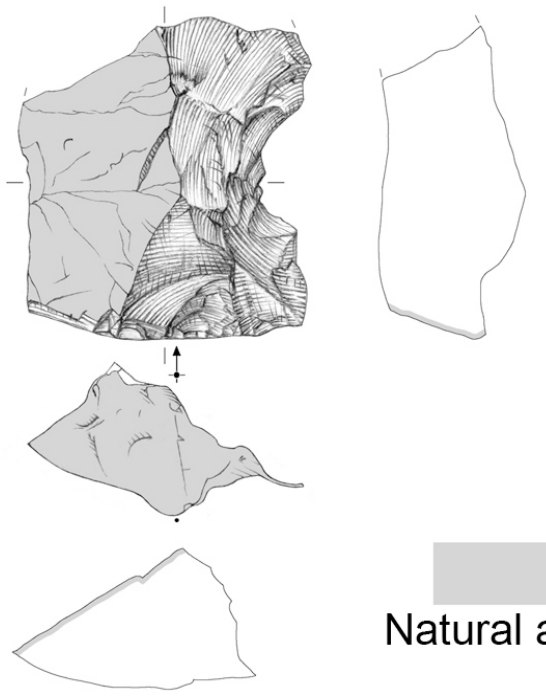

Natural area

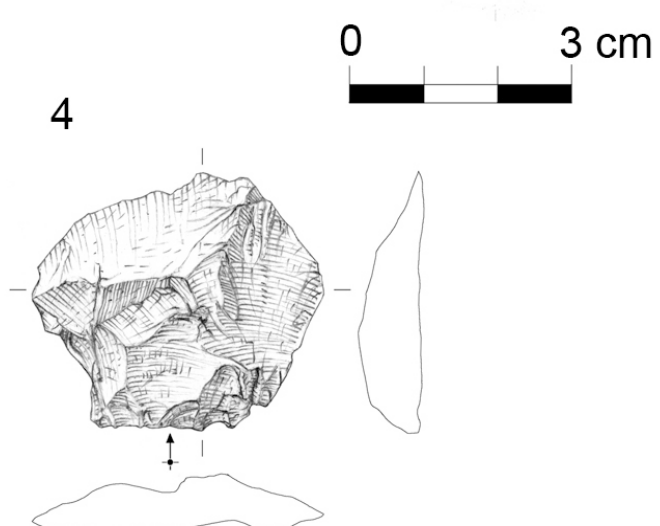

Figure 26. Industrie lithique du site de Liang Pemalawan (Kalimantan Est) : 1: coche latérale sur gros éclat de calcaire; 2: coche latérale sur éclat cortical de galet (chert); 3: denticulé sur éclat cortical de galet (pièce ocrée, andésite); 4: éclat brut (andésite). (d’après Grenet et al. 2016).

Figure 26. Lithic industry of Liang Pemalawan (East Kalimantan): 1: lateral notch on big limestone flake; 2: lateral notch on cortical pebble flake (chert); 3: denticulated on cortical pebble flake (andesite with ochre); 4: flake (andesite). (after Grenet et al. 2016). 


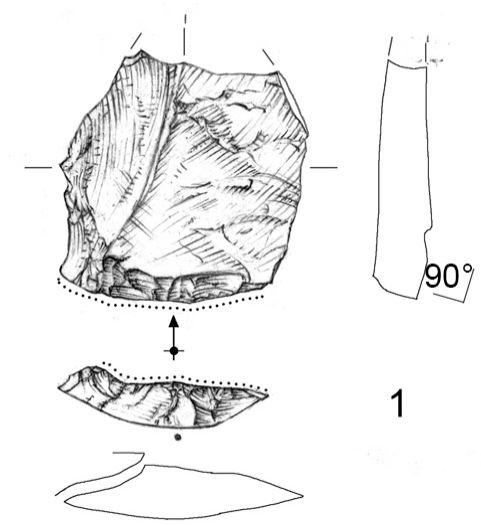

surface naturelle ou corticale
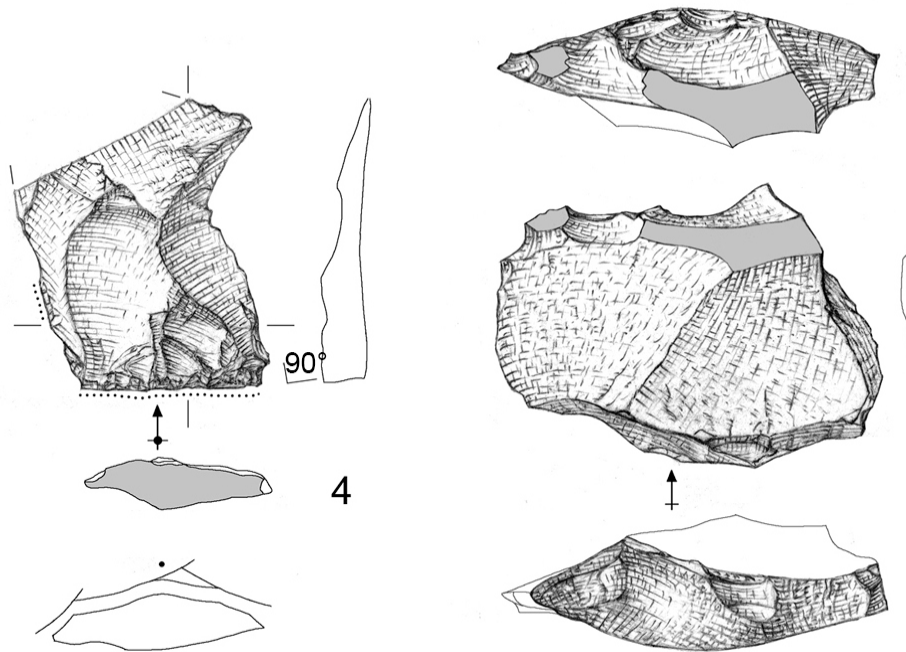
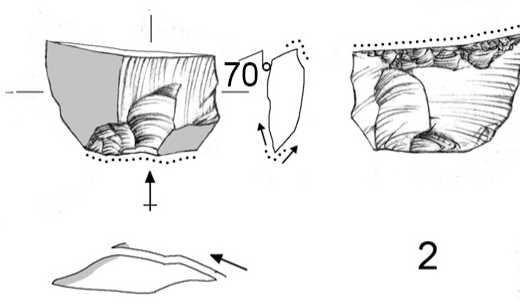

2

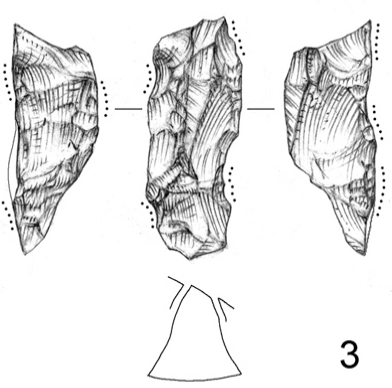

$3 \mathrm{~cm}$

0
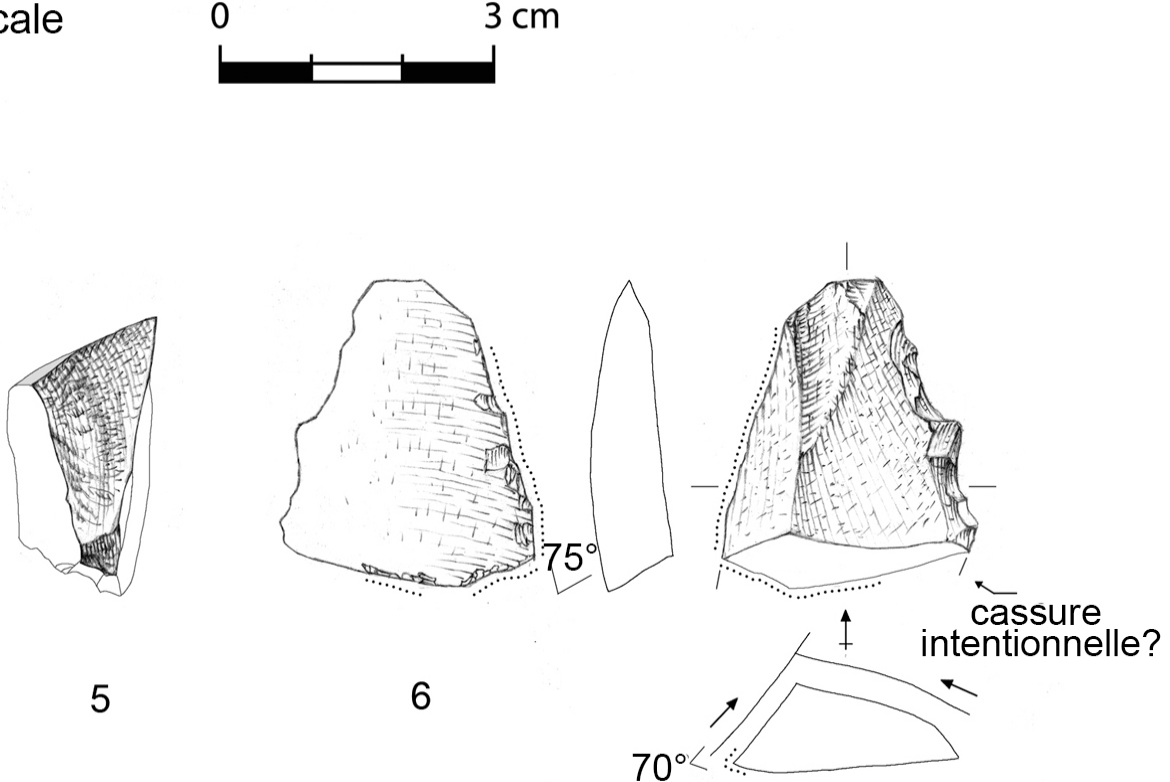

Figure 27. Industrie lithique du site de Liang Abu (Kalimantan Est). 1, 2, 4: éclats bruts utilisés comme outils sur dièdre proximal ou cassure (chert et andésite); 3: nucléus outil (silex); 5: denticulé distal sur éclat cortical (andésite); 6: denticulé latéral sur fragment d'éclat, dièdre proximal sur cassure utilisée (andésite). (d’après Grenet et al. 2016).

Figure 27. Lithic industry of Liang Abu (East Kalimantan). 1, 2, 4: used flake on proximal dihedral or breakage (chert and andesite); 3: core-tool (flint); 5: distal denticulated on cortical flake (andesite); 6: lateral denticulated on flake fragment, proximal dihedral on utilized breakage (andesite). (after Grenet et al. 2016). 


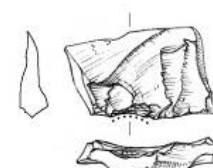

crest
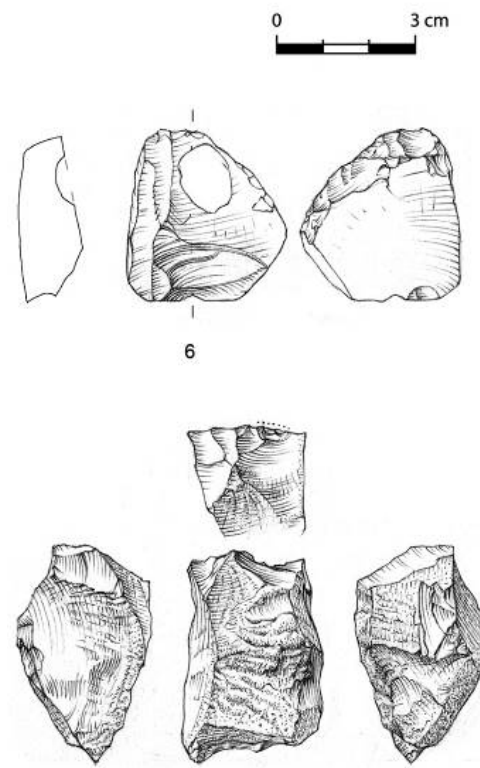

9

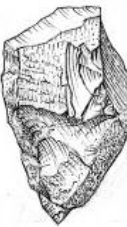

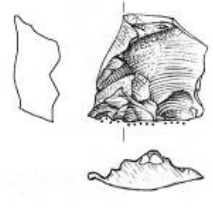
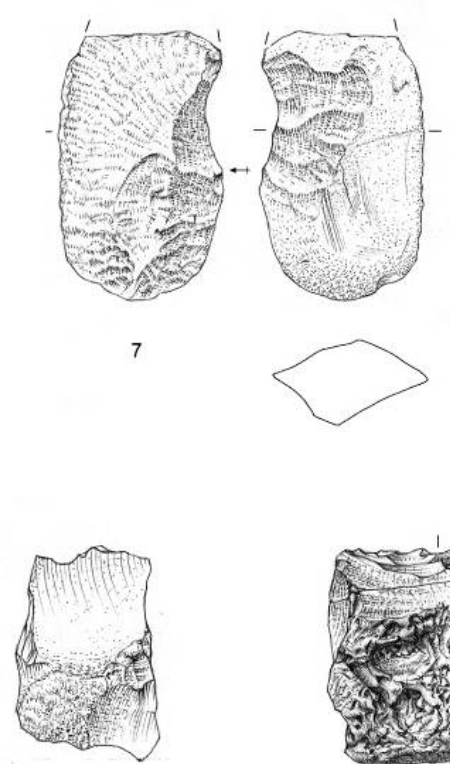

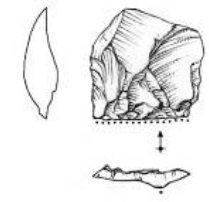

4
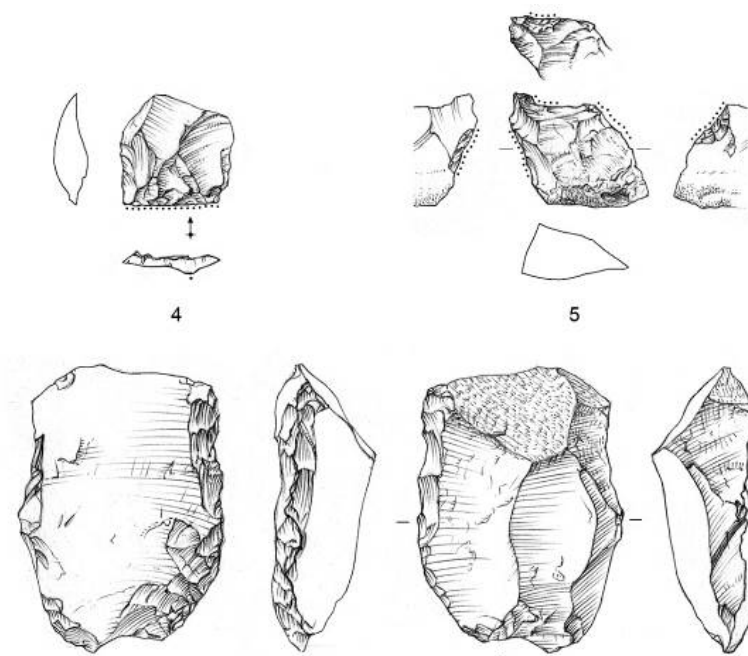

8
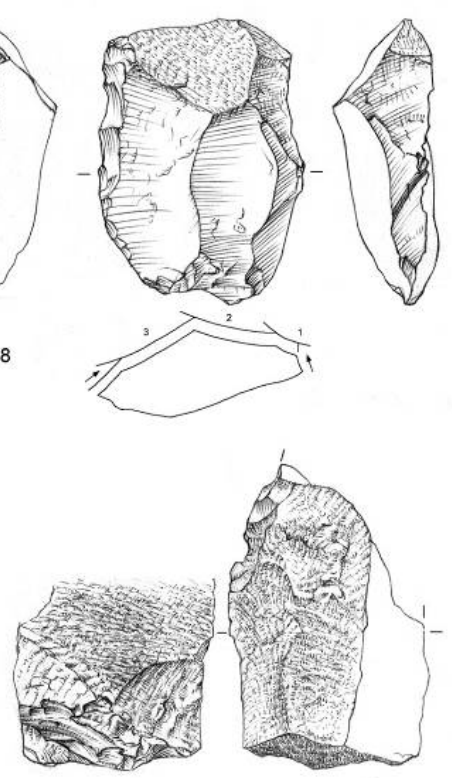

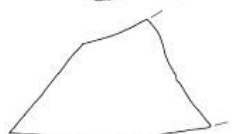

Figure 28. Industrie lithique du site de Liang Jon (Kalimantan Est). 1 à 5: outils sur éclat (silex); 6: Racloir déjeté par retouche inverse (silex); 7: coche latérale sur éclat de galet utilisé par retouche bifaciale (grès quartzite); 8: racloir (silex); 9: nucléus outil (débitage orthogonal, silex); 10: coche distale sur bloc cortical (silex); 11: racloir sur bloc cortical (silex). (d'après Grenet et al. 2016).

Figure 28. Lithic industry of Liang Jon (East Kalimantan). 1 to 5: flake tools (flint); 6: rejected scraper with inverse retouch (flint); 7: lateral notch on pebble flake with bifacial retouch (sandstone-quartzite); 8: scraper (flint); 9: core tool (orthogonal débitage sequence, flint); 10: distal notch on cortical block (flint); 11: scraper on cortical block (flint). (After Grenet et al. 2016). 


\subsection{Les industries lithiques de Sulawesi : le Toalien ou la tentation du laminaire}

Situé au Centre-Nord de l’Archipel, cette île de 175000 km² présente dans son extrémité sud-ouest de nombreux sites datés de la fin du Pléistocène supérieur et du début de l'Holocène (Van Heekeren 1972: 106-125 ; Pasqua \& Bulbeck 1998; Bulbeck et al. 2000). Plus d'une vingtaine de ces sites (Figure 29) ont livré des assemblages attribués au Toalien (Toala signifiant homme de la forêt). Ce sont les sites de Leang Burung 1 (Mulvaney \& Soejono 1970; 1971) et d'Ulu Leang (Glover 1976; 1979b; 1979c) qui ont permis de préciser la variabilité typologique et la chronologie de ce faciès largement représenté par des pointes (armatures).

À Leang Burung 1 l'assemblage lithique est composé majoritairement de chaille (Chapman 1986) et comprend des éléments plus ou moins géométriques à dos abattu qui peuvent être objectivement qualifiés de microlithes, des pointes à dos et des pointes à base concave (plus rarement convexe) et à bords denticulés dites «de Maros » (Mulvaney \& Soejono 1970; 1971; Glover 1976; Pasqua \& Bulbeck 1998; Van Stein Callenfels 1938; Forestier 2007b).

Leur fabrication ne répond pas à une orthodoxie du façonnage telle qu'on pourrait la concevoir dans la confection des pointes de pierre du Paléolithique européen : la symétrie axiale et bilatérale des pointes de Maros est rarement atteinte. En effet, ce type de pointe ne fait en réalité appel qu'à un façonnage partiel et discret des bords, c'est-à-dire sans retouche bifaciale, rasante et couvrante. Les bords sont façonnés sommairement par des petits enlèvements ou par denticulation rentrante bien marquée, avec des retouches directes ou inverses-alternes. Si la forme triangulaire est atteinte ainsi que la base concave, le centre des faces supérieures et inférieures n’est lui pas retouché (Figure 30). Des racloirs sur éclats ou nucléus et des éclats avec des polis d'utilisation (probablement dû au travail de plantes) sont également présents dans le site (Chapman 1981; 1986; Di Lello 2002). L'étude technologique du matériel a mis en évidence le recours à la percussion bipolaire ainsi qu'à une méthode de débitage visant une exploitation laminaire (Chapman 1986; Pasqua \& Bulbeck 1998).

Sur un plan technologique les produits toaliens sont majoritairement des lames. Des lames plus rarement des lamelles qui, pour beaucoup d'entre elles, ont la particularité de n'être pas rectilignes ou standardisées, souvent cassées et mal contrôlées avec des talons et des plans de frappe peu soignés, ce qui atteste d'un mode opératoire assez «maladroit » auquel il manque des étapes de mise en forme du nucléus. Nous n’avons jamais observé de crête ou néo-crête par exemple.

À Ulu Leang (Glover 1976; 1977; 1979b; 1979c), des racloirs à bord abrupt, des pièces à dos microlithiques (croissant, trapèze), des éclats, des petits nucléus (bipolaires) utilisés parfois en outil et des pointes en os ont été découverts dans des niveaux datant de 10000 à 8000 ans BP et marqueraient ainsi le début du faciès Toalien. Il s'agit des premiers signes d'occupation du site. Des lames et des éclats laminaires (allongés) à dos retouché, des pointes de petite taille à base concave (pointe de Maros) et des pointes en os sont présents vers 6000 ans BP. Puis, vers 4000 ans BP ces pointes de Maros se mêlent à des microlithes géométriques et à de la poterie.

Chose rare en Asie du Sud-est, la particularité morphologique des pointes de Maros a fait appel à la création d'un type nouveau qui assure presque à lui seul la définition du faciès Toalien. On retrouve cependant ces pointes associées à une industrie osseuse (notamment des bi-pointes en os dites de Muduk; voir Olsen et Glover 2004), à des racloirs en coquillage ainsi qu'à une faune moderne comprenant notamment Macaca maura, Phalanger ursinus, Sus celebensis, Babyrousa babyrussa (Van Heekeren 1972: 121-122). De plus, le site de Ulu Leang Pattae a non seulement livré un assemblage toalien mais aussi des peintures qui pourraient être associées à ce faciès (Soejono 1984b; Forestier 2007b; 1999) (Figure 29). 

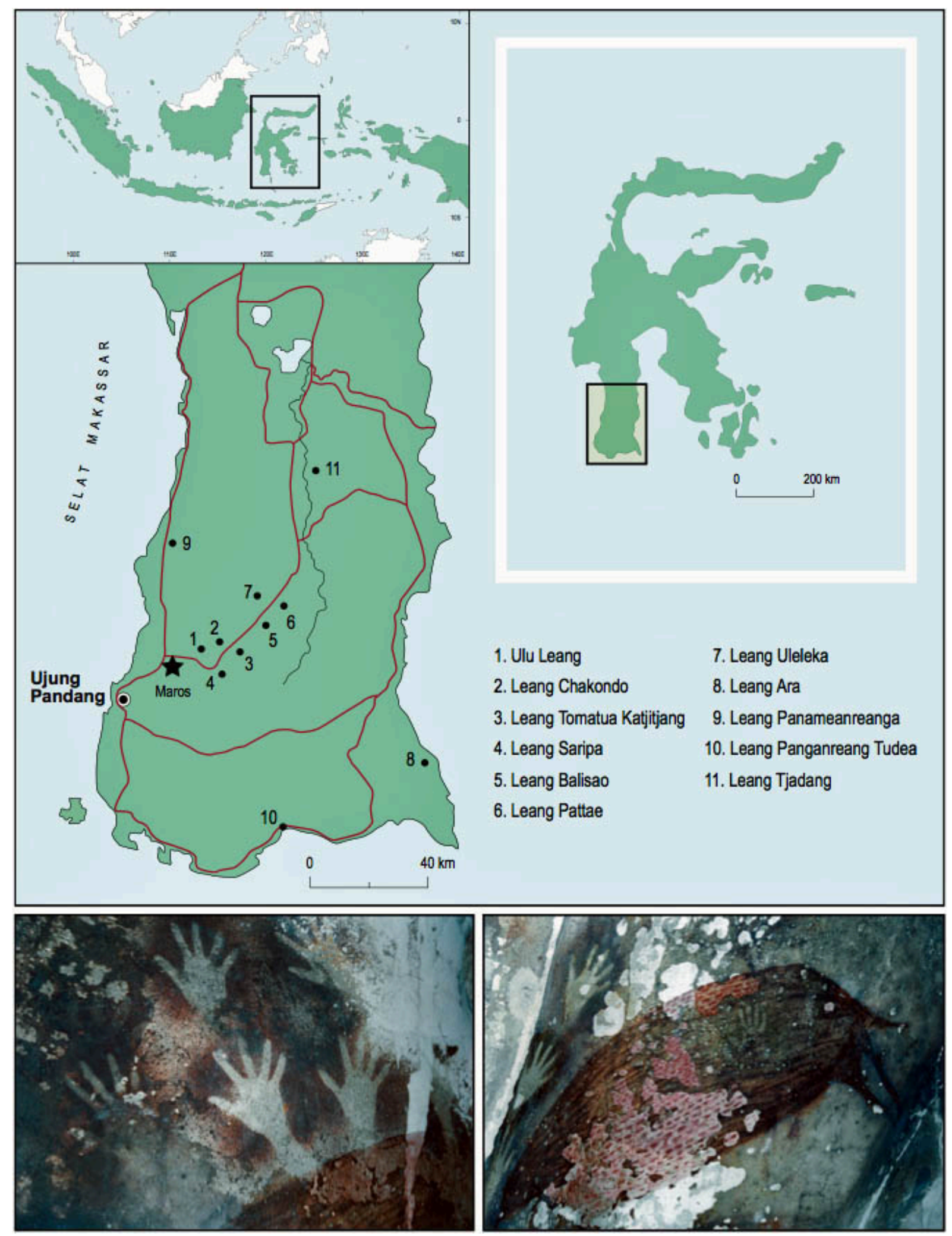

Figure 29. Carte des principaux sites de la vallée de Maros, Sulawesi. (Forestier 2007b).

Figure 29. Map of the major sites of Maros Valley, Sulawesi. (Forestier 2007b). 

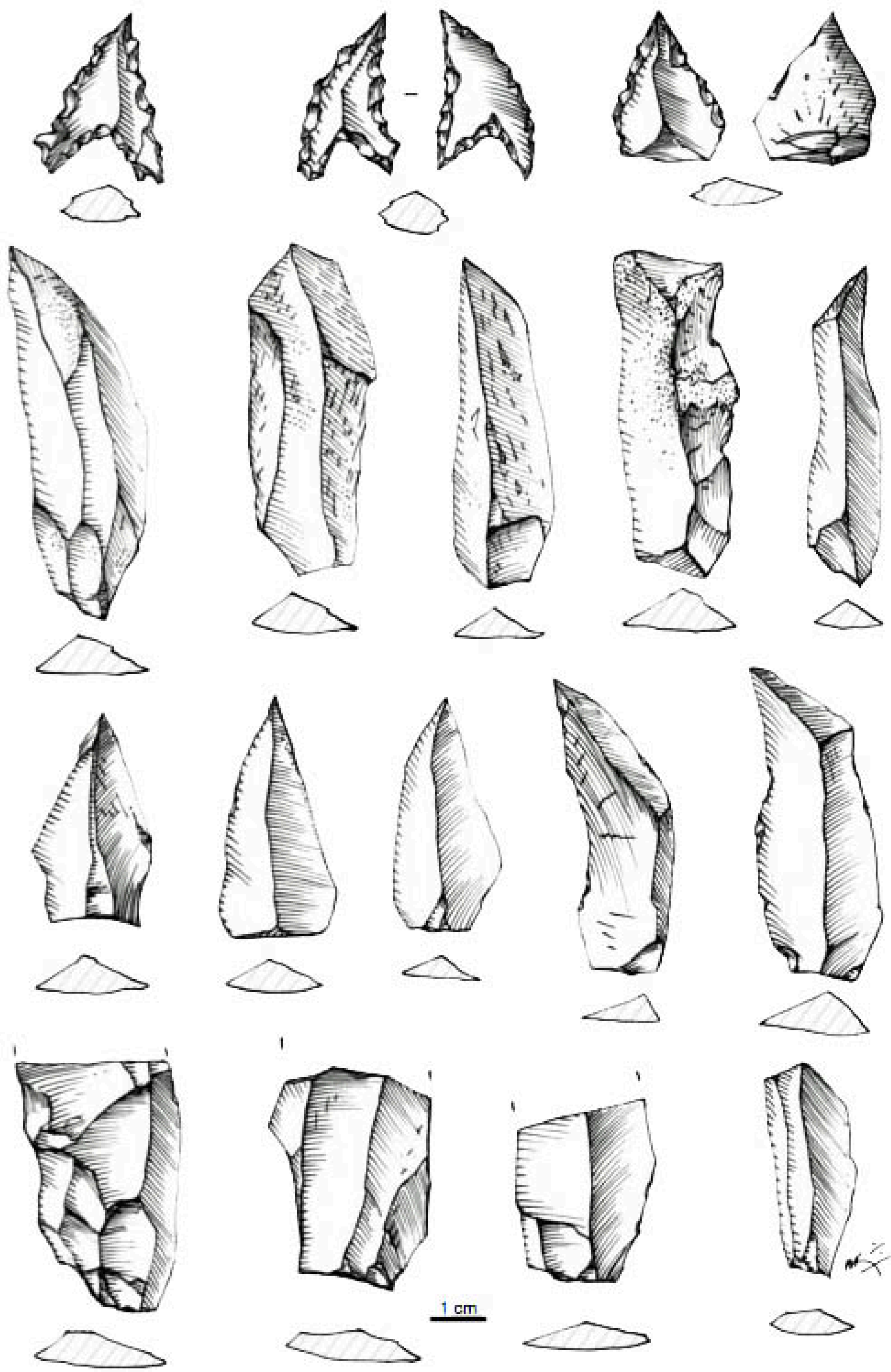

Figure 30. Pointes de Maros et produits laminaires, Maros Valley, Sulawesi. (Forestier 2007b). Figure 30. Maros points and laminar blanks, Maros Valley, Sulawesi. (Forestier 2007b). 
D’autres peintures pariétales ont été découvertes dans la région et ont récemment été datées d'environ 40000 ans BP par séries de l'uranium sur couverts calcitiques (Aubert et al. 2014). Il semble donc que ces peintures soient plus anciennes que le Toalien puisque la chronologie de ce dernier s'étend d'environ 7500 à 1000 ans BP (Bulbeck 2004) avec une présence maximale de pointes de Maros aux alentours de 4000 ans BP mais pouvant varier selon les sites (Pasqua \& Bulbeck 1998; Presland 1980). La présence de microlithes associés peut être antérieure ou postérieure à l'apparition des pointes de Maros. Cela conduit Chapman (1986) à conclure qu'aucune séquence typologique ne peut être appliquée universellement à l'ensemble des sites de la région.

La composition du Toalien reste un phénomène unique où la production laminaire mal contrôlée, réalisée certainement à la percussion directe à la pierre dure est constante (Figure 31), une innovation technique sans précédent qui ne s'est pas répandue dans les autres îles de l'archipel mis à part sur l'île Selayar (à environ 30km de la côte sud de Sulawesi) dans le site de Batang Matasapo (Bulbeck et al. 2000; Hakim 2000). De plus, elle présenterait quelques similarités morphologiques avec certaines industries australiennes (Bellwood 2007; Forestier 2007b; Bulbeck 2004; 2008; Rabett 2012).
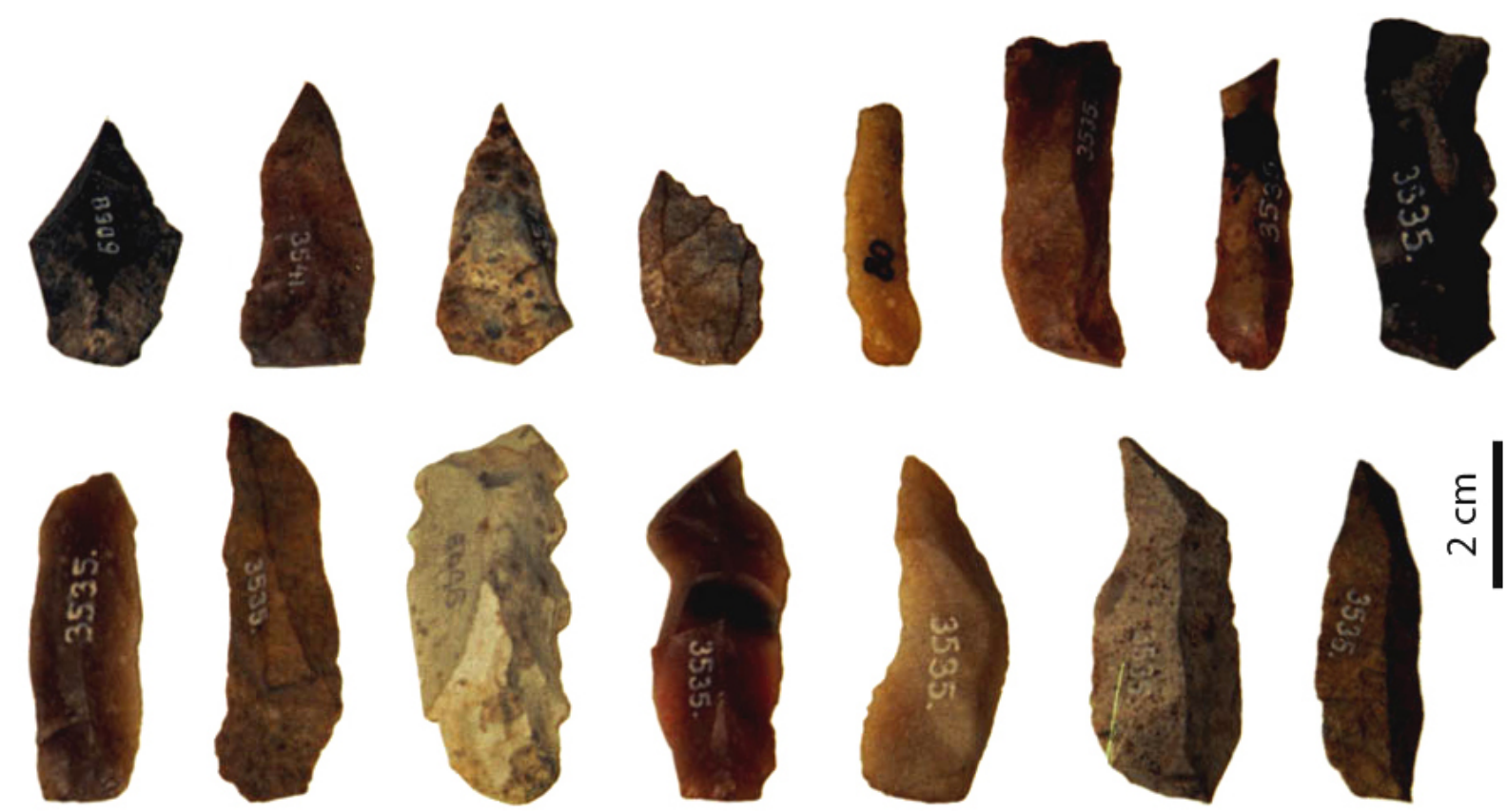

Figure 31. Lames de niveau Toalien, vallée de Maros, Sulawesi (Forestier).

Figure 31. Blades from Toalian level, Maros Valley, Sulawesi (Forestier).

Toutefois, les sites de la région de Maros n’ont pas tous livré des pointes denticulées, des lames et des microlithes qui semblent réservés à des comportements de chasseurs holocènes. C’est le cas de la grotte de Leang Burung 2 (Glover 1977; 1981; Sinha \& Glover 1984; Bulbeck et al. 2004) située non loin de Ulu Leang qui comporte un important remplissage dont les couches les plus anciennes ont été datées par ${ }^{14} \mathrm{C}$ de $31260 \pm 330$ à $23150 \pm 200$ ans BP (Glover 1979b; 1979c; 1981). Aucun changement fondamental au niveau technologique n'est noté tout au long de la séquence si ce n'est une augmentation et une plus grande variété des outils à partir de 20000 ans BP (Sinha \& Glover 1984).

L'assemblage lithique en chaille y est riche et relativement homogène (nucléus, éclats retouchés ou non, débris, etc.). Il est associé à un assemblage osseux important (spatules, pointes, alènes, etc.) et à des coquilles de mollusques. Si certaines pièces ont été considérées par le passé comme Levallois (Glover 1981; Sinha \& Glover 1984), il s’agirait plutôt d'un 
débitage de type Discoïde à forte production de pointes pseudo-Levallois et de supports triangulaires plus ou moins allongés avec parfois un talon facetté (Forestier 2000) (Figure 32).

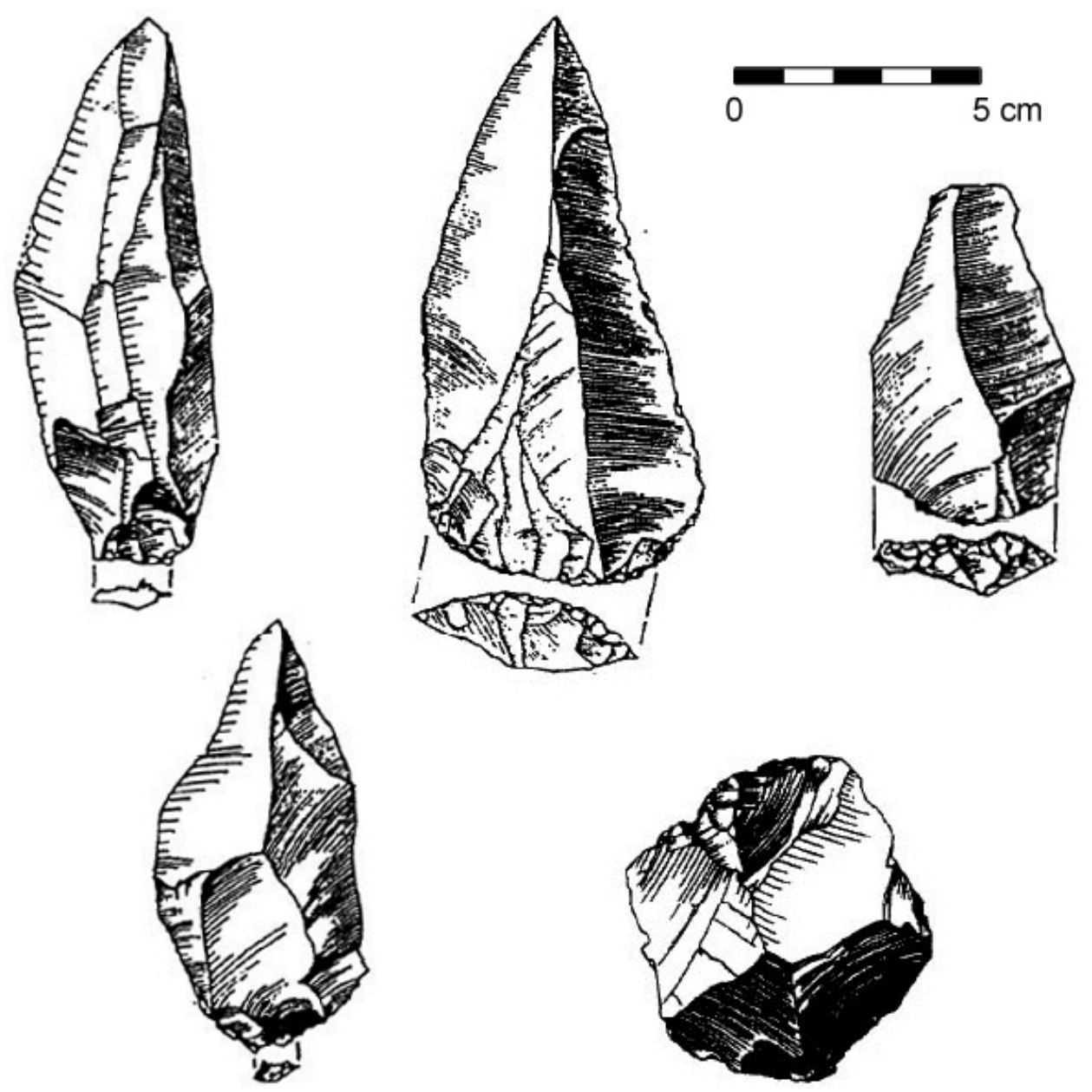

Figure 32. Pointes pseudo-Levallois et nucléus discoïde, Leang Burung2, Sulawesi. (d'après Glover 1977). Figure 32. Pseudo-Levallois points and discoidal core, Leang Burung2, Sulawesi. (after Glover 1977).

Au Nord-ouest de Sulawesi dans l'amas coquillier de Paso, des niveaux datés d'environ 8500 ans BP ont livré un assemblage sur obsidienne dépourvu de pointe de Maros (Bellwood 1976; 2007; Glover 1977; Rabett 2012). Ces pièces (pointes, racloirs et denticulés) étaient associées à des pointes en os. Le choix de supports à base plate avec dos ou des bords abrupts pour la confection des outils a été rapproché des tendances observées dans le pré-Toalien du site d'Ulu Leang 1 (Rabett 2012).

\subsection{Les industries des petites îles de la Sonde : laminaires, spontanées, éphémères et éloignées du carrefour javanais}

Les petites îles de la Sonde de Bali à Timor (Florès, Sumba, Rote, Lembata, Alor, etc.) (Figure 33) sont surprenantes par leur isolement et de fait par l'originalité des industries lithiques rencontrées qui inversent les modes de production jusqu'alors rencontrés i.e. outil sur écla, pointe, galet-hoabinhien (Bellwood 2007; Mahirta 2009). L'éloignement et la discontinuité géographique de ces petites îles (extérieures) ont favorisé l'émergence d'outils et de supports d'outils originaux en réponse à des environnements spécifiques. Avec ces petites îles de la Sonde, une nouvelle idée de la diversité et de l'adaptation à des environnements plus secs moins humides se matérialise dans les outils de pierre taillée et $a$ fortiori des changements économiques. 


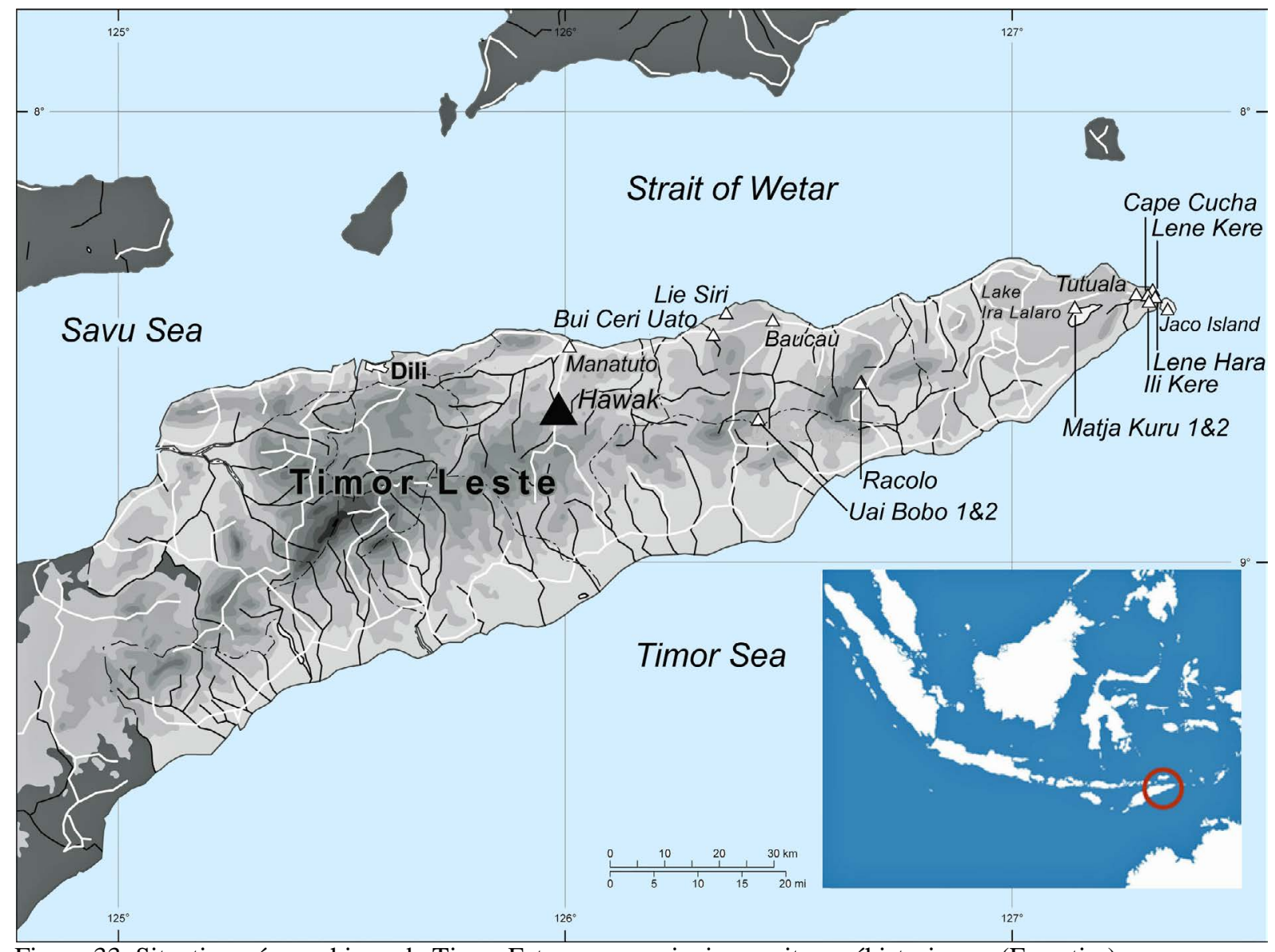

Figure 33. Situation géographique de Timor Est avec ses principaux sites préhistoriques. (Forestier).

Figure 33. Geographical location of East Timor with the major prehistoric sites. (Forestier).

\subsubsection{Les industries lithiques de Timor Est}

Les productions lithiques de Timor passent à ce jour pour être les plus originales car inconnues dans le reste de l'archipel avec notamment les sites de Bui Ceri Uato ou Lie Siri compris entre 9000 et 1000 ans BP et ceux de Uai Bobo 1 et 2 entre 15000 et 1000 ans BP (Glover 1972; 1986; Rabett 2012).

Ces assemblages (Figure 34) s'inscrivent en rupture typologique et technologique avec les industries que l'on a coutume de rencontrer dans l'archipel javanais. Ces productions originales viennent rompre avec une certaine monotonie techno-typologique axée sur une production « ordinaire » d'éclats selon un mode opératoire globalement orthogonal discoide.

Les niveaux I et II du site littoral de Bui Ceri Uato relèvent clairement d'une production d'orientation laminaire ou « elongated, blade-like scrapers » (Glover 1986: 103; Rabett 2012: 168-172), plus exactement d'éclats allongés assez standardisés détachés à la pierre dure. Les nucléus sont à plans de frappe multiples avec une organisation de la production orientée vers un allongement des supports qui peuvent être appelés «lames » par la dimension et la morphologie générale (Glover 1986: 135). Toutefois, une organisation «classique » du débitage à l'identique de celle connue en Eurasie et en Afrique (crête, néo-crête, cintrage, carène, tablette, etc.), est absente de ce système qui produit des supports plutôt larges, épais et souvent mal contrôlés. Le degré d’investissement technique est ici assez faible, ce qui expliquerait le manque de régularité des supports dans leur grande majorité mais aussi le manque de contrôle du débitage (étroitesse, longueur et épaisseur, accidents de taille). 

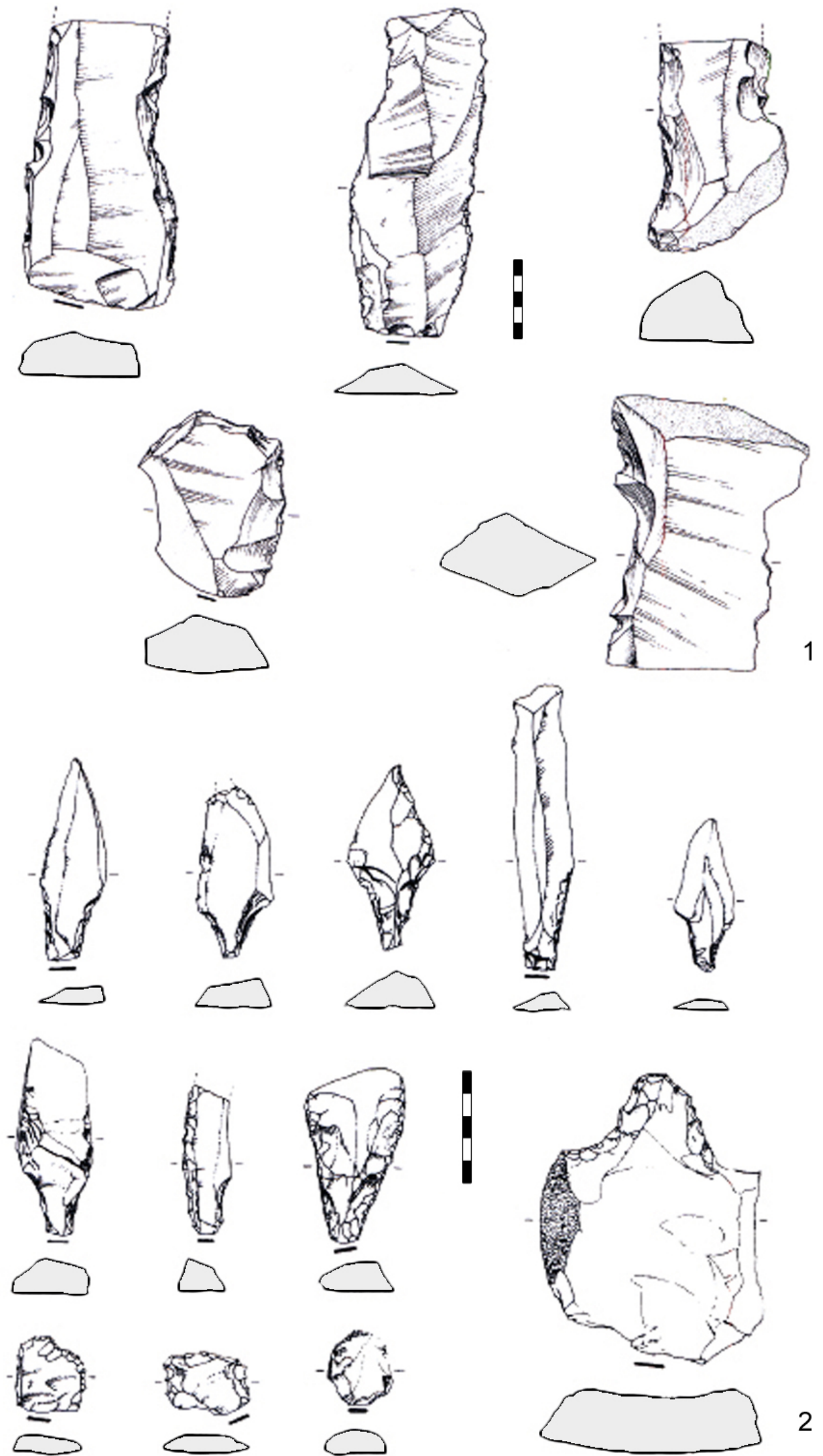

2

Figure 34. Exemple de matériel lithique de Timor Est. (d’après Glover 1986). 1. Lames retouchées, niveau I-II, Bui Ceri Uato. 2.: Pointes pédonculées et lames retouchées, Uai Bobo I, niveau V to IIIc.

Figure 34. Example of lithic material of East Timor. (after Glover 1986). 1. Retouched blades, level I-II, Bui Ceri Uato. 2. Tanged point and retouched blades, Uai Bobo I, level V to IIIc. 
Les outils rencontrés sont étonnants car ce sont des outils typologiquement "paléolithiques supérieurs » majoritairement confectionnés en bout de lame comme des grattoirs simples qui peuvent être onguiformes, des burins ou des racloirs.

Uai Bobo 1 (niveaux de $\mathrm{V}$ à IIIc) est l'autre site de Timor découvert et fouillé par I. Glover (1986) à l'intérieur des terres (environ 200 m d'altitude) qui présente un débitage laminaire et lamellaire avec des nucléus à plan de frappe multiple dont le type d'outil le plus emblématique est la pointe pédonculée.

Ce matériel est une production originale sur support laminaire qui se caractérise par la confection de pointes et d'éléments en chert à pédoncule avec une extrémité distale (apicale, ovalaire ou à tranchant transversal-oblique) à laquelle il faut associer de nombreux grattoirs parfois de très petite dimension, des burins, des outils sur galet et des racloirs ainsi que des lames retouchées (Figure 34). Ce choix technique ne connaît pas de précédent (d'origine) dans l'archipel et se limiterait même à la partie centrale de l'île. Invention spontanée ou phénomène purement de convergence, l'industrie lithique de Uai Bobo I demeure à la fois une spécificité timoraise et une inconnue dans la mosaïque des industries indonésiennes tout en participant à leur diversité sans effet évident de diffusion.

Le seul phénomène géographiquement très éloigné connu à ce jour en Asie qui, dans ces formes les plus initiales, rappellerait ces pointes pédonculées se situe en Corée du Sud dans des niveaux entre 35000-38000 et 20000 ans BP associées à un débitage micro-lamellaire (Nelson 1993; Lee \& Yun 1993; Kim \& Lee 2006; Seong 2008) et dans une moindre mesure au Japon. D'autres cas plus proches et plus récents sont connus dans l'aire de la Papouasie Nouvelle-Guinée avec les "tanged blades" des Highlands (Christensen 1975) ou les spectaculaires types d'outils en obsidienne "stemmed tools » du niveau pré-Lapita daté entre 5000 et 3000 B.P. à Boku Hill sur l'île de Nouvelle Bretagne (Torrence 2004; Torrence et al. 2013).

Plus à l'Est dans l'île, d'autres sites affichent d'autres assemblages lithiques plus conformes à ce que l'on trouve généralement en Indonésie, c'est-à-dire un débitage d'éclats selon une méthode de taille orthogonale (SSDA) à nucléus prismatiques ou polyédriques plus rarement discoïdes. Ces sites en grotte et abri sont les plus anciens de l'île comme par exemple Lene Hara et Jeremalai respectivement datés de 35000 et 42000 ans BP (O’Connor et al. 2002; O’Connor \& Veth 2005; O’Connor 2007). Beaucoup d'entre eux sont souvent associés à un art préhistorique aux motifs régionaux spécifiques de la West Papua Area et daté par U-series entre 29000-24000 et 6000 ans BP (O’Connor 2003; Arifin \& Delanghe 2004; Aubert et al. 2007). Notre propre expérience sur le terrain à Timor lors de la fouille d'un abri Hatu Wakik ou Hawak dans la région de Manatuto (Figure 35) a permis de mettre au jour une séquence archéologique holocène comprise entre 5000 et 3000 ans BP. Celle-ci vient confirmer un débitage orthogonal-prismatique ou discoïdal avec des outils sur éclats de petite dimension largement corticaux comme des coches, des racloirs, des burins et de rares becs (Forestier \& Guillaud 2013). Un art pariétal qui, de toute évidence, est d'époque nonaustronésienne (mains positives et négatives, traits, etc.) y a aussi été découvert par l'un d'entre nous (H. F.). 

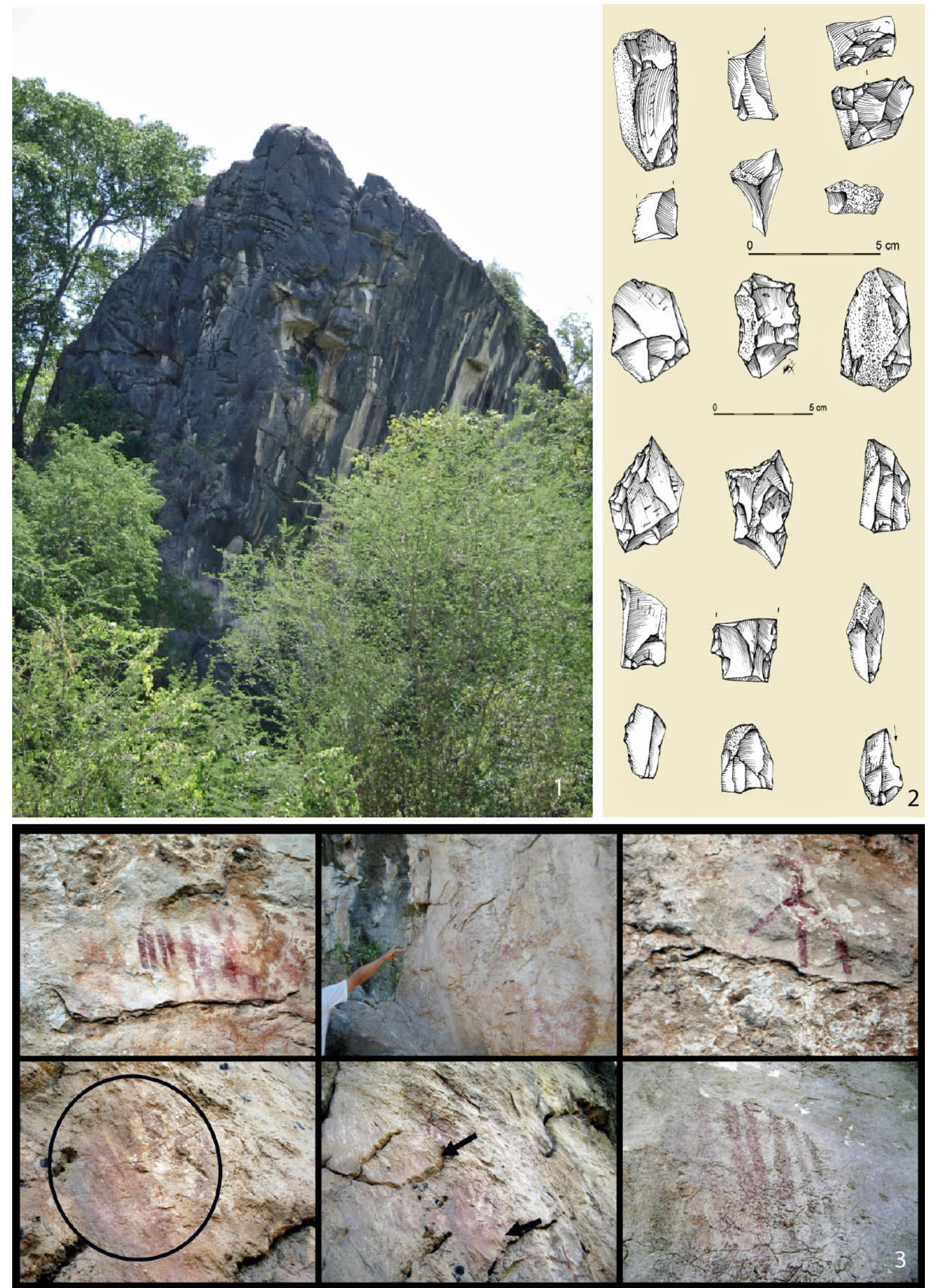

Figure 35. Art préhistorique (3) et matériel lithique (2) du site Hatu Wakik -Hawak (1), région de Manatuto, Timor Est. (H. Forestier).

Figure 35. Prehistoric rock art (3) and lithic material (2) of Hatu Wakik-Hawak site (1), Manatuto region, East Timor. (H. Forestier). 


\subsubsection{Industries lithiques et hommes modernes à Florès : une alliance malaisée.}

Succédant à Homo floresiensis, la présence d'Homo sapiens à Liang Bua est attestée vers 17000 ans BP (Brown et al. 2004; Morwood et al. 2004; 2005; Moore et al. 2009). Des études assez récentes (Moore \& Brumm 2007; Moore et al. 2009) suggèrent la continuité sur le temps long des comportements techniques et d'approvisionnement en matière première. C'est-à-dire depuis les occupations pléistocènes des sites par les différentes espèces d'hommes qui s'y sont succédées jusqu'à l'Homme moderne inclus (Bellwood 1997; Moore et al. 2009; Brumm et al. 2006: 627).

La chaîne opératoire proposée à Liang Bua par Moore et ses collègues (2009) ne se distingue en rien des industries sur éclats du Pléistocène moyen. Elle est sensiblement la même qu'à Mata Menge ( 800000 ans, $c f$. 4.2 supra) à ceci près qu'un type de nucléus bipolaire s'ajoute à ceux déjà mis en œuvre sur gros éclats (flake blank cores) produisant des supports kombewa (contact removal flakes d'après Moore et al. 2009). Il existe aussi deux autres types de nucléus: des nucléus centripètes (radial cores) qui sont, selon nous, des nucléus discoïdes auxquels il faut associer des éclats plus larges que longs, débordants et plus ou moins triangulaires (déjetés) et enfin, des nucléus à débitage orthogonal classiques (multiplatform cores) (Figure 36). Nous interprétons les nucléus bipolaires de Liang Bua comme une exhaustion de nucléus centripètes ou discoïdes.

Les niveaux holocènes de Liang Bua (unité $9^{\mathrm{a}}$, 11000-3000 ans BP) s'orientent eux aussi vers une production d'éclats avec des nucléus relevant de modalités de taille centripètes (discoïde) et orthogonales.

À Liang Bua, il existe cependant des différences sensibles entre les niveaux pléistocènes à homininés non anatomiquement modernes et les niveaux holocènes à Homme moderne en termes non pas d'option technique ou technologique, mais au niveau de choix préférentiels des matières premières donc au niveau économique. Par exemple le chert qui ne représente que $17 \%$ des artefacts dans les niveaux pléistocènes atteint $60 \%$ dans les assemblages holocènes. Le lustré fréquent sur de nombreux bords actifs d'éclats d'époque holocène semble aussi indiquer une fonctionnalité différente ainsi que des besoins et des modes de subsistance nouveaux.

\section{Perspectives autour de faciès lithiques anachroniques : la préhistoire indonésienne entre le proche et le lointain}

Établir un bilan sur la préhistoire indonésienne et ses productions lithiques sur près d'un million d'années n'est pas chose facile d'une part à cause de l'immensité de l'espace et d'autre part du fait que la notion de Paléolithique y est difficilement applicable et notamment celle de « Paléolithique supérieur » ou d' "Épipaléolithique-Mésolithique ». C’est notamment le cas avec les fondements d'une tradition laminaire qui semble imprécise dans ce qui deviendra un archipel et dont on cherche encore l'origine et l'évolution. L'originalité de cette préhistoire tient sans doute à son environnement fragmenté, mais aussi à la complexité des évolutions culturelles qui se télescopent ou s'annulent au rythme d'épiphénomènes industriels. Le débitage laminaire rentre dans ce cas.

Si on délaisse la préhistoire du Pléistocène ancien-moyen associée stricto sensu à Homo erectus dans laquelle paradoxalement on trouve une certaine unité expliquée par un "vraifaux air de déjà vu » au titre d'un " Acheuléen asiatique », celle de l'Homme moderne affiche complexité et diversité dans des environnements inter-tropicaux qui ont vu la destruction des supports réalisés en matières organiques.

Contrairement à l'Asie du Sud-Est continentale où il est encore plus difficile de discerner un Paléolithique ancien, moyen et récent du fait de la continuité d'industries toutes réalisées sur galet (Hoabinhien et autres), l’Indonésie qui devient insulaire à la marge du Pléistocène et 
de l'Holocène propose une hétérogénéité des assemblages lithiques sans précédent. C'est-àdire une diversité dans les modalités de production lithique selon différentes chaînes opératoires de façonnage (galet, pointe de type de Sampung, biface) ou de débitage (discoïde, orthogonal et laminaire).
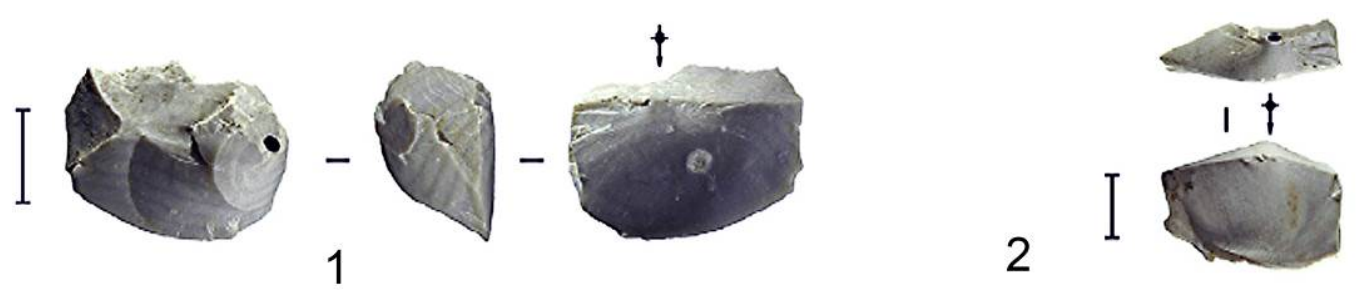

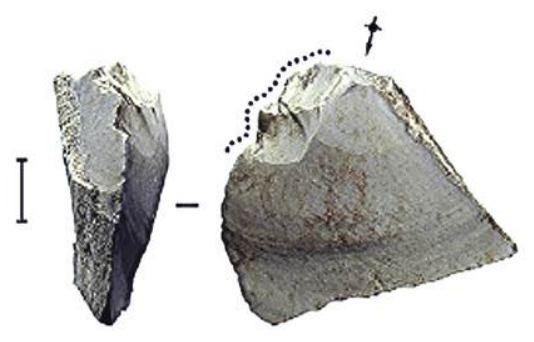

3
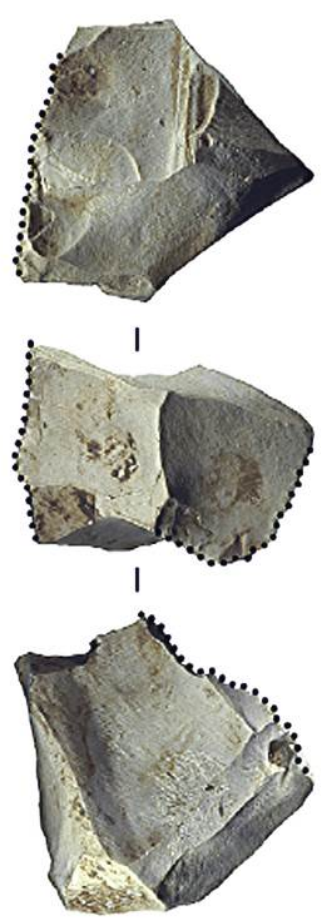

6

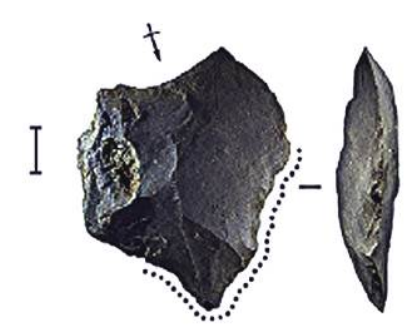

4

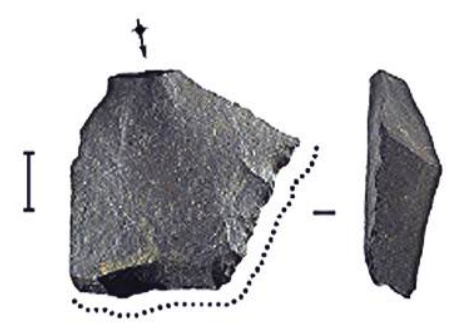

5
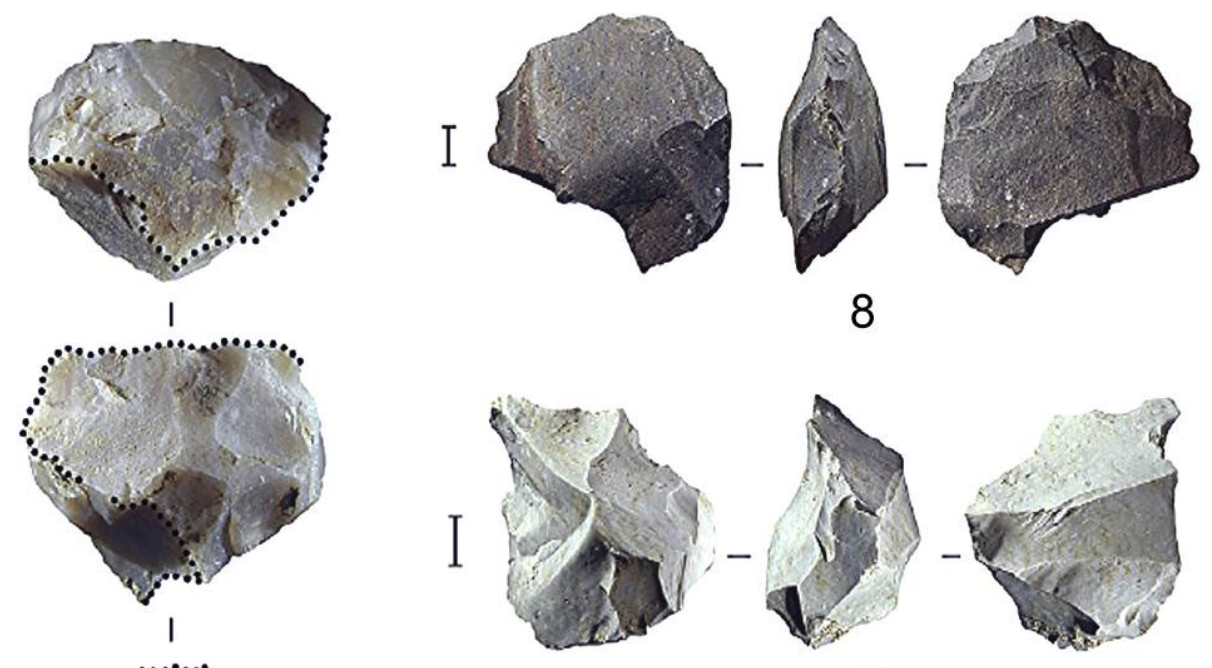

9

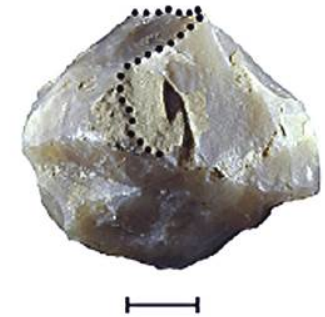

7

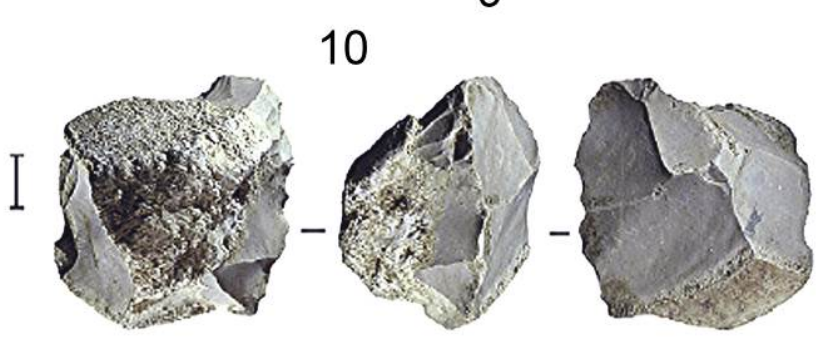

Figure 36. Industrie lithique du site de Liang Bua (Florès), unit 9a. 1, 2: éclats Kombewa; 3, 4, 5: éclats retouchés; 6, 7: nucléus à plans de frappe multiples (débitage orthogonal); 8, 9,10: nucléus discoïdes. (d'après Moore et al. 2009).

Figure 36. Lithic industry of Liang Bua (Flores), unit 9a. 1, 2: Kombewa flakes; 3, 4, 5: retouched flakes; 6, 7: multiplatform core (orthogonal débitage); 8, 9,10: discoidal cores. (after Moore et al. 2009).

Cependant les diverses méthodes de taille rencontrées n’ont pas de valeur chronologique et certains supports lithiques (la lame par exemple) n’ont très certainement pas eu la même 
place au sein de la totalité du système technique (tool-kit) comme c'est le cas en Europe occidentale. Les outils de pierre taillée auraient de fait un sens différent car associés à une autre complexité à jamais disparue, celle du monde végétal et ses dérivés.

Quelle que soit la matière première disponible (silex, jaspe, obsidienne, etc.), le mode de taille le plus représenté est incontestablement le débitage de type orthogonal (SSDA) et dans une moindre mesure, un débitage discoïde (Tableau 1). Si, ces deux chaînes opératoires souvent associées se retrouvent essaimées dans l'archipel, elles respectent néanmoins une distribution centrale dictée par l'axe Sumatra-Java, véritable colonne vertébrale volcanique du Sunda.

D’autres débitages moins diffus comme le débitage laminaire se situeraient loin du « carrefour javanais » dans la marge périphérique de l'archipel indonésien vers les territoires extérieurs comme le Sabah à l'extrême nord de Bornéo, dans l'archipel des îles Talaud, à Sulawesi (Toalien) ou encore à Timor Est qui est la dernière des îles orientales avant le Sahul.

S'il s'agit bien ici d'un bourgeonnement périphérique d'un phénomène laminaire, on ne lui connaît pas d'émergence antérieure comme c'est le cas par exemple, dans le Paléolithique moyen européen du débitage Levallois à production laminaire. L'allongement général des supports s'il est recherché par le débitage laminaire, l'est également parfois dans des modalités orthogonales de production d'éclats (allongés) à la même période dans des îles différentes. Force est de constater que ce que l'on nomme laminaire-lamellaire (laminolamellaire) dans l'Archipel indonésien relève de modalités spécifiques et différentes de celles connues pour l'Europe paléolithique. En effet, le degré de technicité qui se dégage du matériel laminaire indonésien débité à la pierre dure-tendre est plutôt faible, excluant une régularité des produits et une gestion maîtrisée d'une productivité de lames normalisées.

Dans son ensemble, la production n’y est généralement pas soignée telle que le démontre une mise en forme peu orthodoxe du bloc, c'est-à-dire sans aménagement systématique d'une crête frontale et avec un contrôle aléatoire du carénage comme du cintrage du nucléus (flanc et cintre non réguliers). D’un point de vue économique, dès que le plein débitage est atteint il ne porte pas la marque d'étapes classiques de réaménagement par des tablettes même partielles (sommitale, transversale, axiale, etc.). Avec si peu d'investissement technique et pour ainsi dire pas d'évolution locale ou de diffusion, le débitage laminaire se présente en Indonésie comme un fait technique dans sa spontanéité productionnelle la plus brute.

Nous avons l'impression de suivre des faits techniques spontanés, impromptus, émergents sans héritage plus ancien, sans avant ni après qui n'évolueraient pas dans la diachronie comme on voudrait bien l'entendre, c'est-à-dire du simple vers le complexe, du lourd vers le léger (leptolithisation). Mais encore, il s'agirait d'une généralisation d'armatures spécialisées sur lame ou lamelle avec des pièces à dos, des pointes rectilignes ou foliacées, trop rares encore dans les assemblages des derniers chasseurs insulaires.

Une autre idée de la complexification vers l'allégement de l'outillage serait à repenser et qui n'est certainement pas étrangère à la civilisation disparue du végétal induisant une autre portée de l'investissement technique tournée vers les matières organiques (pointe, arme d'hast, liant, colle, fixation, piège, emmanchement, etc.). Il reste à analyser les causes et l'origine du débitage laminaire, mais aussi celles du façonnage de pointes bifaciales et unifaciales associées ou pas à des équipements à tendance microlithique ou géométrique.

On a peut-être jusqu'ici trop eu tendance à simplifier la question en ne cherchant que vers l'Ouest et les chronologies anciennes du peuplement asiatique largement dominées par des outils sur galet que l'on retrouve jusqu'à l'île de Sumatra. Il serait intéressant de reconsidérer le phénomène laminaire indonésien non pas comme un emprunt continental (venu de l'Ouest) mais plutôt comme un phénomène central de transition Asie-Pacifique. Autrement dit, un phénomène d'émergence-convergence qui ouvrirait ensuite à une série de mutations techniques concomitantes à la colonisation du Sahul. 
Tableau 1. Tableau synthétique des chaînes opératoires lithique en Indonésie. (Forestier \& Grenet).

Table 1. Synthetic table of the lithic chaînes opératoires in Indonesia. (Forestier \& Grenet).

\begin{tabular}{|c|c|c|c|c|c|c|c|c|}
\hline & & \multicolumn{7}{|c|}{ Chaînes opératoires } \\
\hline & & \multicolumn{4}{|c|}{ Débitage } & \multicolumn{3}{|c|}{ Façonnage } \\
\hline & & $\begin{array}{l}\text { Chopper ou } \\
\text { chopping tool }\end{array}$ & $\begin{array}{c}\text { Discoïde ou } \\
\text { Pseudo Levallois }\end{array}$ & $\begin{array}{c}\text { SSDA ou } \\
\text { Orthogonal }\end{array}$ & Laminaire & Bifacial & Unifacial & Faciès à pointe \\
\hline \multirow{6}{*}{$\begin{array}{l}\text { Archipel } \\
\text { indonésien }\end{array}$} & Sumatra & PA-HEA & & & & PM-HEA & PS-HAM & \\
\hline & Java & PA-HEA & PA, PM - HEA & PM, PS HEA, HAM & & PM-HEA & & PS-HAM \\
\hline & Bornéo & & & PSF, $\mathrm{H}$ & $\mathrm{H}-\mathrm{HAM}$ & PSF, H-HAM & & \\
\hline & Flores & & $\begin{array}{c}\text { PA, PM, PSF, } \\
\text { H, HEA, HF, HAM }\end{array}$ & $\begin{array}{c}\text { PA, PM, PSF, } \\
\text { H, HEA, HF, HAM }\end{array}$ & & & & \\
\hline & Timor & & & & $\mathrm{H}-\mathrm{HAM}$ & & & PS-HAM \\
\hline & Talaud & & & & $\mathrm{H}-\mathrm{HAM}$ & & & \\
\hline
\end{tabular}

Périodisation

$\mathrm{H}$ : Holocène

PSF : Pléistocène supérieur final

PS : Pléistocène supérieur

$\mathrm{PM}$ : Pléistocène moyen

PA : Pléistocène ancien
Anthropologie

$H A M$ : Homme anatomiquement moderne

$H F$ : Homo floresiensis

HEA : Homo erectus asiatique 
La préhistoire de Sunda et celle du Sahul restent étroitement liées aux fondements techniques de l'Homme moderne, à son émergence et à sa conquête (Balme \& O'Connor 2014). Relevant de causalités environnementales, démographiques et technologiques, l'odyssée australo-tasmanienne présente dans sa préhistoire holocène des ressemblances typotechnologiques avec les industries laminaires à pointes indonésiennes notamment entre certaines pointes à dos abattu comme celles rencontrées dans le Toalien (Sulawesi) avec les armatures australiennes dites de Bondi (Mulvaney \& Soejono 1971).

Il pourrait s’agir du résultat d’une évolution technique commencée au Pléistocène supérieur final dans le semis des îles indonésiennes dont l'aboutissement trouverait unité et recentrement (un autre cul-de-sac) en Australie au début de l'Holocène dans ce que l'on appelle la "Australian small tool tradition »: les pointes denticulées à retouche bifaciale dites de Kimberley, les pointes de Bondi (à dos abattu) et celles de Pirri, les segments géométriques (elouera), les longs couteaux de type Leilira (longue lame), etc. (Mulvaney 1969; McCarty 1976; Mulvaney \& Kamminga 1999). Un autre point analogue est sans doute le «minimalisme » de la chaîne opératoire de production de supports lamellaires et lamellaires australienne (Flenniken \& White 1985).

Ainsi, d'un point de vue technologique, ce que l'on nomme «Paléolithique supérieur » en Indonésie se définit comme un équipement lithique hétérogène, efficace, varié dont les nuances répondent à des choix différents de techniques et d'outils en fonction des environnements et au gré des migrations nourries au régime des échanges et des abandons, des attentes et des ruptures.

La diversité des assemblages et la complexité grandissante des évolutions culturelles en Indonésie doivent interroger sur les degrés d'invention, d'innovation et d'adaptation technique à des environnements changeants ainsi que proposer des modèles théoriques pour expliquer d'autres cas, d'autres isolats, d'autres contextes en marge de la préhistoire européenne.

L’Indonésie préhistorique présente un grand intérêt car elle est influencée par plusieurs facteurs autres qu'humains ou le voyage, notamment ceux de l'endémisme, de la résilience et de l'isolement insulaire (ou pas) qui permettront, peut-être un jour, de donner du sens à des phénomènes comme les discontinuités technologiques sur le temps long, les industries émergentes des confins et des bordures ainsi que l’éclosion de convergences.

\section{Remerciements}

This article is a synthesis about "The lithic productions of Indonesian Archipelago" which was made possible through a long period of scientific cooperation between Pusat Penelitian Arkeologi \& Centre for Prehistoric and Austronesian Studies, Jakarta, Indonesia and French Institutions (MNHN-CNRS). An important part of these field works in Indonesia was funded by the Ministère des Affaires Etrangères et du Développement International (MAEDI), so we would like to thank the Commission des Fouilles Archéologiques à l'Etranger and the SCAC de l'Ambassade de France en Indonésie.

\section{Références}

Allchin, B. 1966, The stone-tipped arrow: Late stone age hunters of the tropical world. London, Phoenix House, London, 224 p. (en anglais) ("La flèche à pointe de pierre: Les chasseurs de l'âge de la pierre du monde tropical”) 
Allen, H. 1991, A review of the late Pleistocene/early Recent stone tool assemblages of Java. Bulletin of the Indo-Pacific Prehistory Association 11: 36-47 (en anglais) ("Une revue des assemblages lithiques du plistocène final/recent de Java.”) doi:10.7152/bippa.v11i0.11371

Amano, N., Moigne, A. M., Ingicco, T., Sémah, F., Rokus Due Awe, \& Simanjuntak, H. T. 2016, Subsistence strategies and environment in late Pleistocene-Early Holocene Eastern Java: Evidence from Braholo cave. Quaternary International, 416: 46-63. (en anglais) ("Stratégies de subsistance et environnement à la fin du Pléistocène et le début de l’Holocène dans l’Est de Java: données de la grotte de Braholo”) doi:10.1016/j.quaint.2015.09.072

Arifin, K. 2004, Early Human occupation of the East Kalimantan Rainforest (the Upper Birang River Region, Berau). PhD Thesis. Australian National University, Canberra, 473 p. (en anglais) (“Occupation Humaine Précoce de la Forêt Tropicale de l’Est de Kalimantan (Région de la Haute Rivière Birang, Berau)”)

Arifin, K. 2006, The Austronesian in Borneo. In: Austronesian diaspora and the ethnogenesis of people in Indonesian Archipelago. (Simanjuntak, H.-T., Pojoh, I. \& Hisyam, M., Eds.), Pr. R. P. Soejono Festschriift, Lipi, Jakarta: p. 146-162. (en anglais) (“L'Austronésien à Bornéo”)

Arifin, K. 2017, Terminal Pleistocene and Early Holocene Human occupation in the Rainforests of East Kalimantan. In: New Perspectives in Southeast Asian and Pacific Prehistory. (Piper, P. J., Matsumura, H. \& Bulbeck, D., Eds.), ANU Press, The Australian National University, Canberra, Australia: p. 97-124. (en anglais) (“Occupation humaine au Pléistocène terminal et au début de l'Holocène dans les forêts humides du Kalimantan oriental”)

Arifin, K., \& Delanghe, P. 2004, Rock art in West Papua. UNESCO, Paris, 281p. (en anglais) ("L'art rupestre en Papouasie occidentale")

Aubert, M., O’Connor, S., McCulloch, M. T., Mortimer, G., Watchman, A., \& RicherLaflèche, M. 2007, Uranium-series dating rockart in East Timor. Journal of Archaeological Science, 34: 991-996. (en anglais) (“Datation de l'art rupestre à l’Uranium au Timor oriental”) doi:10.1016/j.jas.2006.09.017

Aubert, M., Brumm, A., Ramli, M., Sutikna, T., Saptomo, E. W., Hakim, B., Morwood, M. J., Van Den Bergh, G.D., Kinsley, L., \& Dosseto, A. 2014, Pleistocene cave art from Sulawesi, Indonesia. Nature, 514(7521): 223-227. (en anglais) (“Art rupestre pléistocène de Sulawesi, Indonésie”) doi:10.1038/nature13422

Auetrakulvit, P., Forestier, H., Khaokhiew, C., \& Zeitoun, V. 2012, New excavation at Moh Khiew site, Southern Thailand. In: Crossing Borders: Selected papers from the 13th International Conference of the European Association of Southeast Asian Archaeologists., Vol. 1 (Tjoa-Bonatz, M., Reinecke, A., Bonatz, D., Eds), NUS Press, Singapore: p. 60-71. (en anglais) ("Nouvelle fouille au site de Moh Khiew, sud de la Thaïlande”)

Balme, J., \& O’Connor, S. 2014, Chapter 13. Early Modern Humans in Island Southeast Asia and Sahul. Adaptative and creative societies with simple lithic industries. In: Southern Asia, Australia and the search form human origins (Dennell, R. \& Porr, M., Eds.), Cambridge University Press, Cambridge: p. 164-174. (en anglais) ("Chapitre 13. Les premiers humains modernes dans l'Asie du Sud-Est et Sahul. Des sociétés adaptatives et créatives avec des industries lithiques simples”) doi:10.1017/cbo9781139084741.013 
Barker, G. 2013, Rainforest Foraging and Farming in Island Southeast Asia: The Archaeology of the Niah Caves, Sarawak, volume 1. McDonald Institute Monographs, McDonald Institute for Archaeological Research, Cambridge, 410 p. (en anglais) ("Cueillette et agriculture dans la forêt tropicale dans l'île Asie du Sud-Est: L'archéologie des grottes de Niah, Sarawak”) doi:10.1002/arco.5066

Barker, G., Badang, D., Barton, H., Beavitt, P., Bird, M., Daly, P., Doherty, Ch., Gilbertson, D., Glover, I., Hunt, Ch., Manser, J., McLaren, S., Paz, V., Pyatt, B., Reynolds, T., Rose, J., Rushworth, G., \& Stephens, M. 2001, The Niah Cave Project: The second (2001) season of fieldwork. Sarawak Museum Journal, 56(77): 37-119. (en anglais) (“Le projet Niah Cave: la deuxième ( 2001) saison de travail sur le terrain”)

Barker, G., Lloyd-Smith, L., Barton, H., Cole, F., Hunt, C., Piper, P. J., Rabett, R., Paz, V., \& Szabo, K. 2011, Foraging-farming transitions at the Niah Caves, Sarawak, Borneo. Antiquity, 85(328): 492-509. (en anglais) ("Transitions de collecteurs à agricoles dans les grottes de Niah, Sarawak, Bornéo”) doi:10.1017/s0003598x00067909

Bartstra, G. J. 1976, Contribution to the study of the Palaeolithic Pacitanian Culture, Java, Indonesia. E. J. Brill, Leiden, 121 p. (en anglais) (“Contribution à l'étude de la culture paléolithique pacitanienne”)

Bartstra, G. J. 1982, Homo erectus: The search fo his artefacts. Current Anthropology, 23(3): 318-320. (en anglais) ("Homo erectus: la recherche de ses artefacts")

Bellwood, P. 1976, Archaeological research in Minahasa and Talaud Islands, northern Indonesia. Asian Perspectives, 19: 240-287. (en anglais) ("Recherches archéologiques dans les îles Minahasa et Talaud, nord de l'Indonésie”)

Bellwood, P. 1983, New perspectives on Indo-Malaysian prehistory. Bulletin of Indo-Pacific Prehistory Association, 4: 71-83. (en anglais) ("Nouvelles perspectives sur la préhistoire indo-malaisienne ”) doi:10.7152/bippa.v4i0.11210

Bellwood, P. 1984, Archaeological Research in in the Madai-Baturong region, Sabah. Bulletin of Indo-Pacific Prehistory Association, 5: 38-54. (en anglais) ("Recherches archéologiques dans la région de Madai-Baturong, Sabah”) doi:10.7152/bippa.v5i0.11223

Bellwood, P. 1985, Holocene flake and blade industrie of Wallacea and their predecessors. In: Recent advances in Indo-Pacific Prehistory (Misra, V.N., \& Bellwood, P., Eds.), E. J. Brill, Leiden: p. 197-206. (en anglais) (“Industrie à éclats et lames de l’Holocène à Wallacea et leurs prédécesseurs ”)

Bellwood, P. 1988, Archaeological research in south-eastern Sabah. In: Sabah Museum Monograph. Vol. 2 (Bellwood, P., Ed.), Sabah Museum and State Archives, Kota Kinabalu: p. 173-244. (en anglais) ("Recherches archéologiques dans le sud-est du Sabah")

Bellwood, P. 1989, Archaeological Investigations at Bukit Tengkorak and Segarong, Southeastern Sabah. Bulletin of Indo-Pacific Prehistory Association, 9: 122-162. (en anglais) ("Recherches archéologiques à Bukit Tengkorak et à Segarong, dans le sud-est du Sabah”) doi:10.7152/bippa.v9i0.11286

Bellwood, P. 1997. Prehistory of the Indo-Malaysian Archipelago (2nd edition). University of Hawaii Press, Honolulu, 373 p. (en anglais) ("Préhistoire de l'archipel indo-malais”) 
Bellwood, P. 2007, Prehistory of the Indo-Malaysian archipelago (revised edition). Australian National University, Canberra, 445 p. (en anglais) ("Préhistoire de l'archipel indo-malais”)

Bellwood, P., \& Koon, P. 1989, Lapita colonists leave boats unburned. The question of Lapita links with Island Southeast Asia. Antiquity, 63(240): 613-622. (en anglais) ("Les colons de Lapita laissent des bateaux non brûlés. La question des liens Lapita avec l'Asie du sud-est ”) doi:10.1017/s0003598x00076572

Boëda, E. 1993, Le débitage discoïde et le débitage Levallois récurrent centripète. Bulletin de la Société Préhistorique Française, 90(6): 392-404. (in French) ("Discoid debitage and recurrent centripetal Levallois debitage ”) doi:10.3406/bspf.1993.9669

Bordes, F. 1968, Le Paléolithique dans le monde. L’Univers des connaissances, Hachette, Paris, 256 p. (in French) ("The Paleolithic in the world")

Borel, A. 2012, Formes et fonctions au sein des industries lithiques de la fin du Pléistocène et du début de l'Holocène en Asie du Sud-Est, un nouvel apport à la compréhension des comportements humains, BAR International Series Vol. 2351, Archaeopress, Oxford, 384 p. (in French) ("Forms and Functions in Late Pleistocene and Early Holocene lithic industries in Southeast Asia, a new contribution to the understanding of human behavior")

Borel, A., Gaillard, C., Moncel, M. H., Sala, R., Pouydebat, E., Simanjuntak, T., \& Sémah, F. 2013, How to interpret informal flakes assemblages? Integrating morphological description, usewear and morphometric analysis gave better understanding of the behaviors of anatomically modern human from Song Terus (Indonesia), Journal of Anthropological Archaeology, 32(4): 630-646. (en anglais) (“Comment interpréter les assemblages d'éclats informels? L'intégration de la description morphologique, de l'utilisation et de l'analyse morphométrique a permis de mieux comprendre les comportements des humains anatomiquement modernes de Song Terus (Indonésie)”) doi:10.1016/j.jaa.2013.03.002

Bowler, J. M., Johnston, H., Olley, J. M., Prescott, J. R., Roberts, R. G., Shawcross, W., \& Spooner, N. A. 2003, New age for human occupation and climate change at Lake Mungo, Australia. Nature, 421(6925): 837-840. (en anglais) ("Nouvel âge pour l'occupation humaine et le changement climatique au lac Mungo, Australie") doi:10.1038/nature01383

Brandt, R. W. 1976, The Hoabinhian of Sumatra: Some remarks. In: Modern Quaternary Research in Southeast Asia, Vol. 2 (Bartstra, G. J., Casparie, W. A., Eds.), Balkema, Rotterdam: p. 49-52. (en anglais) ("Le Hoabinhian de Sumatra: quelques remarques”)

Brasseur, B., Sémah, F., Sémah, A. M., \& Djubiantono, T. 2011, Approche paléopédologique de l'environnement des hominidés fossiles du dôme de Sangiran (Java central, Indonésie). Quaternaire, 22: 13-34. (in French) ("Paleopedological approach to the environment of the fossil hominids of the Sangiran dome (Central Java, Indonesia)”) doi:10.4000/quaternaire.5815

Braüer, G., \& Mbua, E. 1992, Homo erectus features used in cladistics and their variability in Asian and African hominids. Journal of Human Evolution, 2: 79-108. (en anglais) (“Caractéristiques de l'Homo erectus utilisées en cladistique et leur variabilité chez les hominidés asiatiques et africains”) doi:10.1016/0047-2484(92)90032-5 
Bronson, B., \& Asmar, T. 1975, Prehistoric investigations at Tianko Panjang cave, Sumatra. Asian Perspectives, 18: 128-145. (en anglais) ("Recherches préhistoriques à la grotte Tianko Panjang, Sumatra”)

Bronson, B., \& Wisseman, N. 1974, An archaeological survey in Sumatra, 1973. Sumatra Research Bulletin, 4(1): 87-94. (en anglais) ("Une enquête archéologique à Sumatra, 1973”)

Brown, P., Sutikna, T., Morwood, M. J., Soejono, R. P., Jatmiko, Saptomo, E. W., \& Rokus Due Awe. 2004, A new small-bodied hominin from the Late Pleistocene of Flores, Indonesia. Nature, 431(7012): 1055-1061. (en anglais) (“Un nouvel homininé à petite corpulence du Pléistocène supérieur de Flores, Indonésie”) doi:10.1038/nature02999

Brumm, A., Aziz, F., Van den Bergh, G. D., Morwood, M.-J., Moore, M.-W., Kurniawan, I., Hobbs, D.-R., \& Fullagar, R. 2006, Early stone technology on Flores and its implications for Homo floresiensis. Nature, 441(7093): 624-628. (en anglais) ("La technologie de pierre au début sur Flores et ses implications pour Homo floresiensis”) doi:10.1038/nature04618

Brumm, A., Moore, M. W., Van den Bergh, G. D., Kurniawan, I., Morwood, M. J., \& Aziz, F. 2010, Stone technology at the Middle Pleistocene site of Mata Menge, Flores, Indonesia. Journal of Archaeological Science, 37(3): 451-473. (en anglais) ("La technologie de la pierre sur le site du Pléistocène moyen de Mata Menge”) doi:10.1016/j.jas.2009.09.012

Brumm, A. A., \& Moore, M. W. M. 2012, Biface distributions and the Movius Line: A Southeast Asian perspective. Australian Archaeology, 74(1): 34-46. (en anglais) ("La distribution du biface et la ligne Movius: une perspective de l'Asie du Sud-Est”) doi:10.1080/03122417.2012.11681933

Bulbeck, D. 2004, Divided in space, united in time: The Holocene prehistory of South Sulawesi. Indonesia. In: Modern Quaternary Research in Southeast Asia, Vol. 18 (Keates, S. G. \& Pasveer, J. M., Eds.), Balkema, Rotterdam: p.129-166. (en anglais) (“Divisé dans l'espace, uni dans le temps: la préhistoire holocène du Sud Sulawesi”)

Bulbeck, D. 2008, An integrated perspective on the Austronesian diaspora: The Switch from Cereal Agriculture to Maritime Foraging in the Colonisation of Island Southeast Asia. Australian Archaeology, 67(1): 31-51. (en anglais) (“Une perspective intégrée sur la diaspora austronésienne: le passage de l'agriculture céréalière à l'alimentation maritime dans la colonisation de l'Asie du sud-est”) doi:10.1080/03122417.2008.11681877

Bulbeck, D., Pasqua, M., \& Di Lello, A. 2000, Culture history of the Toalean of South Sulawesi, Indonesia. Asian Perspectives, 39(1): 71-108. (en anglais) ("Histoire de la culture du Toalean de Sud Sulawesi, en Indonésie”) doi:10.1353/asi.2000.0004

Bulbeck, D., Sumantri, I., \& Di Lello, A. 2004, Leang Sakapao I, a second dated Pleistocene site from South Sulawesi, In: Modern Quaternary Research in South-East Asia, Vol. 18 (Keates, S.G. \& Pasveer, J.-M., Eds.), Balkema, Rotterdam: p. 111-128. (en anglais) (“Leang Sakapao I, un deuxième site pléistocène daté du sud de Sulawesi”)

Chapman, V. 1981, An analysis of the artefact collections excavated by the AustralianIndonesian Archaeological expedition to Sulawesi, 1969. MA thesis, Australian National University, Canberra, 170 p. (en anglais) ("Une analyse des collections d'artefacts fouillés par l'expédition archéologique australo-indonésienne à Sulawesi, 1969”) 
Chapman, V. 1986, Inter-site variability in Southwest Sulawesi: Results of 1969 AustralianIndonesian Archaeological Expedition. Archaeology in Oceania, 21(1): 76-84. (en anglais) ("Variabilité inter-sites dans le sud-ouest de Sulawesi: résultats de l'expédition archéologique australo-indonésienne de 1969”) doi:10.1002/j.18344453.1986.tb00127.x

Chazine, J. M. 2005, Rock Art, Burials, and Habitations: Caves in East Kalimantan. Asian Perspectives, 44(1): 219-230. (en anglais) (“Art rupestre, sépultures et habitations: grottes au Kalimantan oriental”) doi:10.1353/asi.2005.0006

Chazine J. M. \& Ferrié, J. G. 2008, Recent archaeological discoveries in East Kalimantan, Indonesia. Bulletin of the Indo-Pacific Prehistory Association, 28: 16-22. (en anglais) ("Découvertes archéologiques récentes dans le Kalimantan oriental, Indonésie ”) doi:10.7152/bippa.v28i0.12011

Cherry, R. 1978, An analysis of a lithic industry of Buad Island, Samar. Philippine Quarterly of Culture and Society, 6: 3-80. (en anglais) ("Une analyse d'une industrie lithique de l'île de Buad, Samar”)

Chia, S., Yondri, L., \& Simanjuntak, H. T. 2008, L'origine des artefacts d'obsidienne de Gua Pawon, dago et Bukit Karsamanik à bandung, Indonésie. L'Anthropologie, 112(3): 448456. (en anglais) ("L'origine des objets en obsidienne de Gua Pawon, dago et Bukit Karsamanik à bandung, Indonésie”) doi:10.1016/j.anthro.2008.04.012

Christensen, O. A. 1975, A tanged blade from the New Guinea Highlands. Mankind, 10(1): 37-39. (en anglais) (“Une lame à pédoncule des Highlands de Nouvelle-Guinée”) doi:10.1111/j.1835-9310.1975.tb00910.x

Clarkson, C., Smith, M., Marwick, B., Fullagar, R., Wallis, L. A., Faulkner, P., Manne, T., Hayes, E., Roberts, R. G., Jacobs, Z., Carah, X., Lowe, K. M., Mattews, J., \& Florin, S. A. 2015, The archaeology, chronology and stratigraphy of Madjedbebe (Malakunanja II): A site in northern Australia with early occupation. Journal of Human Evolution, 83: 46-64. (en anglais) ("L'archéologie, la chronologie et la stratigraphie de Madjedbebe (Malakunanja II): Un site dans le nord de l'Australie avec une occupation précoce”) doi:10.1016/j.jhevol.2015.03.014

Datan, I. 1993, Archaeological Excavations at Gua Sireh (Serian) and Lubang Angin (Gunung Mulu National Park), Sarawak, Malaysia. Sarawak Museum Journal, Special Monograph 6: 192 p.. (en anglais) ("Fouilles archéologiques à Gua Sireh (Serian) et Lubang Angin (Parc national de Gunung Mulu), Sarawak, Malaisie”)

Datan, I., \& Bellwood, P. 1991, Recent research at Gua Sireh (Serian) and Lubang Angin (Gunung Mulu National Park), Sarawak. Bulletin of Indo-Pacific Prehistory Association, 10: 386-405. (en anglais) ("Recherches récentes à Gua Sireh (Serian) et Lubang Angin (Parc national de Gunung Mulu), Sarawak”) doi:10.7152/bippa.v10i0.11328

Dennell, R. 2009, The Palaeolithc settlement of Asia. Cambridge World Archaeology, Cambridge University Press, Cambridge, 548 p. (en anglais) (“L’occupation paléolithique d'Asie”)

Di Lello, A. 2002, A use-wear analysis of Toalian glossed stone artefacts from South Sulawesi, Indonesia. Indo-Pacific Prehistory Association Bulletin, 22: 45-50. (en anglais) ("Une analyse de l'usure des objets en pierre taillée du Toalian du sud de Sulawesi, en Indonésie”) doi:10.7152/bippa.v22i0.11804 
Dizon, E. Z. \& Pawlik, A. F. 2010, The Lower Palaeolithic record in the Philippines. Quaternary International, 223-224: 444-450. (en anglais) (“Le paléolithique inférieur aux Philippines”)

Fajari, N. M. E., \& Kusmartono, V. P. R. 2013, The excavation of Gua Payung, South Kalimantan, Indonesia. Journal of Indo-Pacific Archaeology, 33: 20-23. (en anglais) (“Les fouilles de Gua Payung, Kalimantan Sud, Indonésie”) doi:10.7152/jipa.v33i0.14511

Flenniken, J. J., \& White, J. P. 1985, Australian flaked stone tools: A technological perspective. Records of the Australian Museum, 36(3): 131-151. (en anglais) ("Les outils de pierre taillés australiens: une perspective technologique”) doi:10.3853/j.00671975.36.1985.342

Forestier, H. 1993, Le Clactonien : mise en application d'une nouvelle méthode de débitage s’inscrivant dans la variabilité des systèmes de production lithique du paléolithique ancien. Paléo, 5(1): 53-82. (in French) ("The Clactonian: Implementation of a new method of debitage falling within the variability of lithic production systems of the ancient Paleolithic ”) doi:10.3406/pal.1993.1104

Forestier, H. 1999, L'assemblage industriel de Song Keplek, Java Est : un nouveau regard sur l'outillage de l'homme moderne au début de l'Holocène en Indonésie. Bulletin de l'Ecole Française d'Extrême-Orient, 86(1): 129-159. (in French) (“The Song Keplek Industrial Assembly, East Java: A New Look at the Tooling of Modern Man in the Early Holocene in Indonesia ”) doi:10.3406/befeo.1999.3409

Forestier, H. 2000, De quelques chaînes opératoires lithiques en Asie du Sud-Est au Pléistocène supérieur final et au début de l'Holocène. L 'Anthropologie, 104(4): 531548. (in French) ("About some lithic chaînes opératoires in Southeast Asia during the late Upper Pleistocene and early Holocene ”) doi:10.1016/s0003-5521(00)80025-4

Forestier, H. 2007a, Les éclats du passé préhistorique de Sumatra : une longue histoire des techniques, Archipel, 74(1): 15-44. (in French) ("Flakes of the Prehistoric Past of Sumatra: A Long History of Techniques ”) doi:10.3406/arch.2007.3914

Forestier, H. 2007b, Ribuan Gunung, Ribuan Alat Batu : Prasejarah Song Keplek, Gunung Sewu, Jawa Timur. KPG-EFEO-IRD-Forum Jakarta Paris, Jakarta, 317 p. (en Indonésien ("Mille collines, Milles outils de pierre ”)

Forestier, H., \& Guillaud, D. 2013, A Limestone Outcrop as a Landmark of Prehistoric Settlement in the Manatuto Region (East Timor). In: Unearthing Southeast Asia's Past, Selected Papers from the 12th International Conference of the European Association of Southeast Asian Archaeologists. Vol. 1 (Klokke, M.-J. \& Degroot, V., Eds.), Leiden 2008, NUS Press, Singapore: p. 26-33. (en anglais) (“Un affleurement de calcaire comme un repère de l'établissement préhistorique dans la région de Manatuto (Timor oriental)”)

Forestier, H., Ceberti, V., \& Sophady, H. 2017, Le techno-complexe hoabinhien en Asie du Sud-est continentale : l'histoire d'un galet qui cache la forêt Journal of Lithic Studies, 4(2): 45 p.(in French) ("The Hoabinhian Techno-Complex in Continental Southeast Asia: The Story of a Pebble Hiding the Forest”) doi:10.2218/jls.v4i2.2545 
Forestier, H., Driwantoro, D., Guillaud, D., \& Budiman 2006, New data about Prehistoric chronology of South Sumatra. In: Indonesian Archaeology, Pr. R. P. Soejono Festschriift, Lipi, Jakarta: p. 177-192. (en anglais) ("Nouvelles données sur la chronologie préhistorique du sud de Sumatra”)

Forestier, H., Simanjuntak, H. T., Detroit, F., \& Zeitoun, V. 2010, Unité et diversité préhistorique entre Java et Sumatra. Archipel, 80: 19-44. (in French ("Prehistoric unity and diversity between Java and Sumatra”) doi:10.3406/arch.2010.4175

Forestier, H., Simanjuntak, H. T., \& Driwantoro, D. 2005a, Les premiers indices d'un faciès Acheuléen à Sumatra-sud, Indonésie? Dossiers d’Archéologie, 302: 16-17. (en anglais) ("The First Indications of an Acheulean facies at South-Sumatra, Indonesia?")

Forestier, H, Simanjuntak, H. T., Guillaud, D., Driwantoro, D., Wiradnyana, K., Siregar, D., Due Awe, R., \& Budiman 2005b, Le site de Tögi Ndrawa, île de Nias, Sumatra Nord : les premières traces d'une occupation hoabinhienne en grotte en Indonésie. C.R. Palevol 4(8): 727-733. (in French) ("The Tögi Ndrawa Site, Nias Island, North Sumatra: The first traces of a Hoabinhian cave occupation in Indonesia”) doi:10.1016/j.crpv.2005.08.004

Fox, R. B. 1970, The Tabon Caves. (Manila National Museum ed.) National Museum Monograph Vol. 1. The National Museum of Philippines, Manila, 197 p. (en anglais) (“Les Grottes de Tabon”)

Fullagar, R.L.K., Price, D.M., \& Head, L.M. 1996, Early human occupation of northern Australia : Archaeology and thermoluminescence dating of Jinmium rock-shelter, Northern territory. Antiquity, 70(270): 751-773. (en anglais) (“Occupation humaine ancienne du nord de l'Australie: Archéologie et datation par thermoluminescence de l'abri sous roche de Jinmium, territoire du Nord”) doi:10.1017/S0003598X00084040

Gay, M. 2010, Contribution à l'étude du peuplement préhistorique de l'Asie du Sud-Est: apport des méthodes de datation U/Th et 14C. Master 2 Université de Bordeaux, Bordeaux (in French) ("Contribution to the study of prehistoric settlement in Southeast Asia: contribution of U/Th and 14C dating methods")

Glover, I. C. 1972, Alfred Bühler's excavation in Timor: a re-evaluation. Art and Archaeology Research Papers, 2: 117-142. (en anglais) ("les fouilles d'Alfred Bühler au Timor: une réévaluation”)

Glover, I. C. 1976, Ulu Leang Cave, Maros: a preliminary sequence of post-Pleistocene cultural development in south Sulawesi. Archipel, 11(1): 113-54. (en anglais) ("Grotte d'Ulu Leang, Maros: une séquence préliminaire du développement culturel postPléistocène dans le sud de Sulawesi”) doi:10.3406/arch.1976.1271

Glover, I. C. 1977, The late stone age in Eastern Indonesia. World archaeology, 9(1): 42-61. (en anglais) ("L'âge de la pierre en Indonésie orientale") doi:10.1080/00438243.1977.9979684

Glover, I. C. 1979a, Report on a visit to archaeological sites near Medan, Sumatra, Utara, July 1975. Bulletin of Indo-pacific Prehistory Association, 1: 56-60. (en anglais) ("Rapport sur une visite de sites archéologiques près de Medan, Sumatra”) URL: https://journals.lib.washington.edu/index.php/BIPPA/article/view/11180/ 
Glover, I. C. 1979b, Survey and excavation in the Maros district, South Sulawesi, Indonesia: the 1975 field season. Bulletin of the Indo-Pacific Prehistory Association, 1: 61-102. (en anglais) ("Levé et fouilles dans le district de Maros, Sulawesi du Sud, Indonésie: la campagne de 1975”)

URL: https://journals.lib.washington.edu/index.php/BIPPA/article/view/11180/

Glover, I. C. 1979c, The Effects of Sink Action on Archaeological Deposits in Caves: An Indonesian Example. World Archaeology, 10(3): 302-317. (en anglais) ("Les effets de l'action de l'évier sur les dépôts archéologiques dans les grottes: un exemple indonésien”) doi:10.1080/00438243.1979.9979739

Glover, I. C. 1981, Leang Burung 2: an upper palaeolithic rock shelter in South Sulawesi, Indonesia. In: Modern Quaternary Research in South-East Asia, Vol. 6 (Bartstra, G.-J.

\& Casparie, W.-A., Eds.), Balkema, Rotterdam: p. 1-38. (en anglais) (“Leang Burung 2: un abri rocheux paléolithique supérieur dans le sud de Sulawesi, en Indonésie”)

Glover, I. C. 1986, Archaeology in Eastern Timor 1966-67. Terra Australia 11, Australian National University, Canberra, 241p. (en anglais) (“Archéologie au Timor Oriental 1966-67”)

Grenet, M., Sarel, J., Fauzy, R., Oktaviana, A.-A., Sugiyanto, B., Chazine, J.-M., \& Ricaut, F.-X. 2016, New insights on the late Pleistocene Holocene lithic industry in East Kalimantan (Borneo): The contribution of three rock shelter sites in the karstic area of the Mangkalihat peninsula. Quaternary International, 416: 126-150. (en anglais) ("Nouvelles perspectives sur l'industrie lithique du Pléistocène supérieur et de l’Holocène au Kalimantan oriental (Bornéo): Contribution de trois abris sous roche dans la zone karstique de la péninsule de Mangkalihat”) doi:10.1016/j.quaint.2015.11.044

Gunadi, H. 2006, Prehistoric dwelling caves in the area of Tepian Langsat, Kutai Timur, East Kalimantan. In: Indonesian Archaeology, Pr. R. P. Soejono Festschriift, Lipi, Jakarta, p. 201-210. (en anglais) (“Grottes d'habitation préhistoriques dans la région de Tepian Langsat, Kutai Timur, Kalimantan oriental”)

Haidle, M. N., \& Pawlik, A. F. 2009, Missing types: overcoming the typology dilemna of lithic archaeology in Southeast Asia. Bulletin of Indo-pacific Prehistory Association, 29: 2-5. (en anglais) ("Types manquants: surmonter le dilemme de la typologie dans l'archéologie lithique en Asie du Sud-Est”) doi:10.7152/bippa.v29i0.9470

Hakim, B. 2000, Mata panah bergiriri dari situs Pamangkulang Batua dan Batang Matasapo, Sulawesi Selatan. Walennae, 4: 36-41. (en indonésien) ("Prehistoric unity and diversity between Java and Sumatra”)

Hameau, S., Falguères, C., Bahain, J. J., Sémah, F., Sémah, A. M. \& Dolo, J. M. 2007, ESR dating in Song Terus cave (East Java, Indonesia). Quaternary Geochronology, 2(1-4): 398-402. (en anglais) ("Datation ESR de la grotte de Song Terus (Java Est, Indonésie)”) doi:10.1016/j.quageo.2006.04.011

Hanebuth, T. J. J., Voris, H. K., Yokoyama, Y., Saito, Y., \& Okuno, J. 2011, Formation and fate of sedimentary depocentres on Southeast Asia's Sunda Shelf over the past sea-level cycle and biogeographic implications. Earth-Science Reviews, 104(1-3): 92-110. (en anglais) ("Formation et devenir des dépôts sédimentaires sur le plateau de Sunda en Asie du Sud-Est sur le cycle marin et les implications biogéographiques”) doi:10.1016/j.earscirev.2010.09.006 
Harrisson, T. 1957, The Great Cave of Niah: A Preliminary Report on Bornean Prehistory. Man, 57: 161-166. (en anglais) (“La grande grotte de Niah : rapport préliminaire sur la préhistoire de Bornéo”) doi:10.2307/2795279

Harrisson, T. 1959, New Archaeological and Ethnological Results from Niah Caves, Sarawak. Man, 59: 1-8. (en anglais) ("Nouveaux résultats archéologiques et ethnologiques des grottes de Niah, Sarawak”) doi:10.2307/2796008

Hartono, H. M.-S. 1961, Geological investigations at Olabula, Flores. Unpublished report, Djawatan Geologi, Bandung, Indonesia, 1-41. (en anglais) ("Recherches géologiques à Olabula, Flores”)

Hooijer, D. A. 1957, A Stegodon from Flores. Treubia, 24: 119-129. (en anglais) (“Un Stegodon de Flores”)

Hooijer, D. A. 1964, On two milk molars of a pygmy stegodont from Ola Bula. Flores. Bulletin of the Geological Survey of Indonesia, 1: 49-51. (en anglais) ("Sur deux molaires de lait d'un stegodont pygmée d'Ola Bula. Flores”)

Hooijer, D. A. 1969, Indonesian prehistoric tools. A catalogue of the Houbolt Collection. R. J. Brill, Leiden, 175 p. (en anglais) (“Outils préhistoriques indonésiens”)

Hooijer, D. A. 1972, Stegodon trigonocephalus florensis Hooijer and Stegodon timorensis Sartono from the Pleistocene of Flores and Timor. I \& II. Proceedings van de Koninklijke Nederlandse Akademie van Wetenschappen, Serie B, 75: 12-33. (en anglais) ("Stegodon trigonocephalus florensis Hooijer et Stegodon timorensis Sartono du Pléistocène de Flores et du Timor. I \& II”)

Ingicco, T. 2012, Les primates quaternaires de Song terus (Java Est, Indonésie): implications paléobiogéographiques et archéozoologiques pour l’Asie du Sud-Est. Bulletins et mémoires de la Société d'Anthropologie de Paris, 24(3-4): 199-203. (en anglais) (“Les primates quaternaires de Song terus (Java Est, Indonésie): implications paléobiogéographiques et archéozoologiques pour l'Asie du Sud-Est”) doi:10.1007/s13219-012-0067-1

Jatmiko, Nasruddin, \& Sugiyanto, B. 2004, Eksplorasi situs gua dan ceruk hunian prasejarah di Pegunungan Marang, Laporan Penilitian Arkeologi, Puslit Arkenas, Jakarta, 38 p. (en indonésien) ("Exploration des sites en grotte et des cavités préhistoriques dans les montagnes de Marang”)

Keates, S. G. 2004, Notes on the palaeolithic finds from Walanae valley, southwest Sulawesi, in the context of the Late Pleistocene of Island Southeast Asia. In: Modern Quaternary Research in South-East Asia, Vol. 18, (Keates, S. G., Pasveer \& J. M., Eds.), Balkema, Rotterdam: p. 95-110. (en anglais) ("Notes sur les découvertes paléolithiques de la vallée de Walanae, dans le sud-ouest de Sulawesi, dans le contexte du Pléistocène supérieur de l'Asie du Sud-Est”)

Keates, S. G., \& Bartstra, G. J. 2001, Observations on Cabengian and Pacitanian artefacts from the island Southeast Asia. Quartär, Band 51/52: 9-32. (en anglais) (“Observations sur les artéfacts cabengiens et pacitaniens de l'île Asie du Sud-Est”)

Kim, J. Y., \& Lee, Y. J. 2006, La stratigraphie du Pléistocène supérieur et le Paléolithique de Corée. L'Anthropologie, 110(2): 119-138. (in French) (“Upper Pleistocene and Paleolithic stratigraphy of Korea ”) doi:10.1016/j.anthro.2006.03.010

Kristianasen, W. (2010, novembre) Indonésie, musulmans contre islamistes. Le Monde Diplomatique, 680, pp. 16-17. (in French) (“Indonesia, muslims against islamists”) 
Lee, Y. J., \& Yun, Y. H. 1993, Tanged point and micro-blade core from Suyanggae, Korea. In: The origin and dispersal of microblade industry in Northern Eurasia. (Kimura, H., Ed.), Sapporo University, Sapporo, Japan: p. 52-69. (en Anglais) ("Pointe à pédoncule et nucléus à lamelles de Suyanggae, Corée”)

Liu, W., Jina, C. Z., Zhang, Y. Q., Cai, Y. J., Xing, S., Wu, X. J., Cheng, H., Edwards, R., Pan, W. S., Qin, D. G., An, Z. S., Trinkaus, E., \& Wu, X. Z. 2010, Human remains from Zhirendong, South China, and modern human emergence in East Asia. Proceedings of the National Academy of Sciences, 107(45): 19201-19206. (en anglais) ("Restes humains de Zhirendong, Chine du Sud, et l'émergence humaine moderne en Asie de l'Est") doi:10.1073/pnas.1014386107

Lyras, G. A., Dermitzakis, M. D., Van der Geer, A. A. E., Van der Geer, S. B., \& De Vos, J. 2009, The origin of Homo floresiensis and its relation to evolutionary processes under isolation. Anthropological Science, 117: 33-43. (en anglais) ("L'origine de Homo floresiensis et sa relation avec les processus évolutifs sous l'isolement”) doi:10.1537/ase.080411

Mahirta. 2009, Stone technology and the chronology of human occupation on Rote, Sawu and Timor, Nusa Tenggara Timur, Indonesia. Bulletin of Indo-Pacific Prehistory Association, 29: 101-108. (en anglais) ("La technologie de la pierre et la chronologie de l'occupation humaine sur Rote, Sawu et Timor, Nusa Tenggara Timur, Indonésie”) doi:10.7152/bippa.v29i0.9483

Majid, Z. 1982, The West Mouth, Niah, in the Prehistory of Southeast Asia. Sarawak Museum Journal, 31(52): 1-200. (en anglais) ("The West Mouth, Niah, dans la Préhistoire de l'Asie du Sud-Est”)

Maringer, J., \& Verhoeven, T. 1970a, Note on some stone artifacts in the National Archaeological Institute of Indonesia at Djakarta, collected from the Stegodon-fossil Bed at Boaleza in Flores. Anthropos, 65: 638-639. (en anglais) ("Note sur quelques artefacts en pierre dans l'Institut archéologique national de l'Indonésie à Djakarta, recueillis du lit fossile de Stegodon à Boaleza à Flores”)

Maringer, J., \& Verhoeven, T. 1970b, Die Steinarterfakte aus der Stegodon-Fossilschicht von Mengeruda auf Flores, Indonesien. Anthropos, 65: 229-247. (en allemand) ("Les outils de pierre et les restes fossils de Stegodon de Mengeruda de Flores”)

Maringer, J., \& Verhoeven, T. 1970c, Die Oberflächenfunde aus dem Fossilgebiet von Mengeruda und Olabula auf Flores, Indonesien. Anthropos, 65: 530-546. (en allemand) (“Les outils de pierre et les restes fossils de Stegodon de Mengeruda de Flores”)

Maringer, J., \& Verhoeven, T. 1975, Die Oberflächenfunde von Marokoak auf Flores, Indonesien. Anthropos, 70: 97-104. (en allemand) (“Les restes de surface de Marokoak de Flores”)

McCarty, F. D. 1976, Australian aboriginal stone implements. ( $2^{\text {nd }}$ edition). The Australian Museum Trust, Sydney, 107 p. (en anglais) ("Les outils de pierre aborigènes australiens")

McKinnon, E. E. 1990, The Hoabinhian in the Wampu/Lau Biang valley of Northeastern Sumatra: An update. Bulletin of Indo-Pacific Prehistory Association, 10: 132-142. (en anglais) ("Le Hoabinhian dans la vallée Wampu / Lau Biang du nord-est de Sumatra: une mise à jour”) doi:10.7152/bippa.v10i0.11302 
Mijares, A. S., Detroit, F., Piper, F., Bellwood, P., Grün, R., Aubert, M., Cuevas, N., De Leon, A., \& Dizon, E. 2010, New evidence for a 67,000 year old human presence at Callao cave, Luzon, Philippines. Journal of Human Evolution, 59(1): 123-132. (en anglais) ("De nouvelles preuves pour une présence humaine de 67,000 ans dans la grotte Callao, Luzon, Philippines”) doi:10.1016/j.jhevol.2010.04.008

Moigne, A. M., Rokhus Due Awe., Sémah, F., \& Sémah, A. M. 2004, The Cervids from the Ngebung Site ("Kabuh" Series, Sangiran Dome, Central Java) and Their Biostratigraphical Significance. In: Modern Quaternary Research in Southeast Asia. Vol. 18, (Keates, S.G. \& Pasveer, J. M., Eds.), Balkema, Rotterdam: p. 31-44. (en anglais) ("Les Cervidés du site de Ngebung (série «Kabuh», Sangiran Dome, Java central) et leur importance biostratigraphique”)

Moore, M. W., \& Brumm, A. 2007, Stone artifacts and hominins in island Southeast Asia: New insights from Flores, eastern Indonesia. Journal of Human Evolution, 52(1): 85102. (en anglais) ("Artefacts en pierre et homininés en Asie du Sud-Est: nouvelles données de Flores, Indonésie orientale”) doi:10.1016/j.jhevol.2006.08.002

Moore, M. W., Sutikna, T., Jatmiko, Morwood, M. J., \& Brumm, A. 2009, Continuities in stone flaking technology at Liang Bua, Flores, Indonesia. Journal of Human Evolution, 57(5): 503-526. (en anglais) (“Continuités dans la technologie de la pierre taillée à Liang Bua, Flores, Indonésie”) doi:10.1016/j.jhevol.2008.10.006

Morwood, M. J., Aziz, F., Van den Bergh, G. D., Sondaar, P. Y., \& de Vos, J. 1997, Stone artefacts from the 1994 excavation at Mata Menge, West central Flores, Indonesia. Australian Archaeology, 44(1): 26-34. (en anglais) (“Objets en pierre de la fouille de 1994 à Mata Menge, Flores central ouest, Indonésie”) doi:10.1080/03122417.1997.11681587

Morwood, M. J., Soejono, R.P., Roberts, R. G., Sutikna, T., Turney, C. S. M., Westaway, K. E., Rink, J., Zhao, J. X., Van den Bergh, G. D., Rokus Awe Due, Hobbs, D. R., Moore, M. W., Bird, M. I., \& Fifield' L. K. 2004, Archaeology and age of a new hominin from Flores in eastern Indonesia. Nature, 431(7012): 1087-1091. (en anglais) (“Archéologie et âge d'un nouvel homininé de Flores dans l'est de l'Indonésie”) doi:10.1038/nature02956

Morwood, M. J., O’Sullivan, P. B., Aziz, F., \& Raza, A. 1998, Fission-track ages of stone tools and fossils on the east Indonesian island of Flores. Nature, 392: 173-176. (en anglais) (“Age des outils en pierre et des fossiles sur l'île indonésienne de Flores”) doi:10.1038/32401

Morwood, M. J., Aziz, F., O’Sullivan, P., Nasruddin, Hobbs, D. R., \& Raza, A. 1999, Archaeological and paleontological research in central Flores, east Indonesia: results of fieldwork 1997-98. Antiquity, 73(280): 273-286. (en anglais) ("La recherche archéologique et paléontologique dans le centre de Flores, à l'est de l'Indonésie: résultats du travail de terrain 1997-98”) doi:10.1017/s0003598x00088244

Morwood, M. J., O’Sullivan, P., Susanto, E. E., \& Aziz, F. 2003, Revised age for Modjokerto1, an Early Homo erectus cranium for East Java. Australian Archaeology, 57: 1-4. (en anglais) (“Âge révisé pour Modjokerto1, un crâne de l'Homo erectus ancien pour le Java oriental”) doi:10.1080/03122417.2003.11681757 
Morwood, M.J., Brown, P., Jatmiko, Sutikna, T., Wahyu Saptomo, E., Westaway, K.E., Awe Due, R., Roberts, R.G., Maeda, T., Wasisto, S., \& Djubiantono, T. 2005, Further evidence for small-bodied hominins from the Late Pleistocene of Flores, Indonesia. Nature, 437(7061): 1012-1017. (en anglais) ("Preuve supplémentaire pour les homininés de petite taille du Pléistocène supérieur de Flores, Indonésie ”) doi:10.1038/nature04022

Mulvaney, D. J. 1969, The Prehistory of Australia. Thames and Hudson, London, 276 p. (en anglais) ("La Préhistoire de l'Australie")

Mulvaney, J., \& Soejono, R. P. 1970, The Australian-Indonesian archaeological expedition to Sulawesi. Asian Perspectives, 13: 163-177. (en anglais) (“L'expédition archéologique australo-indonésienne à Sulawesi”)

Mulvaney, J., \& Soejono, R. P. 1971, Archaeology in Sulawesi, Indonesia. Antiquity, 45(177): 26-33. (en anglais) (“Archéologie à Sulawesi, Indonésie”) doi:10.1017/s0003598x00069015

Mulvaney, D. J., \& Kamminga, J. 1999, Prehistory of Australia. Simthsonian Institution Press, Washington D.C., 480 p. (en anglais) ("Préhistoire de l'Australie”)

Nelson, S. M. 1993, The archaeology of Korea. Cambridge World Archaeology series. Cambridge University Press, 324 p. (en anglais) ("L'archéologie de la Corée”)

O’Connell, C. A., \& DeSilva, J. M. 2013, Modjokerto revisited: Evidence for an intermediate pattern of brain growth in Homo erectus. Journal of Human Evolution, 65: 156-161. (en anglais) ("Modjokerto revisité: preuve d'un modèle intermédiaire de croissance cérébrale chez Homo erectus”) doi:10.1016/j.hevol.2013.04.007

O’Connell, J. F., \& Allen, J. 2004, Dating the colonization of Sahul (Pleistocene AustraliaNew Guinea): a review of recent research. Journal of Archaeological Science, 31(6): 835-853. (en anglais) ("Datation de la colonisation de Sahul (Pléistocène AustralieNouvelle-Guinée): un examen des recherches récentes”) doi:10.1016/j.jas.2003.11.005

O’Connor, S. 2003, Nine new painted rock Art sites in East Timor in the context of the Western Pacific region. Asian Perspectives, 42(1): 96-128. (en anglais) (“Neuf nouveaux sites d'art rupestre peints au Timor oriental dans le contexte de la région du Pacifique occidental”) doi:10.1353/asi.2003.0028

O’Connor, S. 2007, New evidence from East Timor contributes to our understanding of earliest modern human colonisation east of the Sunda Shelf. Antiquity, 81(313): 523535. (en anglais) ("De nouvelles preuves provenant du Timor oriental contribuent à notre compréhension de la première colonisation humaine moderne à l'est du plateau de Sahul”) doi:10.1017/s0003598x00095569

O’Connor, S., \& Veth, P. 2005, Early Holocene shell fishhooks from Lene Hara Cave, East Timor, establish complex fishing technology was in use in island Southeast Asia five thousand years before Austronesian settlement, Antiquity, 79(304): 249-256. (en anglais) ("Des hameçons en coquillages de l'Holocène de la grotte de Lene Hara, Timor oriental, établissent que la technologie de pêche complexe était utilisé dans l'île Asie du Sud-Est cinq mille ans avant la colonisation austronésienne”) doi:10.1017/s0003598x0011405x 
O’Connor, S., Spriggs, M., \& Veth, P. 2002, Excavation of Lene Hara Cave establishes occupation in East Timor at least 30.000-35.000 years ago. Antiquity, 76(291): 45-49. (en anglais) ("La fouille des grottes de Lene Hara établit l'occupation au Timor oriental au moins 30.000-35.000 ans”) doi:10.1017/s0003598x0008978x

Olsen, S. L., \& Glover, I. C. 2004, The bone industry of Ulu Leang I and Leang Burung I rockshelters, Sulawesi, Indonesia, in its regional context. In: Modern Quaternary Research in South-East Asia, Vol. 18, (Pasveer, J. M., Ed.), Balkema, Rotterdam: p. 273-299. (en anglais) (“L'industrie de l'os de Ulu Leang I et Leang Burung I rockshelters, Sulawesi, en Indonésie, dans son contexte régional”)

O’Sullivan, P. B., Morwood, M. J., Hobbs, D., Aziz, F., SumintoSitumorang, M., Raza, A., \& Maas, R. 2001, Archaeological implications of the geology and chronology of the So'a Basin, Flores, Indonesia. Geology, 29(7): 607-610. (en anglais) ("Les implications archéologiques de la géologie et la chronologie du bassin de So'a , Flores, Indonésie”) doi:10.1130/0091-7613(2001)029\%3C0607:aiotga\%3E2.0.co;2

Pasqua, M., \& Bulbeck, D. 1998, A technological interpretation of the Toalean, South Sulawesi. In: Modern Quaternary Research in South-East Asia, Vol. 15, (Bartstra, G.J., Ed.), Balkema, Rotterdam: p. 211-232. (en anglais) ("Une interprétation technologique du Toalean, South Sulawesi”)

Pawlik, A. 2001, Acheulean in Nueva Ecija? Hukay, 4: 1-22. (en anglais) (“Acheuléen à Nueva Ecija?”)

Pawlik, A. 2004, The Palaeolithic site of Arubo 1 in Central Luzon, Philippines. Indo-Pacific Prehistory Association Bulletin, 24: 3-12. (en anglais) ("Le site paléolithique d'Arubo 1 au centre de Luzon, aux Philippines”) doi:10.7152/bippa.v24i0.11866

Pawlik, A., \& Ronquillo, W. P. 2003, The Palaeolithic in the Philippines. Lithic Technology, 28(2): 79-93. (en anglais) ("Le Paléolithique aux Philippines”) doi:10.1080/01977261.2003.11721004

Plutniak, S. Oktaviana, A. A., Sugiyanto, B., Chazine, J. M., \& Ricaut, F. X. 2014, New Ceramic Data from East Kalimantan: The cord-marked and red-slipped sherds of Liang Abu's layer 2 and Kalimantan's pottery chronology. Journal of Pacific Archaeology, 5(1): 90-99. (en anglais) (“Nouvelles données céramiques du Kalimantan oriental: Les tessons de la couche 2 de Liang Abu et la chronologie de la poterie de Kalimantan”)

Polanski, J.M., Marsh, H.E., \& Maddux, S.D. 2016, Dental size reduction in Indonesian Homo erectus: Implications for the PU-198 premolar and the appearance of Homo sapiens on Java. Journal of human evolution, 90: 49-54. (en anglais) ("Réduction de la taille dentaire chez l'Homo erectus indonésien: Implications pour la prémolaire PU-198 et l'apparition de l'Homo sapiens sur Java”) doi:10.1016/j.jhevol.2015.09.008

Presland, G. 1980, Continuity in Indonesian lithic traditions. The Artefact, 5: 19-46. (en anglais) (“Continuité dans les traditions lithiques indonésiennes”)

Rabett, R. 2012. Human Adaptation in Asian Palaeolithic: Hominin Dispersal and Behaviour during the Late Quaternary. Cambridge University Press, Cambridge, 372 p. (en anglais) ("Adaptation humaine au paléolithique asiatique: dispersion et comportement de l'homininé au cours du quaternaire tardif”) doi:10.1017/CBO9781139087582 
Ricaut, F. X., Sugiyanto, B., Ferrié, J. G., Setiawan, P., Sarel, J., Oktaviana, A. A., Guerreiro, A., Amuranto, B., Grenet, M., Plutniak, S., Voeltzel, B., Chazine, J. M., \& Djubiantono, D. 2011, Prehistoric occupation at the rock shelter of Liang Abu, Eastern Borneo. Antiquity, 85: 329. (en anglais) (“Occupation préhistorique à l'abri de la roche de Liang Abu, Bornéo Est”)

Ricaut, F. X., Ferrié, J. G., Sarel, J., Guerreiro, A., Plutniak, S., \& Chazine, J. M. 2012, Fouilles archéologiques Franco-Indonésienne à Kalimantan Est. Unpublished interim report for the Ministère des Affaires Etrangères (Ministry of Foreign and European Affairs), Paris, 126 p. (in French) ("French-Indonesian archaeological excavation in East Kalimantan”)

Ricaut, F. X., Ferrié, J. G., Grenet, M, Guerreiro, A., Plutniak, S., \& Chazine, J. M. 2013, Fouilles archéologiques Franco-Indonésienne à Kalimantan Est. Unpublished interim report to the Ministère des Affaires Etrangères (Ministry of Foreign and European Affairs), Paris, 79 p. (in French) ("French-Indonesian archaeological excavation in East Kalimantan”)

Reyes-Centeno, H. 2016. Out of Africa and into Asia: Fossil and genetic evidence on modern human origins and dispersals. Quaternary International, 416: 249-262. (en anglais) ("Hors d'Afrique et en Asie: Fossiles et preuves génétiques sur les origines et les dispersions humaines modernes”) doi:10.1016/j.quaint.2015.11.063

Roberts, R., Bird, M., Olley, J., Galbraith, R., Lawson, E., Laslett, G., Yoshida, H., Jones, R., Fullagar, R., Jacobsen, G. \& Hua, Q. 1998, Optical and radiocarbon dating at Jinmium rock shelter in northern Australia. Nature, 393: 358-362. (en anglais) ("Datation optique et radiocarbone à l'abri sous roche de Jinmium dans le nord de l'Australie”) doi:10.1038/30718

Roberts, R. G., Jones, R., \& Smith, M. A. 1990, Thermoluminescence dating of a 50,000 year old human occupation site in Northern Australia. Nature, 345(6271): 153-156. (en anglais) ("Datation par Thermoluminescence d'un site d'occupation humaine de 50,000 ans dans le nord de l'Australie”) doi:10.1038/345153a0

Roberts, R.G., Jones, R., Spooner, N.A., Head, M.J., Murray, A.S., \& Smith, M.A. 1994, The human colonisation of Australia: optical dates of 53,000 and 60,000 years brackets human arrival at Deaf Adder Gorge, Northern Territory. Quaternary Geochronology (Quaternary Science Review), 13: 575-583. (en anglais) ("La colonisation humaine de l'Australie: des dates optiques de 53,000 et 60,000 ans datent l'arrivée des humains à Deaf Adder Gorge, Territoire du Nord ”) doi:10.1016/0277-3791(94)90080-9

Saidin, M., \& Jeffrey, A. 2014, Sumbangan Sabah Kepada Arkeologi Asia Tenggara Hasil Penyelidikan 20 Tahun (1993-2013). vol. 12. Monograf Muzium Sabah, Kota Kinabalu, Sabah. (en malais) ("French-Indonesian archaeological excavation in East Kalimantan")

Sathiamunthy, E., \& Voris, H. K. 2006, Maps of Holocene sea level transgression and submerged lakes on the Sunda Shelf. The Natural History Journal of Chulalongkorn University, 2: 1-43 (en anglais) ("Cartes de la transgression holocène du niveau de la mer et des lacs submergés sur le plateau de Sahul”) 
Sémah, F. 2001, La position stratigraphique du site de Ngebung 2 (dôme de Sangiran, Java Central, Indonésie). In: Origine des peuplements et chronologie des cultures paléolithiques du Sud-est asiatique, (Sémah, F., Falguères, C., Grimaud-Hervé, D., \& Sémah, A. M., Eds.), Semenanjung-Artcom', Paris: p. 299-329. (in French) (“The Stratigraphic Position of the Ngebung 2 Site (Sangiran Dome, Central Java, Indonesia)")

Sémah, F., Sémah, A. M., \& Djubiantono, T. 1992, Did they also make stone tools? Journal of Human Evolution, 23(5): 439-446. (en anglais) (“Ont-ils également fait des outils en pierre?”) doi:10.1016/0047-2484(92)90092-n

Sémah, F., Sémah, A. M., \& Simanjuntak, H. T. 2002, More than a million years of human occupation in insular Southeast Asia: The early archaeological record of Eastern and Central Java. In: Under the Canopy: the archaeology of tropical rainforests. (Mercader, J., Ed.), Rutgers University Press, Rutgers: p. 161-190. (en anglais) ("Plus d'un million d'années d'occupation humaine dans l'Asie du sud-est insulaire: Les premiers enregistrements archéologiques de l'est et du centre de Java”)

Sémah, A. M., Sémah, F., Moudrikah, R., Frölich, F., \& Djubiantono, T. 2004a, A late Pleistocene and Holocene sedimentary record in Central Java and its paleoclimatic significance. In: Modern Quaternary Research in Southeast Asia, Vol. 18, (Keates, S.G. \& Pasveer, J.-M., Eds.), Balkema, Rotterdam: p. 63-88. (en anglais) (“Un enregistrement sédimentaire pléistocène tardif et Holocène dans le centre de Java et sa signification paléoclimatique”)

Sémah, F., Sémah, A. M., Falguères, C., Détroit, F., Simanjuntak, H. T., Moigne, A. M., Gallet, X., \& Hameau, S. 2004b, The significance of the Punung karstic area (Eastern Java) for the chronology of the Javanese Palaeolithic, with special reference to the Song Terus cave. In: Modern Quaternary Research in Southeast Asia, Vol. 18, (Keates, S. G., \& Pasveer, J. M., Eds.), Balkema, Rotterdam: p. 45-61. (en anglais) (“L'importance de la zone karstique de Punung (Java oriental) pour la chronologie du Paléolithique javanais, avec une référence particulière à la grotte Song Terus”)

Sémah, F., Sémah, A. M., \& Widianto, H. 2014a, Insular Southeast Asia at the interface of continent-archipelago: Geography and chronology. In: Encyclopedia of Global Archaeology. (Smith, C., Ed.), Springer, New York: p. 3890-3904. (en anglais) ("L’Asie du Sud-Est insulaire à l'interface de l'archipel-continent: Géographie et chronologie”) doi:10.1007/978-1-4419-0465-2-1906

Sémah, F., Simanjuntak, H. T., Dizon, B., \& Sémah, A. M. 2014b, Insular Southeast Asia in the Lower Paleolithic. In: Encyclopedia of Global Archaeology. (Smith, C., Ed.), Springer, New York: p. 3904-3918. (en anglais) (“L’Asie du sud-est insulaire dans le Paléolithique inférieur”) doi:10.1007/978-1-4419-0465-2-1907

Seong, C. 2008, Tanged point, microblades and Late Palaeolithic hunting in Korea. Antiquity, 82(318): 871-883. (en anglais) ("Pointe à pédoncule, lamelles et la chasse au Paléolithique supérieur en Corée”) doi:10.1017/s0003598x00097647

Setiagama, F. K. 2006, L'industrie osseuse de l'horizon Keplek, Holocène de la grotte de Song Terus, Punung, Java Est (Indonésie). Master au Muséum National d'Histoire Naturelle (Master's thesis IMQP), Paris, France, 157 p. (in French) ("The Keplek Horizon Bone Industry, Holocene of Song Terus Cave, Punung, East Java, Indonesia”) 
Shen, G., \& Michel, V. 2007. Position chronologique des sites de l'Homme moderne en Chine d'après la datation UTh. L'anthropologie, 111: 157-65. (in French) ("Chronological position of modern human sites in China according to UTh dating”) doi:10.1016/j.anthro.2007.02.001

Shen, G., Wang, W., Wang, Q., Zhoa, J., \& Collerson, K. 2002, U-series dating of Liujiang hominid site in Guanxi, southern China. Journal of Human Evolution, 43(6): 817-829. (en anglais) ("Datation Uth du site hominidé de Liujiang à Guanxi, sud de la Chine”) doi:10.1006/jhev.2002.0601

Simanjuntak, H. T. 1995, Mésolithique en Indonésie : une hétérogénéité culturelle. L'Anthropologie, 99(4): 626-636. (in French) ("Mesolithic in Indonesia: cultural heterogeneity")

Simanjuntak, H. T. 2002, Gunung Sewu in prehistoric time. (Simanjuntak, H. T., Ed.). Gajah Mada University Press, Yogyakarta, 220 p. (en anglais) (“Gunung Sewu à l'époque préhistorique”)

Simanjuntak, H. T. 2006, Indonesia-Southeast Asia: Climates, settlements, and cultures in Late Pleistocene. C.R. Palevol, 5(1-2): 371-379. (en anglais) (“Indonésie-Asie du SudEst: Climats, peuplements et cultures au Pléistocène supérieur”) doi:10.1016/j.crpv.2005.10.005

Simanjuntak, H. T., \& Forestier, H. 2004, Research progress on the Neolithic in Indonesia: Special reference to the Pondok Silabe cave, South Sumatra. In: Southeast Asian Archaeology. (Paz, V., Ed.), Wilhelm G. Solheim II Festschriift. The University of The Philippines Press, Diliman, Quezon City, Manila: p. 104-118. (en anglais) ("Progrès de la recherche sur le néolithique en Indonésie: référence spéciale à la grotte de Pondok Silabe, au sud de Sumatra”)

Simanjuntak, H. T., \& Forestier, H. 2008, Handaxe in Indonesia. A question on the Movius Line. Human Evolution, 23(1-2): 97-107. (en anglais) (“Bifaces en Indonésie. Une question sur la ligne Movius”)

Simanjuntak, H. T., \& Forestier, H. 2009, Once upon a time in South Sumatra: the Acheulean stone tools of the Ogan River? In: Proceedings of the International Seminar on Sharing our Archaeological Heritage, Penang, Malaysia. (Saidin M., \& Razak, K. Ab., Eds.), Warisan Johor, Penang: p. 233-241. (en anglais) ("Il était une fois dans le sud de Sumatra: les outils de pierre acheuléens de la rivière Ogan?”)

Simanjuntak, H. T., Sémah, F., \& Gaillard, C. 2010, The Palaeolithic in Indonesia: Nature and chronology. Quaternary International, 223-224: 418-421. (en anglais) (“Le Paléolithique en Indonésie: Nature et chronologie”) doi:10.1016/j.quaint.2009.07.022

Sinha, P., \& Glover, I. C. 1984, Changes in stone tool use in Southeast Asia, 10000 years ago: a microwear analysis of flakes with use gloss from Leang Burung 2 and Ulu Leang caves, Sulawesi, Indonesia. In: Modern Quaternary Research in Southeast Asia, Vol. 8, (Pasveer, J. M., Ed.), Balkema, Rotterdam: p. 137-191. (en anglais) (“Évolution de l'utilisation des outils en pierre en Asie du Sud-Est, il y a 10000 ans: analyse tracéologique des éclats avec des polis d'utilisation des grottes Leang Burung 2 et Ulu Leang, Sulawesi, Indonésie”)

Soejono, R.P. 1979, The significance of excavations at Gilimanuk (Bali). In: Early South East Asia (Smith, R.B. \& Watson, W, Eds.), Oxford University Press, New York, p. 185198. (en anglais) ("La signification des fouilles a Gilimanuk (Bali)") 
Soejono, R. P. 1984a, Prehistoric Indonesia. In: Prehistoric Indonesia: a reader. (Van de Velde, P., Ed.), Floris Publication, Dordrecht, Holland \& Cinnaminson, USA: p. 52-78. (en anglais) (“L’Indonésie préhistorique”)

Soejono, R. P. 1984b, Important prehistoric discoveries in Indonesia. Jurnal Arkeologi Malaysia, 4: 16-22. (en anglais) ("Importantes découvertes préhistoriques en Indonésie”)

Soejono, R. P. 1991, Paleolitik di Indonesia. Seminar Peringatan 100 tahun penemuan Pithecanthropus. Puslit Arkenas. Museum Nasional, Cedust, Jakarta. (en indonésien) ("French-Indonesian archaeological excavation in East Kalimantan")

Sondaar, P. Y., Van den Bergh, G. D., Mubroto, B., Aziz, F., de Vos, J., \& Batu, U. L. 1994. Middle Pleistocene faunal turn-over and colonisation of Flores (Indonesia) by Homo erectus. Comptes Rendus de l'Académie des Sciences, 319: 1255-1262. (en anglais) ("Le turn-over faunique du Pléistocène moyen et la colonisation de Flores (Indonésie) par Homo erectus”)

Spriggs, M., Reepmeyer, C., Anggraeni, Lape, P., Neri, L., Ronquillo, W. P., Simanjuntak, H. T., Summerhayes, G., Tanudirjo, D., \& Tiauzon, A. 2011. Obsidian sources and distribution systems in island Southeast Asia: a review of previous research. Journal of Archaeological Science, 38(11): 2873-2881. (en anglais) (“Sources d'obsidienne et systèmes de distribution en Asie du Sud-Est: un examen des recherches antérieures”) doi:10.1016/j.jas.2011.06.015

Stone, R. 2006, Java Man’s first tools. Science, 312(5772): 361. (en anglais) (“les premiers outils de l'Homme de Java”) doi:10.1126/science.312.5772.361

Storm, P., Aziz, F., Vos, J. de, Kosasih, D., Baskoro, S., Ngaliman, \& Van den Hoek Ostende, L. W. 2005. Late Pleistocene Homo sapiens in a tropical rainforest fauna in East Java. Journal of Human Evolution, 49: 536-545. (en anglais) ("Homo sapiens Pléistocène tardif dans une faune de forêt tropicale humide dans l'Est de Java”) doi:10.1016/j.jhevol.2005.06.003

Storm, P., Wood, R., Stringer, C., Bartsiokas, A., De Vos, J., Aubert, M., Kinsley, L., \& Grün, R. 2013, U-series and radiocarbon analyses of human and faunal remains from Wajak, Indonesia. Journal of Human Evolution, 64: 356-365. (en anglais) (“Analyses Uth et radiocarbone de restes humains et fauniques à Wajak, Indonésie”) doi:10.1016/j.jhevol.2012.11.002

Summerhayes, G. R., Leavesley, M., Fairbairn, A., Mandui, H., Field, J., Ford, A., \& Fullagar, R. 2010, Human Adaptation and Plant Use in Highland New Guinea 49,000 to 44,000 Years Ago. Science, 330: 78-81. (en anglais) (“L'adaptation humaine et l'utilisation des plantes dans les régions montagneuses de la Nouvelle-Guinée 49,000 à 44,000 ans”) doi:10.1126/science.1193130

Sutikna, T., Tocheri, M. W., Morwood, M. J., Saptomo, E. W., Jatmiko., Rokus Due Awe., Wasisto, S., Westaway, K. E., Aubert, M., Li, B., Zhao, J. X., Storey, M., Alloway, B. V., Morley, M. W., Meijer, J. M., Van den Bergh, G. D., Grûn, R., Dosseto, A., Brumm, A., Jungers, W. L., \& Roberts, G. 2016, Revisited stratigraphy and chronology for Homo floresiensis at Liang Bua in Indonesia. Nature, 532(7599): 366-369. (en anglais) ("Stratigraphie revisité et chronologie pour Homo floresiensis à Liang Bua en Indonésie”) doi:10.1038/nature17179 
Swisher, C., Rink, J., Anton, S., Schwarcz, H., Curtis, G., Suprijo, A., \& Widiasmoro. 1996. Latest Homo erectus of Java: potential contemporaneity with Homo sapiens in Southeast Asia. Science, 274: 1870-1874. (en anglais) (“Nouvel Homo erectus de Java: contemporanéité potentielle avec Homo sapiens en Asie du Sud-Est”)

Tanudirjo, D.-A. 2005, Long continuous or short occasional occupation? The human use of Leang Sarru rock shelter in the Talaud Islands, North Eastern Indonesia. Bulletin of Indo-Pacific Association Bulletin, 25: 15-19. (en anglais) (“Occupation longue continue ou courte occasionnelle? L'utilisation humaine de l'abri rocheux de Leang Sarru dans les îles Talaud, dans le nord-est de l'Indonésie”) doi:10.7152/bippa.v25i0.11910

Tenazas, R. 1974, A progress report on the Magsuhot excavations in Bacong, Negros Oriental. Philippine Quarterly of Culture and Society (PQCS), 2: 133-155. (en anglais) (“Un rapport d'étape sur les fouilles de Magsuhot à Bacong, Negros Oriental”) URL: http://www.jstor.org/stable/29791135

Tenazas, R. 1985, A note on stone and shell implements from late Palaeolithic and Neolithic sites in Carcar, Cebu, Philippines. In: Recent advances in indo-pacific prehistory. (Misra, V. N., \& Bellwood, P., Eds.), Oxford \& IBH Publishing, Leiden Brill, Leiden: p. 207-209. (en anglais) ("Une note sur les outils en pierre et en coquillages des sites du Paléolithique supérieur et du Néolithique à Carcar, Cebu, Philippines”)

Tiauzon, A. 2011, Lithic technology of Song Terus during the Late Middle Pleistocene to Early Upper Pleistocene. Master's thesis IMQP, Muséum National d'Histoire Naturelle, Paris, 96 p. (en anglais) ("La technologie lithique de Song Terus au cours du Pléistocène moyen supérieur au Pléistocène supérieur précoce”)

Torrence, R. 2004, Pre-Lapita valuables in Island Melanesia. In A Pacific Odyssey: Archaeology and Anthropology in the Western Pacific. Papers in Honour of Jim Specht. Records of the Australian Museum, Supplement 29: 163-172. (en anglais) ("Objets de valeur pré-Lapita dans l'île Mélanésie”) doi:10.3853/j.0812-7387.29.2004.1413

Torrence, R., Kelloway, S., \& White, P. 2013, Stemmed tools, social interaction and voyaging in Early-mid Holocene Papua New Guinea. The Journal of Island and Coastal Archaeology, 8(2): 278-310. (en anglais) (“Outils à pédoncule, interaction sociale et voyage au début et mi-Holocène en Papouasie-Nouvelle-Guinée”) doi:10.1080/15564894.2012.761300

Turney, C. S. M., Birda, M. I., Fifield, L.K., Roberts, R. G., Smith, M., Dortch, C. E., Grün, R., Lawson, E., Ayliffe, L. K., Miller, G. H., Dortch, J., \& Cresswell, R. G. 2001,Early human occupation at Devil's Lair, southwestern Australia 50,000 years ago. Quaternary Research, 55: 3-13. (en anglais) (“Occupation humaine précoce à Devil's Lair, dans le sud-ouest de l'Australie, il y a 50,000 ans”) doi:10.1006/qres.2000.2195

Van den Bergh, G. D., de Vos, J., Sondaar, P. Y., \& Aziz, F. 1996, Pleistocene zoogeographic evolution of Java (Indonesia) and glacio-eustatic sea level fluctuations: a background for the presence of Homo. Bulletin of Indo-Pacific Prehistory Association, 14(1): 7-21. (en anglais) ("Évolution zoogéographique pléistocène de Java (Indonésie) et les fluctuations du niveau de la mer glacio-eustatique: un arrière plan de la présence de l'Homo") doi:10.7152/bippa.v14i0.11583 
Van den Bergh, G. D. 1997, The Late Neogene elephantoid-bearing faunas of Indonesia and their palaeozoogeographic implications: a study of the terrestrial faunal succession of Sulawesi, Flores, and Java, including evidence for early hominid dispersal east of Wallace’s Line. Scripta Geologica, 117: 1- 419. (en anglais) ("Les faunes d'éléphantoïdes du Néogène tardif en Indonésie et leurs implications paléozoogéographiques: une étude de la succession faunique terrestre de Sulawesi, Flores et Java, incluant des preuves de dispersion précoce d'hominidés à l'est de la ligne de Wallace”)

Van den Bergh, G. D., Brumm, A., Puspaningrum, M., Setiabudi, E., Kurniawan, I., \& Prasetyo, U. W. 2014, Taphonomy of Stegodon florensis remains from the early Middle Pleistocene archaeological site Mata Menge, Flores, Indonesia. Abstract Book of the $\mathrm{VI}^{\text {th }}$ International Conference on Mammoths and their Relatives. Scientific Annals of the School of Geology special volume 102, Aristotle University of Thessaloniki, Thessaloniki: p. 207-208. (en anglais) ("Taphonomie des restes de Stegodon florensis sur le site archéologique du Pléistocène moyen Mata Menge, Flores, Indonésie”)

Van den Bergh, G. D., Li, B., Brumm, A., Grün, R., Yurnaldi, D., Moore, M. W., Kurniawan, I., Setiawan, R., Aziz, F., Roberts R. G., Suyono, Storey, M., Setiabudi, E., \& Morwood, M. J. 2016a, Earliest hominid occupation of Sulawesi, Indonesia. Nature, 529(7585): 208-211. (en anglais) ("Première occupation des hominidés de Sulawesi, en Indonésie”) doi:10.1038/nature17999

Van den Bergh, G. D., Kaifu, Y., Kurniawan, I., Kono, R. T., Brumm, A., Setiabudi, E., Aziz, F., \& Morwood, M. J. 2016b, Homo floresiensis-like fossils from the early Middle Pleistocene of Flores. Nature, 534: 245-248. (en anglais) ("Fossiles type Homo floresiensis du début du Pléistocène moyen à Flores”) doi:10.1038/nature16448

Van Heekeren, H. R. 1972, The stone age of Indonesia. (2nd. revised edition) with a contribution by R. P. Soejono. Nijhoff, The Hague, 328 p. (en anglais) ("L'âge de pierre de l'Indonésie”)

Van Heteren, A. H., \& de Vos, J. 2012, Stone implements from Java and Flores: A history of the discoveries. C. R. Palevol, 11(2-3): 181-189. (en anglais) ("Outillage en pierre de Java et Flores: Une histoire des découvertes”) doi:10.1016/j.crpv.2011.07.006

Van Stein Callenfels, P. V. 1938, The Proto-Toalian. Tijdschr. Indische Taal-, Land- en Volkenk. 68: 579-584. (en anglais) (“Le Proto-Toalian”)

Verhoeven, T. 1958, Pleistozäne Funde in Flores. Anthropos, 53: 264-265. (en allemand ("French-Indonesian archaeological excavation in East Kalimantan")

Verhoeven, T. 1968, Vorgeschichtliche Forschungen auf Flores, Timor und Sumba. Anthropica. Anthropos, 21: 393-403. (en allemand ("Recherches préhistoriques sur les îles de Flores, Timor et Sumba”)

Von Koenigswald, G. H. R., \& Gosh, A. K. 1973, Stone implements from the Trinil Beds of Sangiran, Central Java. Proceedings of the Koninklijke Nederlandse Akademie van Wetenschappen, B76: 1-34. (en anglais) ("Outillage en pierre des lits Trinil de Sangiran, Java central”)

Voris, H. K. 2000, Maps of Pleistocene sea levels in southeast Asia: shorelines, river systems and time durations. Journal of Biogeography, 27: 1153-1161. (en anglais) ("Cartes des niveaux du Pléistocène en Asie du Sud-Est: rivages, systèmes fluviaux et durées”) 
Widianto, H. 1997, Ekskavasi Situs Gua Babi, Kabupaten Tabalong, Provinsi Kalimantan Selatan. Berita Penelitian Arkeologi Balai Arkeologi Banjarmasin, no. 1, Banjarmasin. (en indonésien) ("Recherches préhistoriques sur les îles de Flores, Timor et Sumba”)

Widianto, H., Toha, B., \& Simanjuntak, H. T. 2001, The discovery of stone implements in the Grenzbank: new insights into the chronology of the Sangiran Flake industry. IndoPacific Prehistory Association Bulletin, 21: 157-161. (en anglais) ("La découverte des outils en pierre dans le Grenzbank: de nouvelles perspectives dans la chronologie de l'industrie en pierre taillée de Sangiran”) doi:10.7152/bippa.v21i0.11777

Woo, J. 1959, Liukiang Man-earliest representative of modern man in East Asia. Science Record, new series, 3: 165-67. (en anglais) (“Liukiang Man-premier représentant de l'homme moderne en Asie de l'Est”)

Wu, R. 1983, Hominid fossils from China and their bearing on human evolution. Canadian Journal of Anthropology, 3: 207-14. (en anglais) ("Fossiles d'hominidés de Chine et leur incidence sur l'évolution humaine”)

Xu, C., Zhang, Y., Chen, C., \& Fang, D. 1984, Human occipital bone and mammalian fossils from Chaoxian, Anhui. Acta Anthropologica Sinica, 3: 202-9. (en anglais) (“Ossements occipitaux humains et des fossiles de mammifères de Chaoxian, Anhui”)

Yokoyama, Y., Falguères, C., Sémah, F., Jacob, T., \& Grün, R. 2008. Gamma-Ray spectrometric dating of late Homo erectus skulls from Ngandong and Sambungmacan, Central Java, Indonesia. Journal of Human Evolution, 55: 274-77. (en anglais) ("Datation par spectrométrie gamma des crânes tardifs Homo erectus de Ngandong et Sambungmacan, Java central, en Indonésie”)

Zeitoun, V., Détroit, F., Grimaud-Hervé, D. \& Widianto, H. 2010, Solo man in question: convergent views to split Indonesian Homo erectus in two categories. Quaternary International, 223-224: 281-292. (en anglais) ("Homme solo en question: des vues convergentes pour diviser l'Homo erectus indonésien en deux catégories”) doi:10.1016/j.quaint.2010.01.018

Zeitoun, V., Barriel, V. \& Widianto, H. 2016, Phylogenetic analysis of the calvaria of Homo floresiensis. Comptes Rendus Palevol, 15(5): 555-568. (en anglais) (“Analyse phylogénétique de la calvaria de Homo floresiensis”) doi:10.1016/j.crpv.2015.12.002

Zhou, M., Li, Y., \& Wang, L. 1982, Chronology of the Chinese fossil hominids. In: L'Homo erectus et la place de l'homme de Tautavel parmi les hominidés fossiles. (de Lumley, H., Ed.), $1^{\text {er }}$ congrès international de Paléontologie humaine, Nice, 16-21 octobre 1982. Institut de Paléontologie Humaine, Paris: p. 593-604. (en anglais) (“Chronologie des hominidés fossiles de Chine par spectrométrie gamma des crânes tardifs Homo erectus de Ngandong et Sambungmacan, Java central, en Indonésie”) 


\title{
The lithic productions of Indonesian Archipelago
}

\author{
Hubert Forestier ${ }^{1}$, Michel Grenet ${ }^{2}$, Antony Borel ${ }^{3}$, Vincenzo Celiberti ${ }^{4}$
}

1. Muséum national d’Histoire naturelle, UMR 7194 CNRS-MNHN-UPVD, Institut de Paléontologie Humaine, 1 rue René-Panhard, 75013 Paris, France. Email: hubforestier@gmail.com

2. UMR 5608 Traces, maison de la recherche, Université de Toulouse II - le Mirail, 5 allée Antonio Machado, FR31508 Toulouse, France. Email: archelmi@gmail.com

3. Muséum national d’Histoire naturelle, UMR 7194 CNRS-MNHN-UPVD, Musée de l’Homme, 17 place du Trocadéro, 75116 Paris, France. Email: borel@mnhn.fr

4. Université de Perpignan Via Domitia, UMR 7194 CNRS-MNHN-UPVD, Centre Européen de Recherches

Préhistoriques de Tautavel, Avenue Léon-Jean Grégory, 66720 Tautavel, France.

Email: vincenzo.celiberti@cerptautavel.com

\begin{abstract}
:
Drawing up a synthesis on the prehistory of an archipelago and its lithic assemblages is not an easy thing because of the vastness of the island space with a total surface of two millions $\mathrm{km}^{2}$, and because the notion of Palaeolithic is inapplicable, especially the "Upper Palaeolithic" or "Epipalaeolithic-Mesolithic" used for Western Eurasia. Indonesia and its myriad of islands and islets (about 18,000) are part of a 5,000 km by 2,000 km rectangle on both sides of the equator, making it the largest archipelago in the world. This immense geographical area stretching over a maritime area of about 6 million $\mathrm{km}^{2}$, does not allow us today to treat exhaustively all industrial groups, facies or prehistoric cultures. For this reason we will only discuss the main ones. Indonesia occupies a privileged place for the history of fossil men, which makes it unavoidable in the knowledge of the variability of the behavior of hominids in inter-tropical context. The aim of this is to present a critical and objective overview on the various known methods for knapping stone for the last 1 million year on the main islands and in the sites with well established and well dated stratigraphy.

Few technocomplexes are well defined before the Holocene, a period when the insularity of this geographical area was set by rising sea level which progressively marked the beginning of the history of the insulindian archipelagos. These include the "Toalian" in Sulawesi (facies with points), the "Sampungian" (facies with points) and Song Keplek or "Keplekian" flake industries (orthogonal débitage) in the eastern part of the Java Island. Other than these three technical traditions which remain recognizable on a typo-technological level, most of the other materials encountered do not correspond to distinct facies associated with specific tools. As a general rule, the Upper Pleistocene and Indonesian Holocene lithic industries respond to a basic production of flakes and flake tools with multiple regional variants. The débitage mode is very largely made with hard stone direct percussion, non Levallois, rarely Discoid, and with no blade-bladelet productions as seen in Western Europe or in the Near and Middle East. Unlike continental South-East Asia, where it is even more difficult to discern an Early, Middle and Upper Palaeolithic due to the continuity of industries all made on pebble (Hoabinhian and others), Indonesia (which becomes insular during the Pleistocene-Holocene transition) presents an unprecedented heterogeneity of lithic assemblages. That is to say, a diversity in the lithic production methods according to different
\end{abstract}


chaînes opératoires for shaping (pebble, Sampung type point, biface, etc.) or for débitage (discoid, orthogonal and laminar).

The purpose of this article is to summarize the main lithic assemblages of the Indonesian Archipelago based on a selection of islands on which lithic assemblages have been properly documented. Our focus will be on the islands of Sumatra, Java, Kalimantan (Borneo), Sulawesi, and other more oriental ones, which, like Timor and Flores, have in recent years provided major discoveries in Palaeoanthropology and in Prehistory. We have been forced to make regional and chronological divisions (Early-Middle Pleistocene and Late Final Pleistocene - Early Holocene) in order to better present this review on lithic assemblages that have not all been the subject of in-depth technological studies, especially from the chaîne opératoire concept point of view.

Keywords: Indonesia; Insular Southeast Asia; Discoidal; orthogonal debitage; laminar debitage; bifacial point 